\title{
Avaliação e comparação de desempenho entre a arquitetura IP e a arquitetura IP sobre MPLS
}

Carlos Marcos Kakihara

Dissertação apresentada ao Instituto de Física de São Carlos, Universidade de São Paulo, para obtenção do título de Mestre em Ciências: Física Aplicada - opção Física Computacional.

Orientador: Prof. Dr. Carlos Antônio Ruggiero

SÃO CARlos

2006 
Kakihara, Carlos Marcos

Avaliação e comparação de desempenho entre a arquitetura IP e a arquitetura IP sobre MPLS/Carlos Marcos Kakihara. - São Carlos, 2006.

$103 p$.

Dissertação (Mestrado) - Instituto de Física de São Carlos, 2006.

Orientador: Prof. Dr. Carlos Antônio Ruggiero

1. MPLS. 2. Label switching. 3. Engenharia de tráfego.

I. Título. 
aos meus pais,

por me dar amor, por me dar segurança, pelo exemplo de caráter e ternura, para que hoje eu tenha a consciência de que serei sempre grato pelo que tenho de mais valioso: a minha formação;

aos meus irmãos, por me ensinarem que tenho muito o que aprender na vida,

e por poder contar com eles sempre;

à minha noiva, por iluminar o meu caminho nos momentos mais difíceis,

e por me inspirar a viver com amor e alegria. 


\section{Agradecimentos}

- Ao Prof. Dr. Carlos Antônio Ruggiero, por depositar em mim sua confiança, e me honrar com uma fração dos seus conhecimentos acadêmicos e pessoais.

- À PRODESP, em especial à Fábrica de Software, pelo incentivo à pesquisa nas áreas da Computação.

- Aos meus amigos, que entenderam a minha ausência e me apoiaram durante todo o mestrado, e estarão sempre de braços abertos para me receber de volta. 


\section{Sumário}

1 Introdução 1

2 IP: Conceitos fundamentais $\quad 3$

2.1 Endereçamento . . . . . . . . . . . . . . . 7

2.2 Cabeçalho ........................... 13

2.3 Roteamento . . . . . . . . . . . . . . . 18

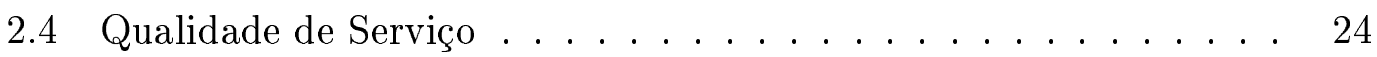

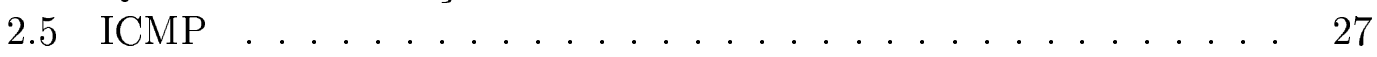

3 MPLS: Conceitos fundamentais $\quad 29$

3.1 Elementos de uma rede MPLS . . . . . . . . . . . . . 31

3.2 Criação e manutenção de LSPs . . . . . . . . . . . . . 34

3.3 Cabeçalho ....................... 36

3.4 Fragmentação . . . . . . . . . . . . . . . . . . . . . . . . . . . . . . . .

3.5 Qualidade de Serviço . . . . . . . . . . . . . . 41

4 Breve comparação entre redes IP e redes IP sobre MPLS 43

4.1 Modelo OSI . . . . . . . . . . . . . . . . . . 43

4.2 Formato de cabeçalho. . . . . . . . . . . . . 44

4.3 Endereçamento . . . . . . . . . . . . . . . . 44

4.4 Encaminhamento x Roteamento . . . . . . . . . . . . . 44

4.5 Rotas pré-determinadas ...................... 46

4.6 Relevância do ponto de entrada . . . . . . . . . . . . . . . 46

4.7 Escalabilidade . . . . . . . . . . . . . . . . 47

4.8 Engenharia de Tráfego e Qualidade de Serviço . . . . . . . . . . 47

5 Análise de desempenho $\quad 49$

5.1 Avaliação inicial dos roteadores . . . . . . . . . . . . . . 51

5.2 Processamento de cabeçalho e encapsulamento . . . . . . . . . . . . 54

5.3 Quantidade de nós intermediários . . . . . . . . . . . . 64

5.4 Engenharia de tráfego . . . . . . . . . . . . 76

6 Conclusões $\quad 91$

6.1 Desempenho .......................... 91

6.2 Otimização de recursos . . . . . . . . . . . . . . . . 99 93

6.3 Implementação: Projeto KyaTera . . . . . . . . . . . . . . . 95

6.4 Considerações finais . . . . . . . . . . . . . . . 97 
7 Trabalhos futuros 


\section{Lista de Figuras}

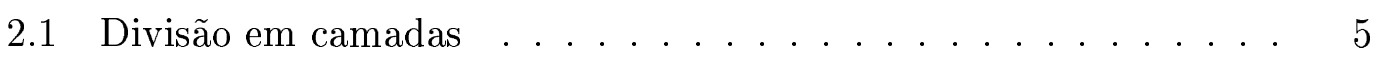

2.2 Roteamento independente . . . . . . . . . . . 7

2.3 Divisão em netid e hostid . . . . . . . . . . . . . . . 10

2.4 Divisão em sub-redes . . . . . . . . . . . . . . . . . . 12

2.5 Datagrama IP . . . . . . . . . . . . . . . . . . . . . 14

2.6 TOS com os bits D, T e R . . . . . . . . . . . 14

2.7 TOS unificado............................ 15

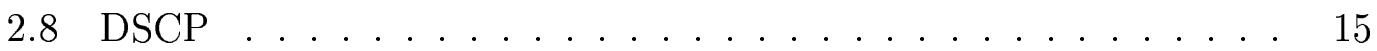

2.9 Duas opções de ligação entre as redes B e C . . . . . . . . . . 19

2.10 Falha no roteador R3: R4 passa a ligar as redes B e C . . . . . 22

3.1 Componentes de uma rede MPLS . . . . . . . . . . . 34

3.2 Cabeçalho MPLS . . . . . . . . . . . . . 36

3.3 Cabeçalho MPLS em diferentes protocolos da camada $2 \ldots 39$

3.4 Hierarquia: 2 níveis de profundidade . . . . . . . . . 39

5.1 Avaliação individual dos roteadores . . . . . . . . . . . . 51

5.2 Capacidade individual de roteamento IP . . . . . . . . . . . 54

5.3 Dois roteadores IP . . . . . . . . . . . . . . . . . 54

5.4 Dois roteadores MPLS . . . . . . . . . . . . . . 58

5.5 Desempenho dos roteadores . . . . . . . . . . . 64

5.6 Roteamento IP com três roteadores . . . . . . . . . . . . 65

5.7 Três LSRs . . . . . . . . . . . . . . . . . . 67

5.8 Roteamento com roteadores em posições inadequadas . . . . . . . 73

5.9 Roteamento em posições adequadas . . . . . . . . . . . . . . 75

5.10 Quatro nós MPLS . . . . . . . . . . . . . . 75

5.11 Quatro LSRs . . . . . . . . . . . . . . 76

5.12 Rede "peixe" . . . . . . . . . . . . . . . . . . . . . 77

5.13 Roteamento IP: congestionamento na rota A . . . . . . . . . . 78

5.14 Roteamento IP: congestionamento na rota B . . . . . . . . . 80

5.15 Roteamento IP/MPLS . . . . . . . . . . . . . . . . 82

5.16 Gráfico comparativo na "rede peixe" . . . . . . . . . . 89

5.17 Roteamento na rede "peixe" otimizada . . . . . . . . . . . . 90

6.1 Desempenho com dois roteadores . . . . . . . . . . . . . 92

6.2 Posicionamento inadequado dos LSRs . . . . . . . . . . . . . . . . 92

6.3 Posicionamento ideal dos LSRs . . . . . . . . . . . . . . . . . 93

6.4 Segregação de tráfego . . . . . . . . . . . . . . . 94 
6.5 Protótipo para o KyaTera ................. 96 


\section{Lista de Tabelas}

2.1 Tabela de rotas de R1 utilizando endereços individuais . . . . . 20

2.2 Tabela de rotas de R1 utilizando endereços de rede . . . . . . 20

2.3 Tabela de rotas de R1 utilizando uma rota padrão . . . . . . . . . 21

2.4 Tabela incorreta de rotas em R1 . . . . . . . . . . . . . 21

2.5 AF: 12 possíveis classes de serviço . . . . . . . . . . 27

5.1 Descrição dos equipamentos utilizados . . . . . . . . . 50

5.2 Roteamento IP com dois nós intermediários sem MPLS . . . . . 56

5.3 Roteamento IP com dois nós intermediários e com MPLS . . . . . 58

5.4 Capacidade de roteamento com dois nós intermediários . . . . . 63

5.5 Roteamento IP com três LSRs sem MPLS . . . . . . . . . . . 66

5.6 Roteamento IP sobre MPLS com três LSRs . . . . . . . . . . 73

5.7 Roteamento IP na rede "peixe" pela rota A . . . . . . . . . . . 79

5.8 Roteamento IP na rede "peixe" pela rota B . . . . . . . . . . . 81

5.9 Roteamento IP sobre MPLS na rede "peixe" . . . . . . . . . . 89 


\section{Acrônimos}

AF Assured Forwarding

ATM Asynchronous Transfer Mode

BGP Border Gateway Protocol

CPU Central Processing Unit

DARPA Defense Advanced Research Projects Agency

DF Do not fragment

DS Differentiated Services

DSCP DS Codepoint

ECN Explicit Congestion Notification

EESC Escola de Engenharia de São Carlos

EF Expedited Forwarding

EXP Experimetal

DC-UFSCAR Departamento de Computação - UFSCAR

DSCP Differentiated Services Code Point

FEC Forwarding Equivalence Classes

FTP File Transfer Protocol

GPL General Public License

ICMP Internet Control Message Protocol

ICMC Instituto de Ciências Matemáticas e Computação

IETF Internet Engineering Task Force

IFSC Instituto de Física de São Carlos

ILM Incoming Label Map

IP Internet Protocol 
IPv4 Internet Protocol version 4

IPv6 Internet Protocol version 6

LDP Label Distribuition Protocol

LER Label Edge Router

LIFO Last-In-First-Out

LSP Label Switched Path

LSR Label Switching Router

MIT Massachusetts Institute of Technology

MF More Fragments

MPLS MultiProtocol Label Switching

MTU Maximum Transfer Unit

NAT Network Address Translation

NHLFE Next Hop Label Forwarding Entry

OSI Open Systems Interconnection

OSPF Open Shortest Path First

PCI Peripheral Component Interconnect

PHB Per Hop Behavior

POS Packet Over SONET

PPP Point-to-Point Protocol

QoS Quality of Service

RSVP Resource Reservation Protocol

SEL-EESC Departamento de Engenharia Elétrica - EESC

SMTP Simple Mail Transfer Protocol

SSH Secure Shell

TCP Transmission Control Protocol

TOS Type of Service

TTL Time to Live

UC University of California

UCLA University of California - Los Angeles 
UDP User Datagram Protocol

UFSCAR Universidade Federal de São Carlos

USP Universidade de São Paulo

VCI Virtual Channel Identifier

VPI Virtual Path Identifier

VPN Virtual Private Network 


\section{Resumo}

Este trabalho apresenta uma comparação e avaliação das arquiteturas de roteamento IP e IP sobre MPLS. Inicialmente, são apresentados alguns conceitos básicos dos protocolos IP e MPLS, e suas relações com a camada 2 do modelo OSI. Duas características foram analisadas: o processo de encaminhamento e as granularidades para engenharia de tráfego. A medição de desempenho foi feita com computadores Linux realizando a tarefa de roteamento/encaminhamento de datagramas IP em diferentes tipos de redes Ethernet. Com base nos resultados dos testes, é feita uma discussão sobre o desempenho e topologias adequadas para cada arquitetura. 


\section{Abstract}

This work presents a comparison and evaluation of IP and the IP over MPLS routing architectures. Initially, some basic concepts of IP and MPLS protocols and their relationship with layer 2 of OSI model are introduced. Two characteristics were analyzed: the forwarding process and traffic engineering granularity. The performance analysis was carried out on Linux computers routing/forwarding IP datagramas at different types of Ethernet networks. Considering the results of the tests, a discussion about performance and suitable topologies is made for each architecture. 


\section{Capítulo 1}

\section{Introdução}

Para atender às demandas causadas pelo aumento da utilização da Internet, novas tecnologias estão sendo constantemente desenvolvidas. No entanto, os esforços não se concentram apenas no aumento da capacidade dos equipamentos. A utilização mais racional dos recursos tem um efeito considerável na qualidade do tráfego na Internet.

Recentemente, uma nova tecnologia vem ganhando força como alternativa à clássica combinação protocolo Internet Protocol (IP) sobre camada 2 (Ethernet, Asynchronous Transfer Mode (ATM), Packet Over SONET (POS) e Frame Relay): a arquitetura IP sobre MultiProtocol Label Switching (MPLS). Combinando roteamento de nível 3 com chaveamento de nível 2, essa nova arquitetura oferece maior possibilidade de gerenciamento e engenharia de tráfego, além de reduzir o processamento necessário para realizar o roteamento de datagramas de rede.

Este trabalho consiste em uma análise comparativa entre roteamento IP (versão 4) tradicional e roteamento IP sobre MPLS em redes Ethernet. Neste documento, a expressão "roteamento IP" representa o roteamento em arquiteturas IP diretamente sobre Ethernet, e a expressão "roteamento IP sobre MPLS" ou "arquitetura IP sobre MPLS" representa a combinação de roteamento IP com encaminhamento através do protocolo MPLS em redes Ethernet.

Os fatores avaliados foram a diferença de desempenho no processo de rotea- 
mento e as possibilidades oferecidas na diferenciação de tráfego.

Os capítulos 2 e 3 apresentam os conceitos básicos sobre os protocolos IP e MPLS, respectivamente. Serão descritos as estruturas de dados, os conceitos e os mecanismos de endereçamento e roteamento das duas arquiteturas.

O capítulo 4 aponta algumas diferenças entre as duas arquiteturas e as vantagens e desvantagens de cada uma delas.

O capítulo 5 descreve os experimentos realizados, a metodologia utilizada e traz uma discussão dos resultados obtidos. Foram construídos quatro ambientes de rede, com diferentes topologias para avaliar o comportamento das duas arquiteturas. Os dois principais focos de estudo foram a diferença de desempenho devido ao formato dos cabeçalhos e as possibilidades de otimização dos recursos de rede oferecidas pelas duas arquiteturas.

O capítulo 6 traz as conclusões elaboradas com base no estudo da teoria e nos resultados práticos. Neste capítulo também são apresentadas as recomendações de uso das duas arquiteturas em diferentes casos de topologia de rede.

O capítulo 7 relaciona alguns estudos interessantes a serem realizados e que poderiam complementar este trabalho. 


\section{Capítulo 2}

\section{IP: Conceitos fundamentais}

A primeira documentação registrada sobre a rede que se tornaria a Internet de hoje foi feita por J.C.R. Licklider do Massachusetts Institute of Technology (MIT) em agosto de 1962, discutindo seu conceito de "rede galáctica" [1]. Nesse mesmo ano, Licklider iniciou e liderou o programa de pesquisa no Defense Advanced Research Projects Agency (DARPA), enfatizando a importância de uma rede global.

Paralelamente, Leonard Kleinrock do MIT publicou, em julho de 1961, o primeiro artigo sobre o conceito de redes de comutação de pacotes [2]. A idéia foi adotada por Lawrence G. Roberts, sucessor de Licklider no DARPA, que em 1965 criou a primeira rede de longa distância, conectando um computador no MIT a outro na Califórnia. Com o sucesso dessa conexão, o DARPA iniciou o desenvolvimento da ARPANET e em 1969, contava com quatro computadores interligados: University of California - Los Angeles, Stanford Research Institute, University of California - Santa Barbara e University of Utah.

Os protocolos evoluíram e no final da década de 70 chegaram ao que são hoje: a arquitetura $T C P / I P$. Ela é formada por um conjunto de protocolos, tais como IP, Transmission Control Protocol (TCP), User Datagram Protocol (UDP), Internet Control Message Protocol (ICMP), Simple Mail Transfer Protocol (SMTP), File Transfer Protocol (FTP) entre outros, mas é conhecida como TCP/IP devido à 
importância dos protocolos IP e TCP em relação aos demais.

Assim como o modelo Open Systems Interconnection (OSI) [3], a arquitetura TCP/IP possui uma divisão em camadas (figura 2.1).

No modelo OSI, existem 7 camadas, e os princípios para a criação das mesmas foram os seguintes:

- Uma camada para cada abstração, realizando uma função bem definida;

- Os limites entre as camadas devem contribuir para minimizar a troca de informações entre elas;

- A quantidade de camadas deve ser suficiente para possibilitar a distribuição das diferentes funções e, ao mesmo tempo, não tornar a arquitetura sobrecarregada, com muitas camadas definidas.

O modelo OSI foi criado antes dos protocolos que o próprio OSI adotaria (X25 [4], por exemplo). Isso o torna um modelo amplo e genérico, portanto, muito útil conceitualmente. Em contrapartida, a sua implementação é muito complexa, dificultando a sua utilização nos sistemas operacionais e softwares de rede.

Já o modelo TCP/IP possui apenas 4 camadas: Host-to-network, Internet, Transport e Application. As camadas 5 e 6 do modelo OSI não existem no modelo TCP/IP, e as camadas 1 e 2 do modelo OSI aparecem unificadas na camada 1 do modelo TCP/IP.

O modelo TCP/IP foi criado depois dos protocolos, como se fosse uma descrição dos protocolos já existentes. Isso traz conseqüências favoráveis e desfavoráveis ao modelo. Como conseqüência favorável, podemos citar a menor complexidade do modelo TCP/IP em relação ao modelo OSI, o que facilita a sua implementação em sistemas operacionais e softwares de rede. Além disso, temos o encaixe perfeito entre modelo e protocolos. Como desvantagem, podemos citar o fato de que o modelo TCP/IP não serve para descrever outra pilha de protocolos que não seja a pilha TCP/IP. 
A divisão em camadas oferece duas grandes vantagens. A primeira é a possibilidade de um desenvolvimento independente de cada camada. Por exemplo, para se desenvolver um novo sistema de roteamento, não é necessário ter conhecimento aprofundado das tecnologias da camada 2, assim como os protocolos da camada 4 (TCP, por exemplo) não precisam se preocupar em descobrir o melhor caminho até o seu destino.

\begin{tabular}{|c|c|}
\hline os & SI \\
\hline$\underline{7 \text { - APPLICATION }}$ & \\
\hline$\underline{6-P R E S E N T A T I O N}$ & \\
\hline 5 - SESSION & \\
\hline$\underline{4 \text { - TRANSPORT }}$ & \\
\hline$\underline{3}$ - NETWORK & \\
\hline$\underline{2}$ - DATA LINK & \\
\hline$\underline{1-P H Y S I C A L}$ & \\
\hline
\end{tabular}

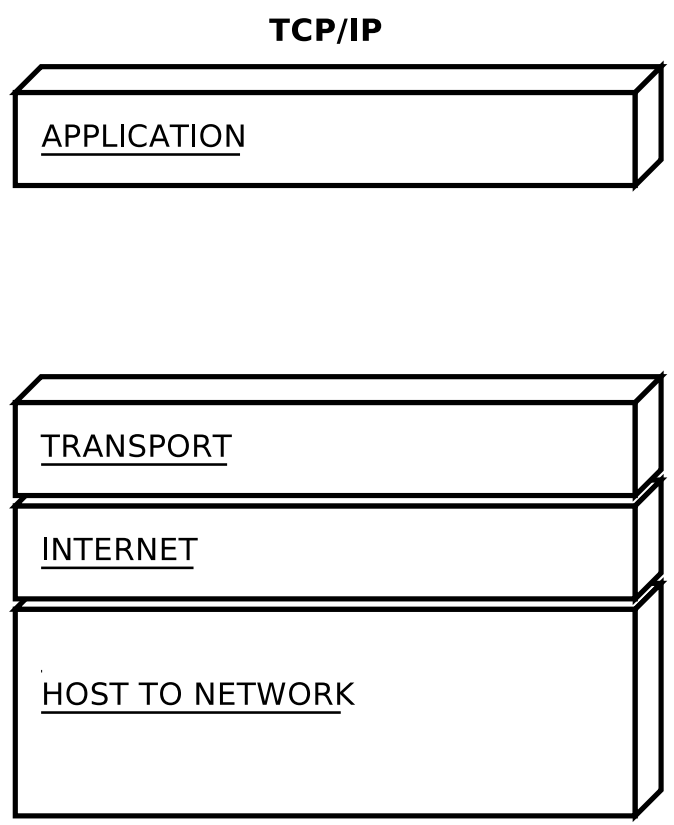

Figura 2.1: Divisão em camadas

A outra vantagem da divisão em camadas é a independência de hardware. $\mathrm{O}$ IP é um protocolo de rede, isto é, está no nível 3 da camada OSI, e o controle e funcionamento dos dispositivos estão implementados na camada 2, poupando o IP dessa tarefa.

O IP é implementado como um protocolo sem conexão: cada unidade de transporte (conhecido como pacote ou datagrama IP) trafega independentemente na rede, e cada roteador decide por qual interface deve enviar o datagrama, seguindo tabelas internas que são atualizadas periodicamente.

O tráfego de informações em uma rede IP pode ser comparado a um sistema de vagões motorizados sobre uma malha de trilhos em uma fábrica de motores. Os vagões representam um datagrama IP. Cada vagão tem um limite de peso que 
pode ser transportado em uma viagem, e quando o peso de um motor ultrapassa o limite máximo, ele precisa ser desmontado e as peças distribuídas em diferentes vagões, de maneira que nenhum deles transporte mais peso do que o permitido (não estamos considerando a fragmentação que ocorre devido a restrições dos protocolos da camada 2 [5] e [6]). Além do peso máximo, cada vagão possui duas placas, uma delas contendo o destino da carga e a outra contendo a origem. A função dos vagões é de apenas transportar as peças, não importando quais são elas, e eles seguem independentemente pelos trilhos em direção ao destino.

Os trilhos por onde passam os vagões representam o meio de comunicação entre os nós. Os operadores de trilhos nas encruzilhadas representam os roteadores. Assim que um vagão chega em uma encruzilhada, o operador precisa decidir para qual trilho enviar o vagão, com base na placa de destino da carga. Como a informação que o operador tem é o endereço destino, ele precisa de um mapa dos trilhos para saber para onde desviar o vagão.

Os vagões podem seguir por caminhos diferentes, chegar fora de ordem ou mesmo ter problemas durante o percurso e não alcançar seu destino. Nesses casos, cabe aos montadores receber as peças, verificar se todas chegaram, organizá-las e montar o motor novamente. Podemos perceber claramente que esse processo de montagem é indepentedente do transporte das peças.

Fazendo a analogia, o protocolo IP determina uma quantidade máxima de dados que pode ser transportado em um datagrama. Quando a informação ultrapassa esse limite, ela deve ser divida em diferentes datagramas, de maneira que todos eles sejam menores ou iguais ao tamanho máximo.

Depois de criados, os datagramas IP seguem pela rede local até o próximo nó roteador. Com base nas informações do cabeçalho IP, o roteador decide para onde encaminhar o datagrama.

Os datagramas podem seguir por caminhos diferentes, chegar fora de ordem e até mesmo ser perdidos. Cabe às camadas superiores (transporte ou aplicação) a organização dos datagramas recebidos, bem como fazer um pedido de retrans- 
missão de um datagrama perdido. A função do protocolo IP limita-se apenas à criação e encaminhamento de datagramas. A figura 2.2 ilustra a trajetória de datagramas IP que transportam dados de uma aplicação.

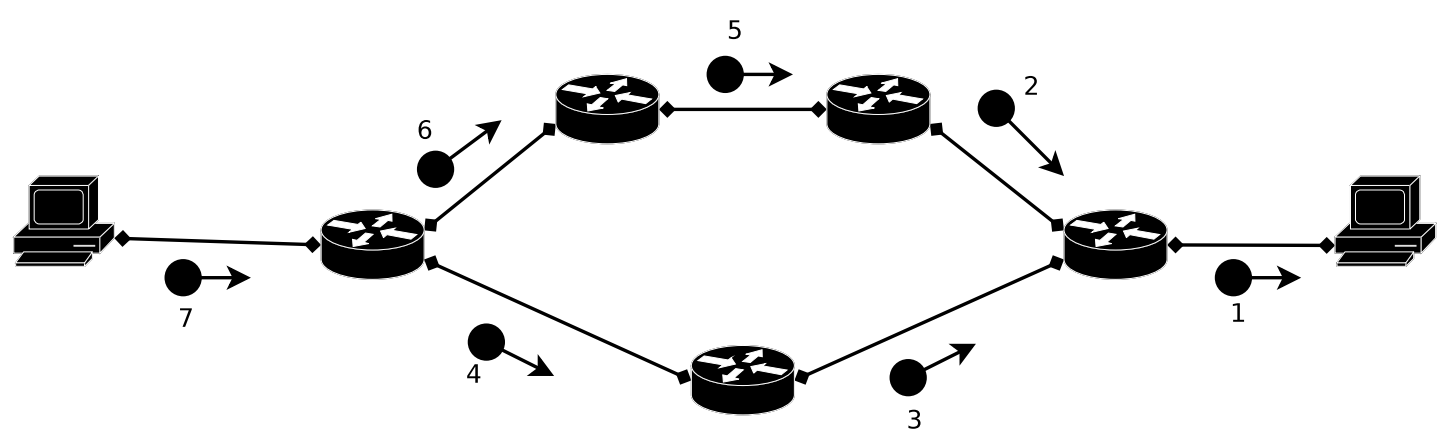

Figura 2.2: Roteamento independente

O primeiro datagrama (1) já está alcançando seu destino (não é possível saber qual rota ele seguiu); o segundo (2) seguiu pela rota mais extensa (quatro roteadores) e está a um roteador do seu destino; o terceiro seguiu pela rota mais curta (três roteadores), e assim por diante. A rota a ser seguida depende de como o primeiro roteador decide encaminhar o datagrama. Através de protocolos de roteamento, os roteadores trocam informações entre si para a definição da melhor rota entre dois pontos.

Quando determinada aplicação exige a confiabilidade de uma conexão em redes IP, é necessário utilizar o TCP, que é um protocolo da camada 4 e oferece essa confiabilidade. Ele se utiliza do IP para transportar os dados em datagramas, e garante a chegada desses dados, íntegros e ordenados, da mesma maneira em que foram enviados. O IP não oferece essa confiabilidade.

\subsection{Endereçamento}

Na Internet, os equipamentos são identificados por números de 32 bits conhecidos como endereços IP. Eles são únicos, ou seja, não pode haver dois ou mais equipamentos utilizando, simultaneamente, o mesmo endereço IP na Internet. 
As máquinas trabalham com as informações no formato binário, assim, um exemplo de endereço IP seria o seguinte:

$$
10001111011010111011010010110001
$$

Para facilitar a interpretação pelas pessoas, os endereços IP são apresentados no formato decimal com pontos:

$$
\text { 143. } 107 . \quad 180 . \quad 177
$$

O endereço IP não representa apenas a identificação do equipamento (interface) em si. Ele é dividido em duas partes: a primeira, conhecida como netid, representa o endereço da rede à qual pertence esse equipamento, e a segunda, conhecida como hostid, representa a sua identificação nessa rede. Nesse endereçamento (conhecido como classful addressing scheme), cada endereço é autoidentificado, isto é, pode-se determinar quais bits correspondem ao netid e quais correspondem ao hostid, e para isso, basta verificar o valor dos três primeiros bits. Estes bits definem também, a qual classe um endereço pertence. No esquema original de endereçamento IP, existem cinco classes de endereços (figura 2.3):

- Classe A: o primeiro bit (mais significativo) é zero e os próximos 7 representam a identificação de rede, e os 24 restantes representam o equipamento na rede. Na notação decimal, o menor endereço é 1.0.0.0, e o maior é 127.255.255.255, o que leva a um número pequeno de redes possíveis. Essa classe é apropriada para redes com um número muito grande de equipamentos, pois pode representar mais de 16 milhões de endereços IP.

- Classe B: o primeiro bit é 1 e o segundo é zero, os próximos 14 bits representam a identificação de rede e os 16 restantes representam o equipamento na rede. Nesta classe, o menor endereço IP é 128.0.0.0 e o maior é 191.255.255.255. O número de equipamentos que podem ser endereçados é relativamente grande: mais de 65 mil. 
- Classe C: o primeiro e o segundo bits são 1, e o terceiro é zero. A identificação de rede é representada pelos próximos 21 bits, o que possibilita a representação de um grande número de redes, sendo o menor endereço é 192.0.0.0 e o maior é 223.255.255.255. Para a representação de equipamentos, são utilizados os últimos 8 bits, que limita em 254 o número máximo de endereços IP.

- Classe D: os três primeiros bits valem 1 e o quarto vale zero. Essa classe é utilizada para identificar grupos de endereços, conhecidos como multicast. O endereçamento varia de 224.0.0.0 a 239.255.255.255, o que possibilita a criação de mais de 200 milhões de grupos distintos. Diferentemente das três classes anteriores, a classe D não possui uma divisão entre netid e hostid: os quatro primeiros bits são fixos e os 28 restantes representam um grupo.

- Classe E: os quatro primeiros bits valem 1. Esta é uma classe de endereços reservada para uso futuro.

Alguns endereços têm significados especiais [7]:

- Rede 127: essa rede classe A funciona como loopback, para testes do protocolo TCP/IP e para comunicações inter-processos na própria máquina. Datagramas com esse prefixo no endereço não devem aparecer em nenhuma rede;

- Endereço com todos os bits em 0: referencia o próprio host. Este caso é utilizado temporariamente quando o host ainda não conhece o netid e o hostid;

- Endereços com todos os bits do netid em 0: referencia o host na rede em que está conectado. Este caso é utilizado temporariamente quando o host ainda não conhece a rede em que está conectado (netid);

- Redes com todos os bits em 1: broadcast na rede local; 


\begin{tabular}{|c|c|c|}
\hline NETID & HOSTID & CLASSE A \\
\hline 00001010 & $00011100 \quad 00011100 \quad 00000001$ & \\
\hline
\end{tabular}

\begin{tabular}{|c|c|}
\hline NETID & HOSTID \\
\hline $10101100 \quad 00011100$ & $00011100 \quad 00000001$ \\
\hline
\end{tabular}

\begin{tabular}{|l|l|}
\hline 110000001010100000011100 & $\underline{00000001}$ \\
\hline
\end{tabular}

CLASSID GROUPID

CLASSE D

$1110 \quad 0000000111000001110000011100$

CLASSID

CLASSE E

1111

Figura 2.3: Divisão em netid e hostid

- Redes com todos os bits do hostid em 1: broadcast direcionado para a rede representada pelo netid;

- Existem vários endereços de grupos multicast reservados: 224.0.0.0 (endereço base), 224.0.0.1 (todos os sistemas de uma sub-rede), 224.0.0.2 (todos os roteadores de uma sub-rede) entre outros.

Esse sistema de endereçamento tem uma limitação importante: cada rede física precisaria de uma rede completa, ou seja, ocuparia no mínimo 254 endereços IP, no caso de uma rede classe C. Isso contribui para um sério problema que surgiu com o crescimento da utilização da Internet: a escassez de endereços IP (este trabalho não aborda Internet Protocol version 6 (IPv6) [8], que oferece um número maior de endereços).

Era muito improvável que os pesquisadores da DARPA imaginassem a velocidade de crescimento da Internet. O número de computadores conectados a essa nova rede pulou de, aproximadamente 200 no início da década de 80, para mais 
de 390 milhões em 2006 [9].

Para combater essa escassez, existem dois mecanismos importantes: máscaras de sub-redes e endereços IP privados.

A máscara de sub-redes permite a divisão de uma rede em blocos menores, através da variação do limite entre a identificação da rede e a identificação do host. Através desse mecanismo é possível se atribuir sub-redes IP a uma determinada rede física, ao invés de se atribuir toda uma rede IP.

Assim, tem-se:

$172.28 .1 .0 / 24$ ou $172.28 .1 .0 / 255.255 .255 .0$

\section{0}

Por exemplo, pelo sistema original de endereçamento, endereços que começam com 172 (octeto mais significativo) pertencem a redes classe B, e utilizariam apenas os dois octetos mais significativos como identificação de rede (172.28). Utilizando-se máscaras de sub-redes, é possível determinar que sejam considerados os três octetos mais significativos como identificação de rede, possibilitando uma distribuição mais adequada para a rede local (figura 2.4).

Assim como é possível dividir uma rede em sub-redes, faixas de redes podem ser representadas por um único par netid e máscara.

192.168.28.0/23 ou 192.168.28.0/255.255.254.0

11000000101010000001110000000000

Redes cujo primeiro octeto vale 192 pertencem à classe $\mathrm{C}$, e utilizam os três primeiros octetos (24 bits) como identificação de rede. Mas, na rede representada por 192.168.28.0/23, são utilizados apenas 23 bits para a sua identificação, 


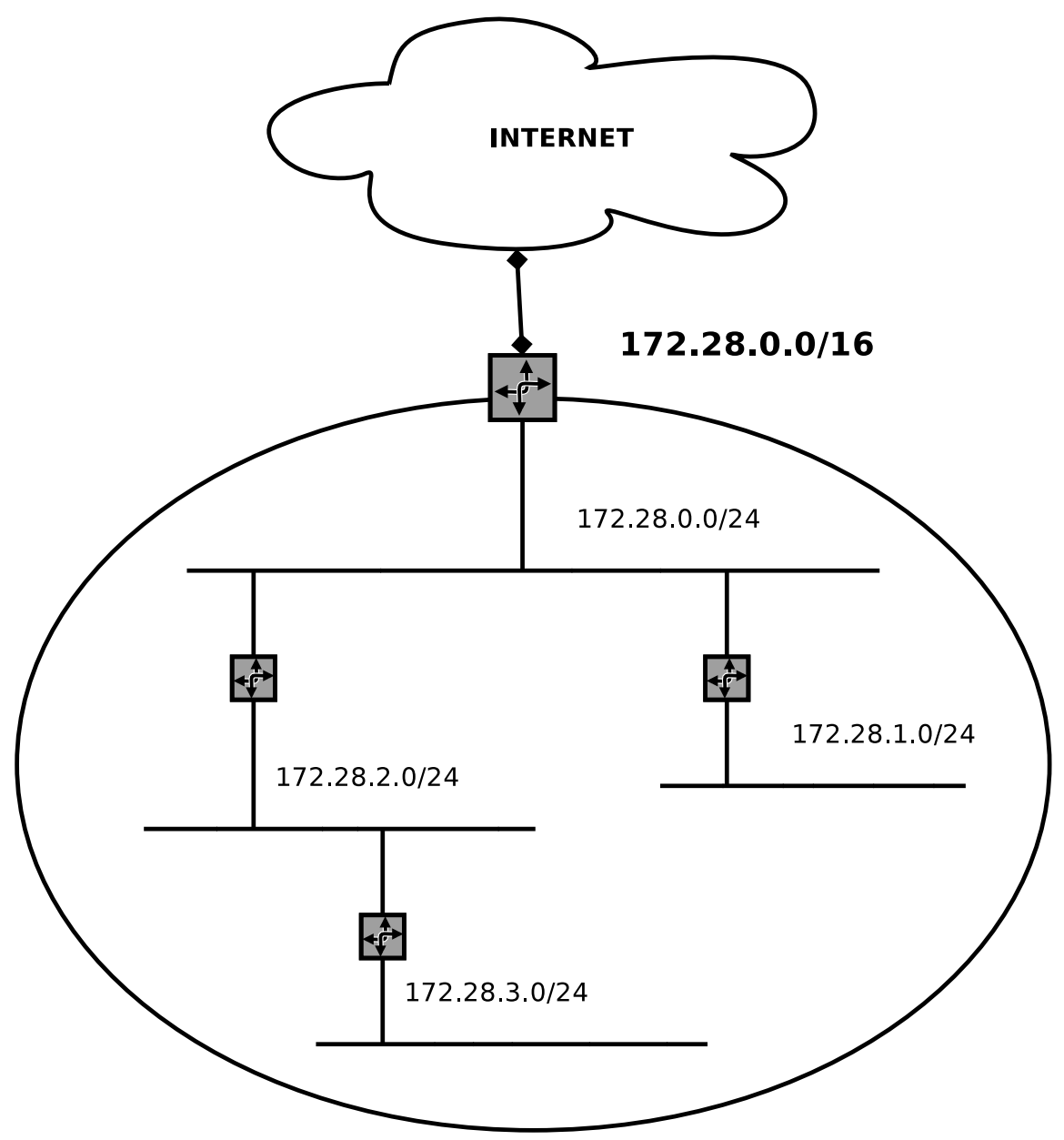

Figura 2.4: Divisão em sub-redes

fazendo com que ela seja composta por duas redes classe C. Isso traz duas grandes vantagens: primeiro, instituições cujo porte não necessite de uma rede classe B e precisem de mais de 254 endereços IP, podem utilizar duas redes classe C; e segundo, as tabelas de roteamento tornam-se bem menores com esse tipo de representação, pois várias redes contínuas que possuam o mesmo nexthop podem ser representadas em um único registro.

O mecanismo de redes com endereços IP privados permite a criação de redes internas isoladas da Internet. Algumas faixas de endereços foram destinadas à criação desse tipo rede [10]:

- Classe A: 10.0.0.0/8

- Classe B: 172.16.0.0/12 
- Classe C: 192.168.0.0/16

Essas faixas são de uso exclusivo para redes IP internas, ou seja, não devem trafegar na Internet. Os roteadores conectados à Internet devem rejeitar datagramas com endereço de origem ou destino pertencentes a essas faixas. A comunicação externa é feita através de servidores proxy ou roteadores capazes de realizar conversões de endereços. Essas conversões são conhecidas como Network Address Translation (NAT) [11].

A utilização de redes IP privadas teve um efeito colateral positivo: segurança. A criação dessas redes ofereceu naturalmente um ambiente mais isolado e seguro, pois roteadores intermediários na Internet devem descartar todo datagrama que tenha IP privado como origem e/ou destino.

\subsection{Cabeçalho}

O datagrama IP é dividido em duas partes: cabeçalho e dados.

O cabeçalho contém as informações administrativas do datagrama, como origem, destino, tamanho, entre outras [12]. O campo de dados contém as informações das aplicações (figura 2.5).

O cabeçalho IP consiste em uma estrutura de, no mínimo, 20 octetos, organizados da seguinte maneira:

VERS, 4 bits: versão do protocolo IP (atualmente, pode ser 4 ou 6). Este campo é utilizado para definir o formato do cabeçalho.

HLEN, 4 bits: tamanho do cabeçalho, em unidades de 32 bits (word). Os cabeçalhos mais comuns (sem opções e sem preenchimento) possuem 20 octetos, ou seja, HLEN igual a 5.

Service Type, 8 bits: este campo é utilizado pelos roteadores para determinar como o datagrama deve ser tratado (figura 2.6). Na primeira definição deste 


\begin{tabular}{|c|c|c|c|c|}
\hline 0 & 4 & 8 & 16 & 19 \\
\hline VERSION & HLEI & TYPE & TOTAL & ENGTH \\
\hline \multicolumn{3}{|c|}{ IDENTIFICATION } & FLAGS & FRAGMENT OFFSET \\
\hline$\underline{\pi L}$ & & PROTOCOL & \multicolumn{2}{|c|}{ HEADER CHECKSUM } \\
\hline \multicolumn{5}{|c|}{ SOURCE IP ADDRESS } \\
\hline \multicolumn{5}{|c|}{ DESTINATION IP ADDRESS } \\
\hline \multicolumn{3}{|c|}{ IP OPTIONS } & & PADDING \\
\hline \multicolumn{5}{|l|}{$\underline{\text { DATA }}$} \\
\hline \multicolumn{5}{|l|}{ DATA } \\
\hline \multicolumn{5}{|c|}{. I I } \\
\hline DATA & & & & \\
\hline
\end{tabular}

Figura 2.5: Datagrama IP

campo [12], os três primeiros bits (Precedence) representam a prioridade do datagrama, que varia entre zero (normal) e 7 (controle de rede). Este mecanismo é importante para o gerenciamento da rede, pois mesmo que ela esteja congestionada por dados de usuário, os roteadores dão prioridade para datagramas com o PRECEDENCE maiores. Os três bits seguintes representam o tipo de transporte: $\mathrm{D}=$ baixo delay, $\mathrm{T}=$ alto throughput $\mathrm{e} \mathrm{R}=$ alta confiabilidade. $\mathrm{O}$ protocolo $\mathrm{IP}$ não garante o tipo de conexão desejada, portanto, os bits $\mathrm{D}, \mathrm{T}$ e R funcionam como uma informação adicional: se um roteador tem duas rotas para o destino do datagrama, ele pode utilizar os valores desses bits para decidir por qual rota encaminhá-lo. Os últimos 2 bits não são utilizados.

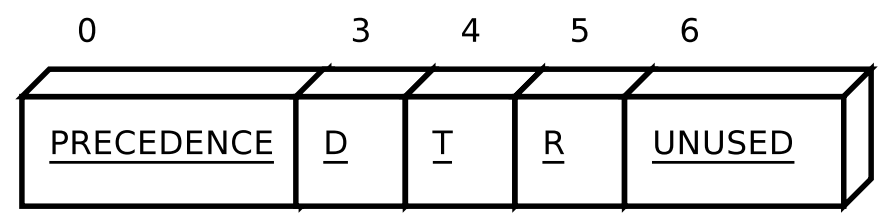

Figura 2.6: TOS com os bits D, T e R 
Em [13], os bits D, T, R e o primeiro do campo não utilizado passaram a fazer parte do subcampo Type of Service (TOS) (figura 2.7). Dessa maneira, apenas o oitavo bit não é utilizado. Esses 4 bits passam a ser tratados como um único campo, e seus valores e significados são os seguintes:

- 1000 - baixo atraso;

- 0100 - largura de banda alta;

- 0010 - alta confiabilidade;

- 0001 - mínimo custo financeiro;

- 0000 - serviço normal.

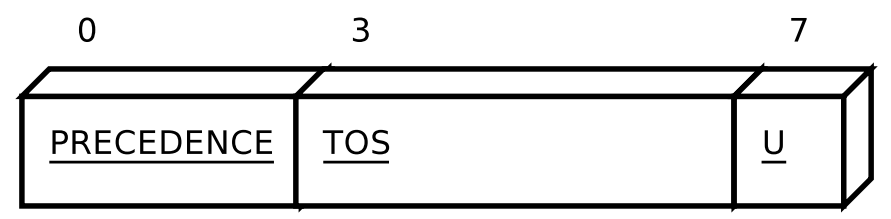

Figura 2.7: TOS unificado

Na década de 90, o Internet Engineering Task Force (IETF) redefiniu o significado desse campo: os 6 primeiros bits passaram a representar o tipo de serviço e, novamente, os 2 últimos bits foram definidos como não utilizados. Com essa nova interpretação, esse campo passou a ser conhecido como differentiated services [14] ou serviços diferenciados (figura 2.8).

Na definição do campo TOS como serviços diferenciados, os 6 bits mais significativos devem ser interpretados como um campo único, não-estruturado, e os 2 bits menos significativos devem ser ignorados.

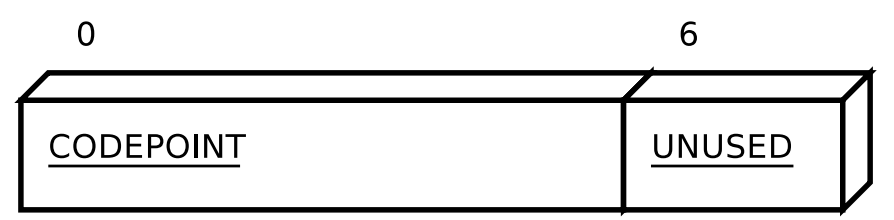

Figura 2.8: DSCP 
Atualmente, os bits 6 e 7 estão definidos para suporte à Explicit Congestion Notification (ECN) [15].

Para oferecer um certo nível de compatibilidade entre o sistema de serviços diferenciados e a definição do campo TOS, existem alguns valores do campo Differentiated Services Code Point (DSCP) que são mapeados para comportamentos (conhecidos como Per Hop Behavior (PHB)) padronizados [16].

Valores de DSCP no formato 11X000 devem ter prioridade maior que 000000, respeitando assim, a semântica do campo PRECEDENCE: valores altos (6 e 7) têm prioridade sobre os valores menores. Valores no formato XXXXX0 são reservados ao IETF, enquanto que valores no formato XXXX11 e XXXX01 estão disponíveis para uso local ou experimental.

Total Length, 16 bits: tamanho total do datagrama IP, em octetos. Com os valores deste campo e do campo HLEN, é possível obter o tamanho do campo de dados (basta subtrair HLEN de Total Length).

Identification, 16 bits: identificação do datagrama, utilizado na remontagem de fragmentos. Quando um datagrama é maior do que o Maximum Transfer Unit (MTU) de uma determinada rede local, ele precisa ser dividido em fragmentos para que possa ser transmitido nessa rede. Nessa fragmentação, cada parte fragmentada torna-se um novo datagrama IP, e a maior parte dos dados do cabeçalho original é replicada nos cabeçalhos dos datagramas, inclusive o campo Identification. Dessa maneira, pode-se saber a qual datagrama cada fragmento pertence.

Flags, 3 bits: o primeiro bit do campo FLAGS é reservado e o seu valor deve ser 0. O segundo é conhecido como bit Do not fragment (DF), e determina se o datagrama pode ou não ser fragmentado. Se o seu valor for 1 (ativado) e o datagrama for maior que o MTU da rede local, o roteador deve descartá-lo e enviar uma mensagem de erro informando que é necessário fragmentar o datagrama, mas 
a fragmentação não foi possível porque o bit DF está ativado. O terceiro bit é conhecido como bit More Fragments (MF), e indica se um datagrama contém o último fragmento ou não do datagrama original. Esse bit é necessário para se saber o tamanho do datagrama original, já que cada novo datagrama carrega apenas o tamanho de cada fragmento. Com os valores dos campos Fragment Offset e Total Length do datagrama que tem o bit MF em zero é possível calcular o tamanho do datagrama original.

Offset, 13 bits: o tamanho de cada fragmento é definido de acordo com o MTU da rede onde irá trafegar. Todos os fragmentos, com exceção do último, recebem uma porção dos dados de mesmo tamanho, MTU - HLEN, e conseqüentemente, valores de OFFSET múltiplos de MTU - HLEN. O primeiro fragmento possui Offset $=0 *(\mathrm{MTU}-\mathrm{HLEN})$, o segundo, Offset $=1 *(\mathrm{MTU}-\mathrm{HLEN})$ e assim por diante. A maioria dos datagramas não tem o tamanho do campo de dados como múltiplo de (MTU - HLEN). Como conseqüência, o último fragmento geralmente é menor que os demais. Cada unidade desse campo representa 16 octetos.

TTL, 8 bits: este campo funciona como um contador, que limita o tempo de vida de um datagrama na Internet. Ao receber um datagrama, todo roteador deve analisar o campo Time to Live (TTL). Se o seu valor for maior que zero, ele deverá diminuí-lo em uma unidade, e se for igual a zero, esse datagrama deve ser descartado. Com isso, evita-se que datagramas trafeguem indefinidamente pela Internet.

Protocol, 8 bits: especifica a qual protocolo pertence os dados carregados no campo Data. Como exemplo podemos citar TCP (6), UDP (17) e ICMP (1).

Header Checksum, 16 bits: é utilizado para garantir a integridade do cabeçalho IP. O campo de dados não é abrangido por essa checagem. Isso alivia a carga de processamento em roteadores, que precisam verificar apenas a integri- 
dade do cabeçalho IP. Para calcular o valor desse campo, é necessária a utilização de todos os campos do cabeçalho, inclusive o próprio Header Checksum, por isso assume-se que ele tem como valor inicial zero.

SRC IPADDR, 32 btis: endereço de origem do datagrama.

DST IPADDR, 32 bits: endereço de destino do datagrama.

Options, 0 a 320 bits: O tamanho de 4 bits do campo HLEN pode representar, no máximo, 15 unidades de 32 bits, que equivale a 480 bits. O menor tamanho de cabeçalho possível (sem opções) ocupa 160 bits. Conseqüentemente, restam apenas 40 octetos (320 bits) para esse campo, que é uma quantidade pequena para alguns de seus propósitos (como exemplo, podemos citar a definição da rota a ser seguida, também conhecida como source routing).

\subsection{Roteamento}

Todo datagrama de rede que sai do equipamento origem em direção ao seu destino tem um caminho a seguir. O processo de definição desse caminho é conhecido como roteamento.

A figura 2.9 ilustra um cenário de rede que será utilizada como exemplo para as discussões a seguir.

Os equipamentos conectados à Internet (estações de trabalho e roteadores) possuem uma tabela de roteamento que contém informações necessárias para alcançar o endereço destino ou próximo nó intermediário.

A primeira solução "intuitiva" seria fazer com que cada equipamento na Internet tivesse uma tabela completa, com todas as rotas para todos os destinos possíveis. A tabela 2.1 ilustra um exemplo configurado em R1. Essa solução é inviável por dois motivos: primeiro, seria necessária uma capacidade de armazenamento muito grande para se ter todos os registros localmente; e, segundo, 


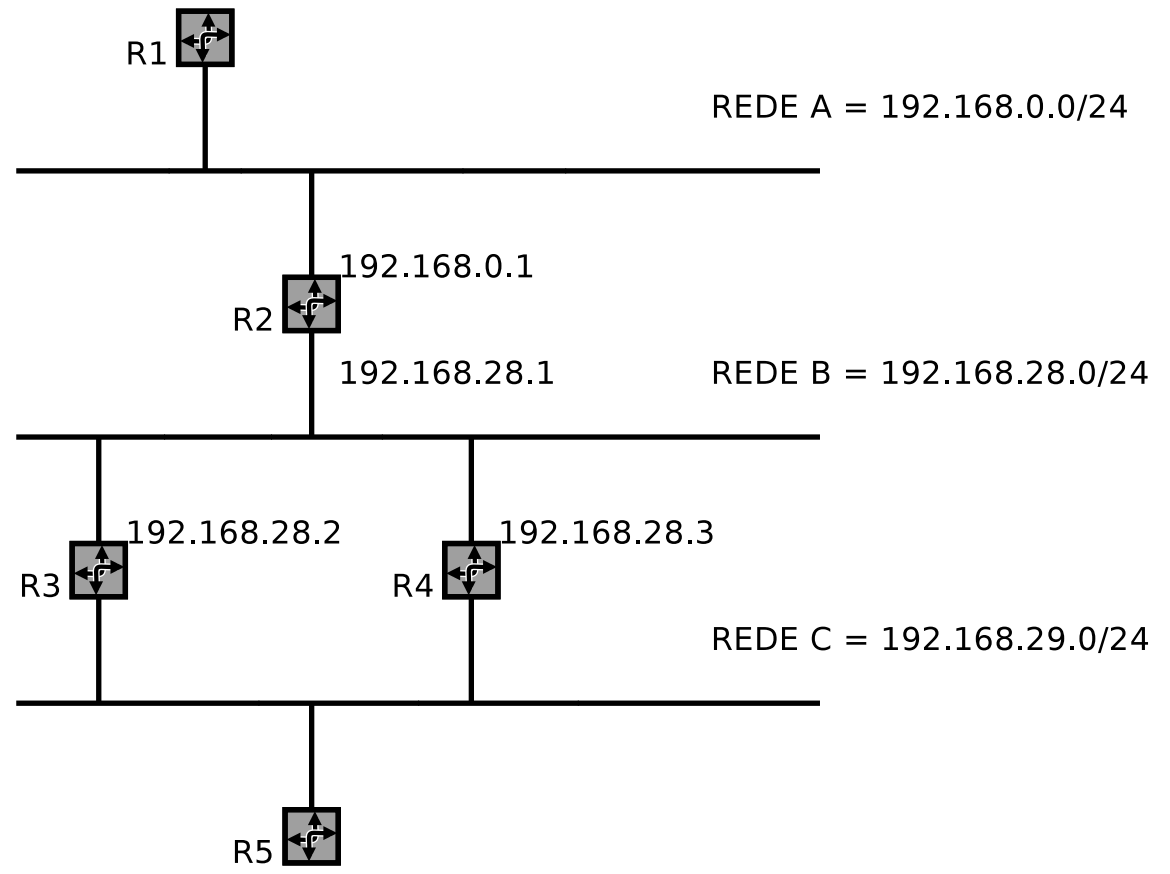

Figura 2.9: Duas opções de ligação entre as redes B e C

seria necessário um alto poder de processamento para consultar e atualizar os registros constantemente, mantendo a consistência das rotas entre os roteadores e estações.

Para diminuir a necessidade de processamento e armazenamento, as tabelas de rotas são criadas com base nas informações de rede, e não de nó. Dessa maneira, a quantidade de registros na tabela de roteamento diminui drasticamente (tabela 2.2), diminuindo também a demanda por processamento e armazenamento.

Mesmo com a utilização de endereços de rede, a tabela teria um tamanho considerável se tivesse as rotas para todas as redes existentes. As estações e roteadores (com exceção dos roteadores centrais) passaram, então, a ter uma rota especial, conhecida como rota padrão, que corresponde a todos os casos que não têm uma rota explícita definida na tabela de roteamento (tabela 2.3).

É importante notar que, em todos os casos, R1 tem na tabela de rotas R2 como roteador tanto para a rede $\mathrm{B}$ como para a rede C. R1 não utiliza R3 (nem R4, se for o caso) para a rede C. Este procedimento tem como objetivo facilitar a administração da Internet através da descentralização do gerenciamento da rede. 
Tabela 2.1: Tabela de rotas de R1 utilizando endereços individuais

\begin{tabular}{|l|l|}
\hline Destino & Roteador de saída \\
\hline 192.168 .0 .0 & Interface local \\
192.168 .28 .5 & 192.168 .0 .1 \\
192.168 .28 .35 & 192.168 .0 .1 \\
192.168 .28 .44 & 192.168 .0 .1 \\
192.168 .28 .51 & 192.168 .0 .1 \\
192.168 .28 .98 & 192.168 .0 .1 \\
192.168 .28 .200 & 192.168 .0 .1 \\
192.168 .28 .253 & 192.168 .0 .1 \\
192.168 .29 .7 & 192.168 .0 .1 \\
192.168 .29 .9 & 192.168 .0 .1 \\
192.168 .29 .27 & 192.168 .0 .1 \\
192.168 .29 .39 & 192.168 .0 .1 \\
192.168 .29 .40 & 192.168 .0 .1 \\
192.168 .29 .199 & 192.168 .0 .1 \\
192.168 .29 .210 & 192.168 .0 .1 \\
\hline
\end{tabular}

Tabela 2.2: Tabela de rotas de $\mathrm{R} 1$ utilizando endereços de rede

\begin{tabular}{|l|l|}
\hline Destino & Roteador de saída \\
\hline $192.168 .0 .0 / 24$ & Interface local \\
$192.168 .28 .0 / 24$ & 192.168 .0 .1 \\
$192.168 .29 .0 / 24$ & 192.168 .0 .1 \\
\hline
\end{tabular}

A seguinte situação ilustra a vantagem de se utilizar sempre roteadores da mesma rede. Inicialmente, as redes B e C são interligadas pelo roteador R3, e a tabela incorreta de roteamento de R1 está ilustrada na tabela 2.4.

Uma falha em R3 envolve diretamente as redes B e C, e as medidas locais a serem tomadas seriam:

- Configurar R4 como roteador de ligação das redes B e C;

- Reconfigurar R2 para utilizar $\mathrm{R} 4$ como roteador para a rede $\mathrm{C}$;

- Reconfigurar R5 para utilizar R4 como roteador para a rede B.

Após as manutenções anteriores, a rede $\mathrm{C}$ tornar-se-ia inacessível para $\mathrm{R} 1$, já que este tem R3 (que está inativo) como roteador para a rede C. Se R1 utilizasse $\mathrm{R} 2$ como roteador para a rede $\mathrm{C}$, a manutenção em $\mathrm{R} 2$ já restauraria a comunicação entre $\mathrm{R} 1$ e a rede $\mathrm{C}$. 
Tabela 2.3: Tabela de rotas de R1 utilizando uma rota padrão

\begin{tabular}{|l|l|}
\hline Destino & Roteador de saída \\
\hline $192.168 .0 .0 / 24$ & Interface local \\
$0.0 .0 .0 / 0$ & 192.168 .0 .1 \\
\hline
\end{tabular}

Tabela 2.4: Tabela incorreta de rotas em R1

\begin{tabular}{|l|l|}
\hline Destino & Roteador de saída \\
\hline REDE A & Interface local \\
REDE B & 192.168 .0 .1 \\
REDE C & 192.168 .28 .2 \\
\hline
\end{tabular}

Esta é uma característica importante do roteamento IP: cada registro da tabela de roteamento tem como próximo nó um roteador que está na mesma rede, proporcionando uma maior consistência nas rotas. É importante notar que nenhum dos equipamentos que participam da transmissão do datagrama tem conhecimento de toda a rota utilizada: cada um deles confia a entrega do datagrama ao nó roteador seguinte.

Na tabela de roteamento, a decisão da rota é feita com base na coincidência mais longa: a prioridade é para rotas de host, seguidas por rotas em ordem decrescente de máscaras (quanto mais bits o netid de uma rede ocupa, maior é a sua prioridade na escolha como rota a ser seguida), e por último, a rota default.

De forma simplificada, o algoritmo de roteamento IP [17] pode ser definido da seguinte maneira:

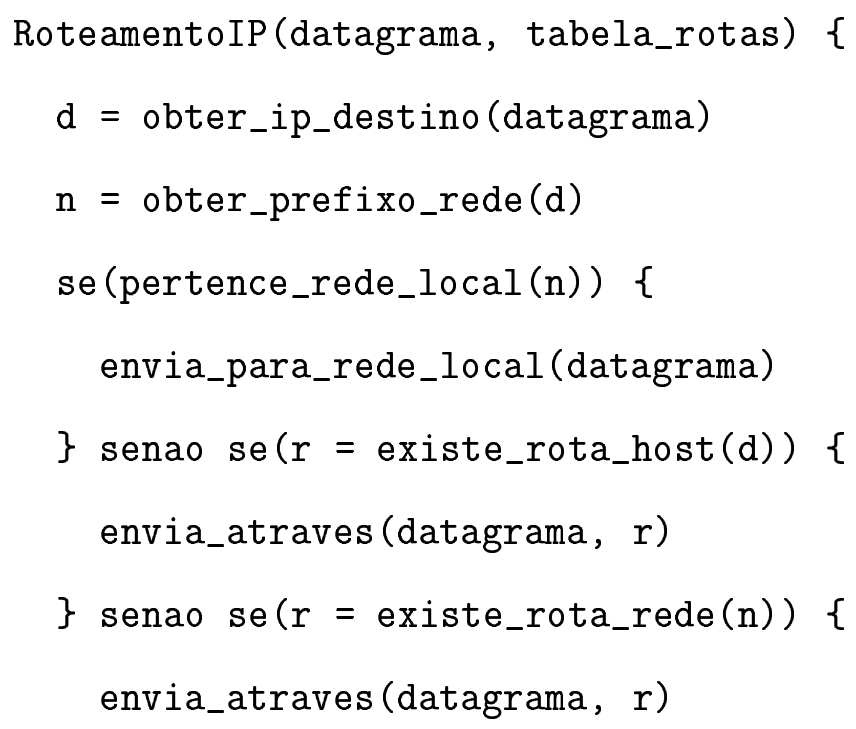




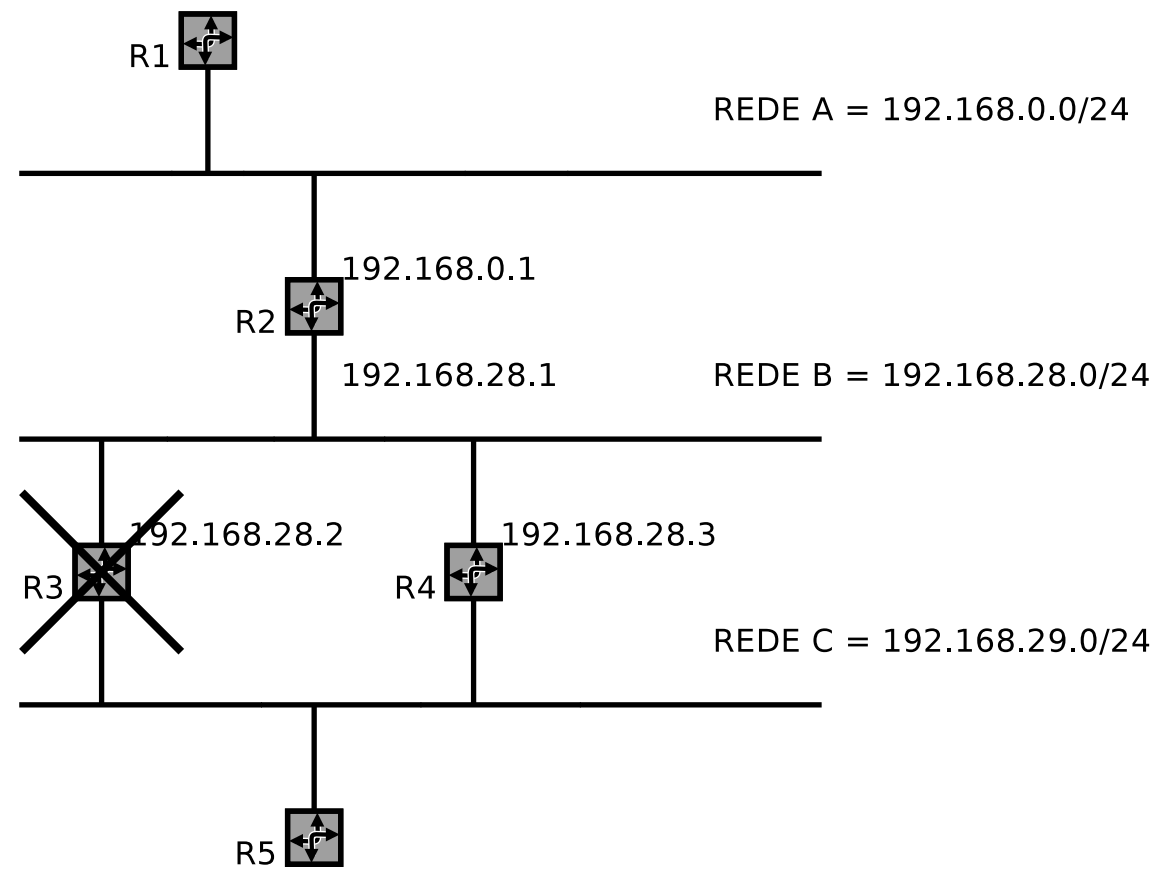

Figura 2.10: Falha no roteador R3: R4 passa a ligar as redes B e C

\} senao se(r = existe_rota_padrao()) \{

envia_atraves (datagrama, $r$ )

\} senao \{

retorna(erro)

\}

\}

Além dos campos de endereço origem e destino, é importante lembrar que outros campos são analisados no processo de roteamento IP, e determinam se o datagrama é ou não encaminhado, assim como a sua prioridade de reencaminhamento:

- O campo VERSION é o primeiro que deve ser analisado. Ele determina o formato do cabeçalho IP e, conseqüentemente, como extrair as informações de todos os outros campos;

- O campo TTL determina a continuidade do datagrama, prevenindo a formação de loops; 
- O campo TOS possibilita uma diferenciação no tratamento dos datagramas, oferecendo uma espécie de qualidade de serviço;

- O campo FLAGS possui o bit DF, que determina se é proibida (valor 1) ou não (valor 0) a fragmentação do datagrama IP.

Roteamento Dinâmico: Em redes de grande porte, com um número grande de roteadores, a operação manual de cada um deles torna-se inviável, devido ao tempo de resposta às mudanças e à grande possibilidade de erro por parte dos operadores. Esse fato levou à criação de protocolos para a automatização das manutenções dos roteadores.

Os dois principais algoritmos de propagação de rotas são Distance Vector Routing e Link State Routing [18].

No primeiro tipo (Distance Vector Routing), os roteadores trocam entre si mensagens contendo endereços de rede e as respectivas distâncias. Quando um roteador recebe uma mensagem, ele compara a sua tabela de roteamento com a tabela recebida nessa mensagem. Se na tabela recebida houver algum caminho mais curto para uma determinada rede, ou se a distância para uma rede conhecida foi alterada, o roteador atualiza a sua tabela de roteamento com os dados recebidos. Nesse tipo de algoritmo foram detectados três grandes problemas. O primeiro é a fraca escalabilidade, pois o tamanho e quantidade de mensagens são proporcionais à quantidade de redes existentes. O segundo é a demora na convergência, pois cada roteador depende dos outros para calcular a distância a uma determinada rede. E por último, o valor das distâncias não considera a capacidade dos links. Como exemplo deste tipo de algoritmo, podemos citar o Border Gateway Protocol (BGP) [19].

O segundo tipo funciona de uma maneira diferente. Cada roteador possui uma tabela de rotas da rede. Para a construção desse mapeamento, as mensagens trocadas não possuem destinos e suas respectivas distâncias, e sim, o status das conexões locais de cada roteador. Essa diferença cobre duas deficiências do 
algoritmo anterior: melhora a escalabilidade, pois o tamanho das mensagens independe da quantidade de redes, e diferenciação de links, pois o status de uma conexão pode conter informações sobre a sua capacidade, além de outras características. Como exemplo deste tipo de algoritmo, podemos citar o Open Shortest Path First (OSPF) [20].

\subsection{Qualidade de Serviço}

Para ter um desempenho satisfatório, as aplicações exigem uma capacidade mínima de recursos, sejam em processamento, armazenamento ou rede. Além disso, cada tipo de aplicação tem as suas particularidades. Por exemplo, o sistema de correspondências eletrônicas (email) exige confiabilidade, isto é, integridade dos dados trafegados, mas não exige baixo atraso (por não ser uma aplicação online e interativa) nem largura de banda (as mensagens são pequenas). Já uma vídeoconferência exige atrasos pequenos (interatividade) e largura de banda grande (devido à quantidade de dados trafegados por unidade de tempo), mas pouca confiabilidade (alguns bits corrompidos geram pequenos erros nas imagens, o que é aceitável até certo ponto) [21].

Oferecer qualidade de serviço - QoS - significa garantir um desempenho mínimo de uma rede, nos quesitos largura de banda, atraso, variação de atraso (jitter) e perda. A seguir serão analisadas algumas técnicas que contribuem para se obter uma boa qualidade de serviço.

Superdimensionamento: O método mais simples para se oferecer uma boa qualidade de serviço é implantar uma infra-estrutura capaz de atender a todas as demandas. Dessa maneira, não é necessário se preocupar com racionalização de recursos: simplesmente há mais do que o necessário (exceto nas situações de rajadas, nas quais a rede pode tornar-se saturada por alguns momentos). A desvantagem dessa solução é o seu alto custo. 
Armazenamento em memória: O armazenamento em memória consiste em fazer com o que o receptor (cliente) armazene os dados antes de processá-los. Essa técnica não ataca os problemas de confiabilidade nem de largura de banda, além de aumentar o atraso. O benefício trazido pela buferização é a eliminação (ou pelo menos a diminuição) da variação do atraso (jitter), melhorando o desempenho de aplicações de exibição de vídeo, por exemplo.

Controle de tráfego: A buferização é um processo que ocorre no receptor dos dados (cliente). Já o controle de tráfego é feito no servidor, e controla a quantidade de dados que é enviada aos clientes. A quantidade de recursos disponíveis é combinada anteriormente entre os usuários e os provedores de serviço. Essa prática reduz o congestionamento, possibilitando que seja oferecida a quantidade de recursos acordada previamente.

Engenharia de Tráfego: Uma prática interessante que favorece a obtenção de qualidade de serviço é a utilização mais racional dos recursos da rede. Como uma rede é uma malha, com várias rotas possíveis para a comunicação entre dois pontos, pode-se elaborar desvios de tráfego dos dados para caminhos alternativos. Isso pode ser feito para evitar pontos de congestionamento e até mesmo direcionar determinados tipos de dados para rotas mais adequadas. Para garantir um limite mínimo, é possível solicitar aos roteadores a reserva de recursos necessários para uma determinada aplicação, criando uma espécie de circuito virtual. Para que esse circuito seja criado, todos os roteadores participantes devem concordar em reservar todos os recursos necessários.

A rota que deve ser seguida para garantir essa qualidade de serviço é definida através da comunicação entre os roteadores por meio de protocolos de reserva de recursos. Um dos protocolos de reserva mais utilizados é o Resource Reservation Protocol (RSVP) [22]. No protocolo RSVP, uma das extremidades da conexão envia uma mensagem para determinar a rota na qual todos os roteadores ofere- 
çam os recursos necessários. Se algum roteador negar o pedido por recursos, o nó de origem da mensagem recebe uma resposta negativa, e se todos os roteadores aceitarem esse pedido, a rota pode ser utilizada. A criação desses caminhos deve ser a mais racional possível, evitando que alguns roteadores fiquem sobrecarregados e outros, ociosos. Essa prática é conhecida como Engenharia de Tráfego [23].

A criação de circuitos oferece uma boa qualidade de serviço, mas tem seus pontos negativos. A criação inicial do circuito é um processo pesado e relativamente demorado, e pode se tornar um ponto crítico em um cenário com muitos fluxos. Outro problema com circuitos é a vulnerabilidade à falha de um ou mais roteadores que fazem parte desse circuito. Como há uma reserva inicial de recursos, qualquer alteração na rota do circuito sem uma nova verificação dos recursos disponíveis pode comprometer a qualidade de serviço oferecida.

Como alternativa à obtenção de qualidade de serviço baseada em fluxo, tem-se a qualidade de serviço baseada em classes de tráfego. Nesse sistema, não há uma reserva de recursos para cada conexão, mas sim para grupos de conexões, os quais podem ser formados considerando-se as necessidades por recursos das aplicações.

Atualmente, o protocolo IP oferece um certo nível de qualidade de serviço através do campo TOS do seu cabeçalho. Os roteadores utilizam o valor desse campo para dar diferentes tipos de tratamentos aos datagramas IP. Esse sistema de tratamento diferenciado é conhecido como DiffServ [24], e foi criado como uma alternativa ao Integrated Services [25].

Roteadores que suportam o sistema de serviços diferenciados são conhecidos como $D S$-compliant nodes. Esses roteadores são capazes de interpretar o campo TOS e com base no seu valor, oferecer tratamentos diferenciados (PHB) para os datagramas IP.

A atribuição de valores a esse campo pelos roteadores é livre para a administração de cada domínio de rede.

Existem datagramas que passam por vários domínios antes de alcançar o seu 
destino. Para possibilitar um certo grau de interação entre esses domínios e criar um ambiente de rede relativamente homogêneo, o IETF criou alguns PHBs padrão, independentes do domínio de rede. Dois exemplos de PHBs padronizados são Expedited Forwarding (EF) [26] e Assured Forwarding ( $A F$ ) [27].

O PHB EF oferece duas classes de serviços: normal e prioritário. Datagramas marcados como prioritários devem trafegar pelos roteadores como se a rede fosse dedicada a esse tipo de datagrama, mesmo havendo congestionamento de datagramas marcados como normais. Uma possível implementação desse sistema é a criação de duas filas nos roteadores, uma para datagramas normais e outra para datagramas prioritários.

No PHB AF existem quatro diferentes classes de serviço. Em cada classe, os datagramas podem ser diferenciados quanto à preferência de descarte: baixa, média e alta. Combinando-se as quatro classes com as três políticas de descarte, é possível a criação de doze classes de serviço distintas (tabela 2.5).

Tabela 2.5: AF: 12 possíveis classes de serviço

\begin{tabular}{|c|c|c|c|c|}
\hline Possibilidade de Descarte & Classe 1 & Classe 2 & Classe 3 & Classe 4 \\
\hline Baixa & 001010 & 010010 & 011010 & 100010 \\
Média & 001100 & 010100 & 011100 & 100100 \\
Alta & 001110 & 010110 & 011110 & 100110 \\
\hline
\end{tabular}

Um roteador pode atribuir qualquer valor ao campo TOS. Portanto, para que uma rede utilize adequadamente os serviços diferenciados, é necessário que os seus roteadores de borda isolem essa rede, policiando e atribuindo valores adequados a esse campo. Se esse cuidado não for tomado, os roteadores internos podem interpretar o campo DSCP com um valor incorreto e a rede pode apresentar comportamentos inesperados.

\section{$2.5 \quad \mathrm{ICMP}$}

Existem protocolos que são amplamente utilizados como ferramentas de auxílio no gerenciamento de uma rede TCP/IP. 
O protocolo ICMP [28] tem como principal tarefa padronizar a troca de informações de controle entre os equipamentos, já que o IP não oferece mecanismos que auxiliem em testes de conexão ou detecção de falhas.

As mensagens ICMP trafegam dentro do campo de dados de um datagrama IP, da mesma maneira que dados de aplicações de usuário, e não recebem nenhum tratamento ou prioridade especial por parte do protocolo IP, mesmo sendo um protocolo de controle. Como conseqüência, mensagens ICMP podem ser perdidas ou descartadas, e se uma rede já está congestionada, as mensagens ICMP podem agravar ainda mais o cenário.

Uma das funções mais utilizadas do protocolo ICMP é a verificação de conectividade entre roteadores e estações de uma rede IP. As mensagens do tipo echo request e echo reply [28] são utilizadas para verificação de conectividade. Caso haja problemas na comunicação entre os dois nós, o protocolo ICMP possibilita o envio de datagramas com mensagens que auxiliam na detecção e até mesmo no tratamento do erro. Como exemplo podemos citar a mensagem do tipo Destination Unreachable: além de informar à origem do datagrama que determinado destino IP não pode ser alcançado, esse tipo de mensagem informa o motivo (rede fora de alcance, fragmentação necessária mas o bit DF está ativado, entre outros) [17].

O protocolo ICMP possui mensagens que permitem o redirecionamento de estações da mesma rede IP para rotas mais adequadas. Se um roteador RA recebe um datagrama de $\mathrm{H}$, cuja saída é um segundo roteador RB que está na mesma rede IP, RA encaminha o datagrama para o seu destino e envia uma mensagem ICMP do tipo redirect para $\mathrm{H}$, contendo o endereço do roteador adequado (RB), possibilitando que $\mathrm{H}$ corrija sua tabela de roteamento. 


\section{Capítulo 3}

\section{MPLS: Conceitos fundamentais}

$M P L S$ é uma tecnologia aberta que combina roteamento de nível 3 com chaveamento de nível 2 para realizar o encaminhamento de datagramas, através de pequenos "rótulos" de tamanho fixo. Esses rótulos são números utilizados no protocolo MPLS para decidir através de qual interface encaminhar o datagrama [29].

Os principais motivos que levaram ao desenvolvimento de uma tecnologia baseada em rótulos foram o menor processamento para o encaminhamento e a maior possibilidade de gerenciamento do tráfego de dados. A redução no processamento ocorre porque o rótulo é uma informação simples, pequena e de tamanho fixo, ao contrário de um cabeçalho de rede. O cabeçalho MPLS tem 32 bits, enquanto um cabeçalho IP tem, no mínimo, 20 octetos. Além de ter tamanho maior, a utilização do cabeçalho IP é mais complexa, pois ele é dividido em vários campos que precisam ser analisados individualmente no processo de roteamento. Outro processo significativo em relação à carga de processamento é a checagem de integridade (checksum) realizada pelo IP em todos os nós da rota seguida, devido ao decremento do valor de TTL em cada roteador. No MPLS, essa checagem não é executada. A melhora no gerenciamento de tráfego é obtida graças à um mecanismo eficiente que possibilita a determinação da rota seguida pelos dados mesmo em uma rede sem conexão (que é o caso do IP). 
Algumas empresas começaram a desenvolver tecnologias baseadas na utilização de rótulos. Como exemplos, podemos citar IP Switching (Ipsilon) [30], Cell Switching Router (Toshiba), Tag Switching Architecture (CISCO) [31], ARIS (IBM) e IP Navigator (Ascend) [32]. Como a utilização dessa tecnologia começou a crescer, foi notada a necessidade de uma padronização para a indústria.

O primeiro encontro do MPLS Working Group aconteceu em Abril de 1997, durante o $38^{\circ}$ Encontro IETF em Memphis, Tenessee [33]. A partir de então, o MPLS WG passou a trabalhar na definição do protocolo MPLS, além dos procedimentos e protocolos para a distribuição dos rótulos entre os roteadores.

O MPLS é considerado uma ligação entre as camadas 2 e 3 , pois ele utiliza o sistema de endereçamento dos protocolos de nível 3 e o sistema de transmissão em redes locais já existentes. Por estar nessa posição intermediária (entre as camadas 2 e 3), o protocolo MPLS pode ser considerado um protocolo de camada 2.5 [33].

Por ser uma camada de integração, é fundamental que ela seja compatível com o maior número possível de protocolos da camada 3 e tecnologias da camada 2. Essa característica justifica o nome "MultiProtocol" do MPLS.

Outra característica importante do protocolo MPLS é o suporte a várias granularidades de encaminhamento de datagramas. Em um extremo, temos a caracterização dos datagramas IP considerando-se apenas a informação da rede à qual pertence o endereço de destino. No outro, a caracterização pode ser feita considerando-se informações da camada de transporte (portas TCP, por exemplo). O primeiro tipo de granularidade (grosseira) é importante para a escalabilidade de uma rede MPLS: uma única caracterização pode tratar vários tipos de datagramas, diminuindo a carga de processamento no momento da consulta à tabela de encaminhamento. O segundo tipo de granularidade (fina) é importante no oferecimento de qualidade de serviço: é possível desviar dados interativos (voz, vídeo, terminais) para caminhos com menor atraso, e dados não interativos (emails, transferência de arquivos) para caminhos com maior largura de banda, por exemplo. 
A arquitetura IP sobre MPLS também pode ser comparada ao sistema de trilhos e vagões motorizados, utilizado para ilustrar o funcionamento do protocolo IP. Cada vagão representa o datagrama IP rotulado, ou seja, o datagrama IP encapsulado pelo cabeçalho MPLS. Os trilhos continuam representando os meios físicos de comunicação, e as encruzilhadas com os operadores representam os roteadores MPLS. Os vagões passam a exibir uma placa mais simples, que contém o valor do rótulo. O papel dos operadores é analisar o valor na placa, decidir para onde desviar o vagão, inserir um novo valor na placa e, por último, enviar o vagão para o trilho correto. Uma diferença importante é que nesta situação, o operador da encruzilhada não conhece o endereço destino do vagão. A única informação que ele tem é para qual trilho ele deve encaminhar o vagão. Dessa maneira, o operador não precisa ter um mapa da malha de trilhos para efetuar os desvios corretamente.

\subsection{Elementos de uma rede MPLS}

A arquitetura IP sobre MPLS traz novos conceitos em relação ao roteamento IP convencional e, conseqüentemente, novas figuras e funcionalidades surgem no cenário de rede. Seguem abaixo algumas nomenclaturas essenciais para uma discussão sobre o protocolo MPLS, que podem ser encontradas em [29]:

Label Switched Path (LSP): consiste no caminho entre o nó de entrada (ingress node), possíveis nós intermediários (transit nodes) e nó de saída (egress node) de uma rede MPLS em um mesmo nível hierárquico. Um conjunto de nós MPLS contínuos é chamado de domínio MPLS, geralmente controlado por uma única administração.

Forwarding Equivalence Classes (FEC): é um conjunto de datagramas de rede que são encaminhados da mesma maneira, isto é, pelo mesmo LSP. Cada roteador MPLS deve ter uma tabela contendo uma FEC para cada tipo de da- 
tagrama. Várias características podem ser utilizadas na definição das FECs: endereços de rede, portas TCP ou UDP, DSCP.

Label Switching Router (LSR): é um equipamento (também chamado de nó ou roteador) capaz de realizar encaminhamentos de datagramas de rede através de rótulos MPLS. Existem três tipos de LSR: Ingress LSR, Transit LSR e Egress LSR.

- Ingress LSR: é o LSR de entrada de uma rede MPLS. Nele é feita a atribuição inicial dos rótulos para cada datagrama que vai entrar no domínio MPLS. Os ingress Label Edge Router (LER) analisam as informações do cabeçalho de rede e associam cada datagrama a uma FEC, e toda FEC tem um rótulo associado que será utilizado no encaminhamento para o próximo nó.

- Transit LSR: LSRs intermediários têm a função de apenas trocar o rótulo (swap) e encaminhar o datagrama para o próximo nó. Este é o processo que mais contribui para o ganho de desempenho na utilização do protocolo MPLS, pois os LSRs intermediários não precisam mais analisar cabeçalhos da camada de rede.

- Egress LSR: é o último nó de um caminho MPLS, e efetua a retirada do cabeçalho MPLS do datagrama, encaminhando-o segundo as informações do cabeçalho de rede.

Os LSRs de entrada e de saída também são conhecidos como $L E R$, pelo fato de se localizarem nas periferias da rede MPLS.

Um LSR é chamado upstream LSR quando ele envia os dados, e downstream $L S R$ quando ele os recebe.

Existe uma técnica conhecida como Penultimate Hop Popping [29] que consiste na configuração da retirada do cabeçalho MPLS no penúltimo LSR, e não 
no egress LSR. Uma vez encaminhado para o egress LSR, o cabeçalho MPLS não tem mais utilidade, pois será removido e encaminhado segundo informações do cabeçalho de rede ou do próximo cabeçalho MPLS.

Essa prática não compromete o funcionamento de um LSP, e propicia um ganho de desempenho nos roteadores de borda. Considerando-se o caso em que a retirada do cabeçalho MPLS é feita no egress LSR, a seqüência de eventos que ocorre nesse LSR é a seguinte:

1. Recepção do datagrama rotulado;

2. Análise do valor do rótulo;

3. Identificação como egress LSR para esse LSP;

4. Retirada do cabeçalho;

5. Análise do próximo cabeçalho MPLS (no caso de mais um nível de LSP) ou do cabeçalho de rede;

6. Encaminhamento do datagrama (rotulado ou não).

Podemos perceber que ocorrem duas análises de cabeçalho (em 2 e 5). Se o penúltimo LSR fizer um POP em vez de um SWAP, a seqüência de eventos no egress LSR seria a seguinte:

1. Recepção do datagrama rotulado ou datagrama de rede nativo, sem o cabeçalho MPLS;

2. Se for um datagrama rotulado, análise do valor do rótulo e retirada do cabeçalho MPLS; se for um datagrama de rede sem cabeçalho MPLS, análise das informações do cabeçalho de rede;

3. Encaminhamento do datagrama (rotulado ou não).

Com a utilização do penultimate hop popping, apenas uma análise de cabeçalho é realizada (2), aliviando a carga de processamento no egress LER. 
Next Hop Label Forwarding Entry (NHLFE): é uma estrutura de dados implementada em cada LSR utilizada para decidir como encaminhar datagramas com cabeçalho MPLS. As principais informações dessa estrutura são o próximo nó para o encaminhamento do datagrama e a operação sobre o cabeçalho MPLS. As operações possíveis são troca, retirada ou troca seguida de adição de um novo cabeçalho.

Incoming Label Map (ILM): contém um mapeamento para cada valor de rótulo dos datagramas que chegam em um LSR para a sua respectiva entrada na tabela de NHLFEs. Se um LSR receber um datagrama rotulado com um valor inválido, ele deve descartar esse datagrama, a menos que encaminhá-lo sem o rótulo não cause problemas (loop, por exemplo) à rede.

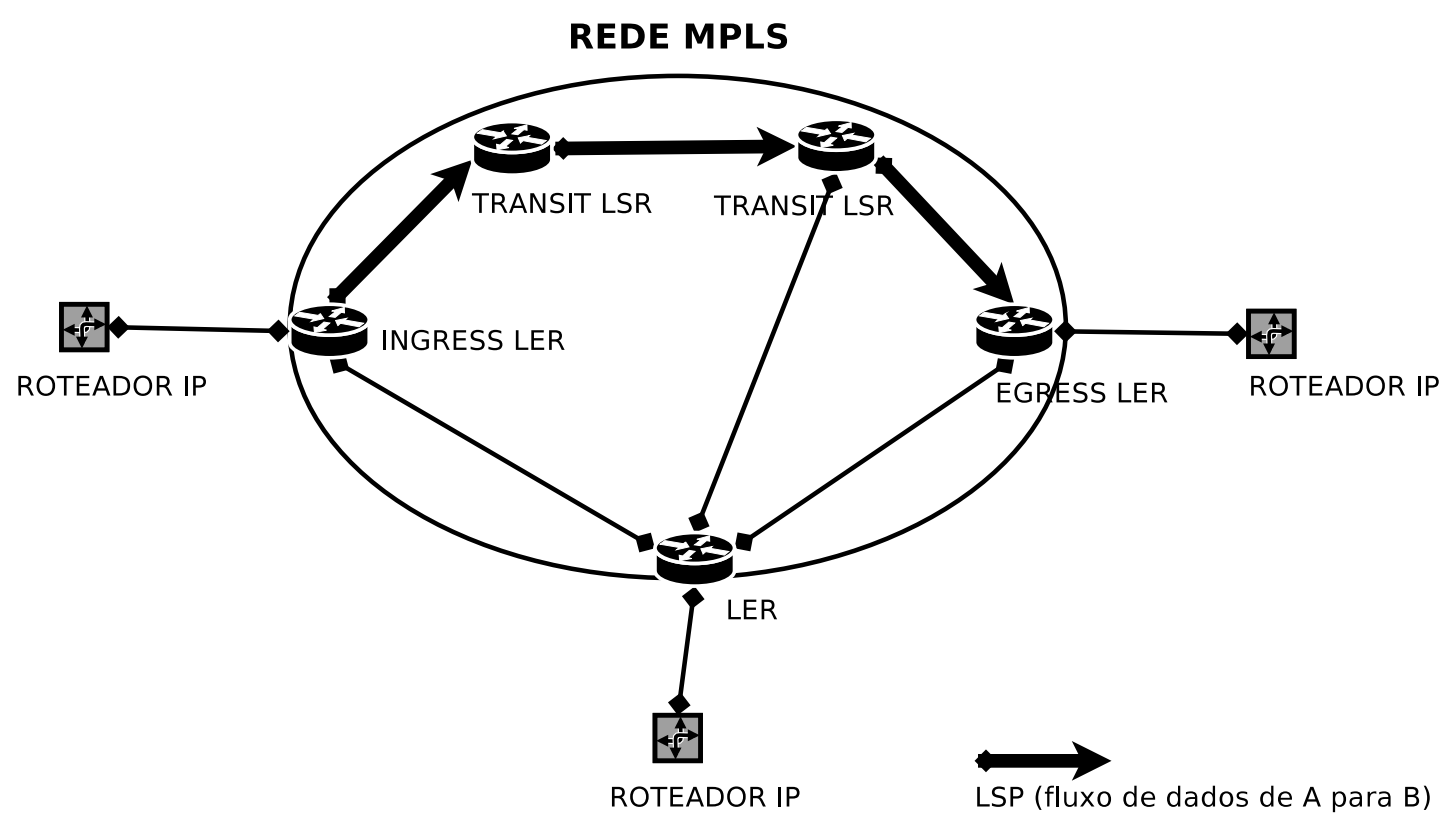

Figura 3.1: Componentes de uma rede MPLS

\subsection{Criação e manutenção de LSPs}

A criação de um LSP nada mais é do que uma atribuição e manutenção coordenada de rótulos ao longo de um conjunto contínuo de LSRs, de maneira que 
o datagrama seja forçado a seguir um caminho pré-determinado. A seguir são apresentadas algumas classificações para os LSPs [29]:

Modos de criação de LSPs: os LSPs podem ser criados de duas maneiras: LSPs Estáticos, que são configurados manualmente em cada LSR, sem envolver sinalizações (troca de informações entre os LSRs), e LSPs Sinalizados, que são configurados através de um protocolo de sinalização que gerencia a distribuição de rótulos entre os LSRs. No MPLS, a atribuição dos rótulos à FEC é feita pelo LSR downstream, que informa ao LSR upstream a associação entre um determinado rótulo e a sua respectiva FEC. Ao receber o rótulo para a associação, cada LSR deve verificar se esse valor está disponível e dentro do intervalo de valores suportados. Como exemplos de protocolos de sinalização podemos citar Label Distribuition Protocol (LDP) [34] e RSVP [22].

Modos de Atribuição de Rótulos: existem duas formas de se associar rótulos a uma FEC. Em uma delas, a associação é feita previamente, antes de qualquer tráfego de datagramas com as características atribuídas a essa FEC. Esse tipo de atribuição é classificada como orientada a controle. Na outra, a atribuição é feita à medida que esses datagramas entram na rede MPLS, e é conhecida como orientada a tráfego. Os LSRs podem criar uma associação logo no primeiro datagrama ou esperar um determinado número de datagramas para fazê-lo. Quando a criação de LSPs segue o modelo orientado a tráfego, é necessário que os LSRs sejam capazes de encaminhar datagramas através de roteamento nível 3, para não interromper o funcionamento da rede.

Modos de Distribuição de Rótulos: há dois métodos de distribuição de rótulos: Downstream-on-demand e Unsolicited downstream. No modo downstreamon-demand, cada LSR solicita um rótulo para uma FEC ao seu LSR seguinte. No modo unsolicited downstream, um LSR distribui associações de rótulos para seus vizinhos sem que eles tenham solicitado tal informação. 
Modos de Retenção de Rótulos: existem dois tipos de procedimentos em relação a retenção de rótulos: liberal e conservador. No modo liberal, um LSR salva qualquer associação rótulo - FEC A recebida, mesmo que seja de um LSR que não seja seu próximo nó para a FEC A. Esta estratégia possibilita que a construção de LSPs seja mais rápida, mas exige mais recursos dos LSRs. No modo conservador, o LSR aceita apenas associações rótulo - FEC A de um LSR que seja seu próximo nó para a FEC A. Esta estratégia exige menos recursos, mas a efetivação das alterações é mais lenta.

\subsection{Cabeçalho}

Um cabeçalho MPLS pode ser encapsulado em vários protocolos de nível 2 e pode encapsular qualquer protocolo de nível 3 [35]. Alguns deles já possuem campos que podem ser utilizados para transportar o rótulo MPLS (como exemplo, podemos citar os campos Virtual Path Identifier (VPI) e Virtual Channel Identifier (VCI) no cabeçalho ATM [36]). Em protocolos que não possuem esses campos, tais como Ethernet e Point-to-Point Protocol (PPP), o cabeąlho MPLS é inserido entre os cabeçalhos de nível 2 e nível 3.

O cabeçalho MPLS tem 32 bits, divididos nos seguintes campos (figura 3.2):

\begin{tabular}{|l|l|l|l|l|}
\hline 0 & 20 & 23 & 24 & 31 \\
\hline LABEL & $\underline{\text { EXP }}$ & $\underline{\mathrm{S}}$ & $\underline{\underline{T L}}$ & $\underline{\text { DATAGRAMA IP }}$ \\
\hline
\end{tabular}

Figura 3.2: Cabeçalho MPLS

Rótulo: este campo (LABEL) contém o valor do rótulo. Seu valor só tem significado entre os dois LSRs vizinhos, pois ele é trocado por um outro valor no próximo nó. Como o tamanho é de 20 bits, esse valor pode variar de 0 a $2^{(20-1)}$. Existem alguns valores que são reservados ao protocolo, e têm significados especiais [35]: 
- 0 - IPv4 Explicit NULL Label: indica que o rótulo deve ser retirado, e desse ponto em diante, o roteamento será feito com base no endereço de rede.

- 1 - Router Alert Label: indica que o datagrama deve ser analisado pelo software local. O encaminhamento seguinte é definido pelo próximo rótulo da pilha MPLS.

- 2 - IPv6 Explicit NULL Label: mesma funcionalidade do valor 0, mas aplicada ao protocolo IPv6.

- 3 - Implicit NULL Label: valor utilizado pelos LSRs para a distribuição de rótulos (LDP).

- 4 a 15: Reservados para definições futuras.

Bits Experimentais: este campo (também conhecido como EXP) é composto por três bits reservados para estudo futuro. Estão sendo desenvolvidos estudos para definir um padrão na utilização desse campo para o controle de serviços diferenciados (DiffServ) [37].

Stack Bit: o campo Stack Bit (S) é formado por apenas um bit, e indica se o cabeçalho ao qual ele pertence é o último da pilha MPLS (mais próximo ao cabeçalho de nível 3). Todos os outros cabeçalhos MPLS devem ter esse bit em 0 . Através deste campo, um LER de saída tem condições de decidir se o próximo encaminhamento será baseado em MPLS ou IP (rede).

Time To Live: o campo TTL é formado por oito bits, e funciona de maneira semelhante ao TTL do protocolo IP (correção de loop). Se o destino do datagrama não for o nó local e o TTL for zero, esse datagrama pode ser simplesmente descartado ou ativar a geração de uma mensagem ICMP. Quando um datagrama entra em um LSP, o valor inicial do TTL no cabeçalho MPLS deve ser igual ao valor do TTL do cabeçalho IP, e decrementado de 1 em cada LSR. Na saída do 
LSP, o egress LER deve copiar o valor do TTL do cabeçalho MPLS para o TTL do cabeçalho IP. Como o cabeçalho IP somente é alterado na saída de um domínio MPLS, o checksum não é realizado dentro desse domínio. Alguns protocolos de nível 2 (notavelmente o ATM), não possuem o campo TTL, e o tratamento de loops deve ser feito de outra maneira. Trechos onde os LSRs não são capazes de utilizar o campo TTL são conhecidos como non-TTL LSR segment. Nesses casos, o ingress LSR deve ser capaz de saber de quantos nós é constituído esse segmento, para modificar o TTL do cabeçalho IP antes de encaminhar o datagrama.

Como o nome diz, MPLS é multiprotocolo, ou seja, é compatível com vários protocolos das camadas 2 e 3. A figura 3.3 ilustra três exemplos de inserção do protocolo MPLS em uma arquitetura de rede.

A figura 3.3a mostra a utilização do MPLS em redes ATM. Nesse tipo de rede, deve haver um cabeçalho MPLS antes do cabeçalho IP. O valor do rótulo desse cabeçalho é armazenado nos campos VPI e VCI. O valor do rótulo no cabeçalho MPLS deve ser zero e ignorado pelos LSRs durante a transmissão, considerando apenas o valor em VPI/VCI. Os valores dos campos EXP, S e TTL devem ser obtidos do primeiro cabeçalho da pilha MPLS.

Nos casos em que não há um campo específico para o valor do rótulo, o cabeçalho MPLS deve ser inserido entre os cabeçalhos das camadas 2 e 3 . Como exemplo de tais protocolos, temos o Ethernet (figura 3.3b) e PPP (figura 3.3c).

No transporte de datagramas rotulados pelo MPLS, o protocolo PPP atribui o valor hexadecimal 8281 ao campo "protocolo" do seu cabeçalho, enquanto que no Ethernet, o campo "tipo" pode receber os valores hexadecimais 8847 (MPLS Unicast) ou 8848 (MPLS Multicast [38]).

Uma característica importante do MPLS é a definição de pilha de rótulos, que consiste em uma seqüência de cabeçalhos MPLS. Isso significa que um datagrama rotulado pode conter na sua área de dados outro datagrama rotulado. Essa característica possibilita a criação de LSPs hierárquicos, ou seja, LSPs sobre outra LSP. Em redes Ethernet, redes MPLS hierárquicas são criadas adicionando-se 


\section{ATM}

a)

\begin{tabular}{|l|l|l|l|l|l|l|}
\hline GFC & $\underline{\mathrm{VCI}}$ & $\underline{\mathrm{VPI}}$ & $\underline{\mathrm{PTI}}$ & $\underline{\mathrm{CLP}}$ & $\underline{\mathrm{HEC}}$ & $\underline{\text { DATA }}$ \\
\hline
\end{tabular}

ETHERNET

b)

\begin{tabular}{|l|l|l|l|}
\hline MAC & MPLS & $\underline{\text { IP }}$ & DATA \\
\hline
\end{tabular}

PPP

c)

\begin{tabular}{|l|l|l|l|}
\hline$\underline{P P P}$ & $\underline{M P L S}$ & $\underline{\text { IP }}$ & DATA \\
\hline
\end{tabular}

Figura 3.3: Cabeçalho MPLS em diferentes protocolos da camada 2 uma seqüência de cabeçalhos MPLS (figura 3.4).

\section{ETHERNET}

\begin{tabular}{|l|l|l|l|l|}
\hline ETHERNET & MPLS & MPLS & $\underline{\text { IP }}$ & DATA \\
\hline
\end{tabular}

Figura 3.4: Hierarquia: 2 níveis de profundidade

No caso de redes MPLS hierárquicas em redes ATM, todos os cabeçalhos MPLS devem ser inseridos antes do cabeçalho IP, e apenas o valor do rótulo do primeiro cabeçalho MPLS (topo) deve ser copiado para VPI/VCI.

\subsection{Fragmentação}

O processo de tratamento de datagramas em relação à fragmentação tem um papel importante no roteamento em redes MPLS. É preciso manter as funcionalidades oferecidas pela fragmentação de datagramas IP [12]. As regras para o tratamento de fragmentação no MPLS são as seguinte:

Para datagramas sem cabeçalho MPLS: 
1. Cada LSR tem um parâmetro conhecido como Maximum Initially Labeled IP Datagram Size, ao qual pode ser atribuído qualquer valor não-negativo. Esse parâmetro determina o tamanho máximo de um datagrama que vai entrar em um LSP;

2. Quando um LSR recebe datagramas IP sem rótulo MPLS, o LSR verifica o tamanho desse datagrama;

3. Se o datagrama for menor que o valor do parâmetro acima, ele deve receber o cabeçalho MPLS e ser encaminhado normalmente;

4. Se o datagrama for maior que o valor do parâmetro acima e seu bit DF não estiver ativado (valer 0), ele deve ser divido em fragmentos de tamanho igual ou menor que o valor desse parâmetro, receber o cabeçalho MPLS e ser encaminhado.

\section{Para datagramas com cabeçalho MPLS:}

1. Se o datagrama rotulado for maior que o tamanho máximo da área de dados do frame (M) e seu bit DF não estiver ativado (valer 0), ele pode ser descartado silenciosamente;

2. Se o LSR decidir não descartar o datagrama nas condições anteriores, ele deve retirar todos os cabeçalhos MPLS do datagrama IP e dividí-lo em fragmentos de tamanho M-N, onde N é o tamanho da pilha MPLS. Em seguida, o LSR deve atribuir o mesmo rótulo para todos os fragmentos e encaminhá-los ao próximo nó;

3. Se o datagrama for maior que $M$ e seu bit DF estiver ativado (valer 1), ele deve ser descartado e uma mensagem ICMP do tipo "Fragmentação necessária e bit DF ativado" deve ser gerada e enviada à sua origem, se possível. 
Para enviar mensagens ICMP à origem do datagrama, o LSR deve ser capaz de identificar que o cabeçalho de nível 3 é um cabeçalho IP, além de rotear o datagrama com a mensagem ICMP através do endereço IP origem do datagrama.

\subsection{Qualidade de Serviço}

Assim como no protocolo IP, a Engenharia de Tráfego é um mecanismo interessante para se alcançar qualidade de serviço em uma rede que utiliza o protocolo MPLS. Segundo [39], para que uma rede MPLS suporte engenharia de tráfego, é necessário que ela ofereça três funcionalidades:

1. Definir e interpretar um conjunto de características associadas ao tráfego. Essas características definem o tratamento que deve ser dado a ele;

2. Definir e interpretar um conjunto de características associadas aos recursos. Essas características são utilizadas para controlar o tráfego injetado nos LSRs envolvidos;

3. Definir um sistema de roteamento para selecionar os caminhos que cada tipo de tráfego deve seguir, segundo os dois atributos acima.

Com o protocolo MPLS é possível distribuir o tráfego de dados entre rotas (LSPs) diferentes com informações além da camada de rede. Informações como interface de entrada (camada 2) ou portas TCP/UDP (camada 4) podem ser utilizadas para decidir como encaminhar um datagrama IP. O ingress LSR analisa essas informações e faz a associação do datagrama a uma FEC. Essa possibilidade oferece uma granularidade mais fina e, conseqüentemente, uma utilização mais racional dos recursos da rede.

Como exemplo, podemos citar uma separação de tráfegos FTP (dados), que exige largura de banda, e Secure Shell (SSH), que exige baixo atraso. A conexão FTP pode ser direcionada para um rota com um alto atraso e alta largura de 
banda, e a conexão SSH (aplicação interativa) para uma rota com baixo atraso e pequena largura de banda.

Além da engenharia de tráfego, o protocolo MPLS suporta o tratamento diferenciado para os datagramas com base no valor do campo DSCP (serviços diferenciados [37]). Neste caso, a classificação dos datagramas foi feita na camada 3 (IP), e já existe um valor adequado no campo TOS (DSCP). Para que um domínio MPLS continue suprindo as necessidades de recurso que foram definidas, é necessário que haja um mapeamento das classes de serviço representadas pelo DSCP nas FECs do ingress LSR de um LSP.

O PHB que deve ser aplicado ao datagrama pode ser deduzido de duas maneiras: a primeira é através do campo EXP do cabeçalho MPLS. Nesse caso, tanto a prioridade de processamento como a probabilidade de descarte do datagrama rotulado estarão representados nesse campo. LSPs com esse tipo de comportamento suportam vários classes de tratamento de datagramas, pois mesmo existindo apenas um rótulo para o LSR decidir o encaminhamento, podem existir vários valores referentes a PHBs no campo EXP.

A segunda é através do rótulo. Neste caso, a prioridade de processamento de um datagrama é obtida do valor do rótulo e a sua probabilidade de descarte, obtida do campo EXP. Este tipo de LSP suporta apenas uma classe de prioridade de processamento, já que o valor do rótulo em um LSR é sempre o mesmo para um LSP. Quando não houver cabeçalho MPLS (ATM, por exemplo), a probabilidade de descarte é obtida de campos específicos da camada 2, os quais já realizam essa função. 


\section{Capítulo 4}

\section{Breve comparação entre redes IP e}

\section{redes IP sobre MPLS}

O surgimento de novas tecnologias normalmente ocorre porque a tecnologia atual não atende às necessidades, sejam elas funcionais ou por desempenho. O MPLS surgiu como um protocolo que oferece algumas funcionalidades não existentes em redes IP convencionais. Este capítulo apresenta as principais diferenças entre as duas arquiteturas e suas conseqüências nos processos de roteamento e encaminhamento de datagramas.

\subsection{Modelo OSI}

O primeiro item a ser discutido é o posicionamento no modelo OSI de camadas. Redes IP que trafegam sobre quadros Ethernet são claramente definidas como camada 3 sobre camada 2 . Já redes IP encapsuladas em MPLS e trafegando sobre Ethernet, violam o modelo OSI, e têm o protocolo MPLS como uma ligação entre as camadas 2 e 3, e algumas referências o classificam como camada 2,5 [33]. 


\subsection{Formato de cabeçalho}

Em redes IP, para decidir como encaminhar os datagramas, os roteadores utilizam informações do cabeçalho IP. Este cabeçalho possui uma estrutura complexa, sendo necessária a análise de vários campos para realizar o roteamento, além de conhecer os limites e tamanho de cada um. Já em uma rede IP sobre MPLS, as informações necessárias para o encaminhamento são obtidas do cabeçalho MPLS, que é bem menor e menos complexo que o cabeçalho IP.

Esses dois fatores fazem com que equipamentos de menor poder de processamento e armazenamento (buffers) tenham desempenho melhor em arquiteturas IP sobre MPLS do que em arquiteturas IP diretamente sobre Ethernet.

\subsection{Endereçamento}

No protocolo MPLS, os roteadores não possuem em endereçamento identificador (algo como o endereço IP, por exemplo). A identificação dos LSRs é feita pelo endereço de rede. A identificação feita pelo protocolo MPLS é dos LSPs, ou seja, as FECs são identificadas pelo rótulo.

\subsection{Encaminhamento $\mathrm{x}$ Roteamento}

Encaminhamento é um processo local a cada roteador, que consiste no recebimento de datagramas por uma interface de entrada e no envio ao próximo roteador por uma interface de saída. A escolha da interface de saída e do próximo roteador é feita baseada numa tabela de encaminhamento, presente em cada roteador. Em redes IP, essa tabela tem como índice um endereço de um nó ou de uma rede, e a busca é feita utilizando-se o endereço IP de destino dos datagramas, com o algoritmo de coincidência mais longa (longest match). Em redes IP sobre MPLS, essa tabela tem como índice um valor de rótulo, e a busca é feita utilizando-se o valor do rótulo presente no cabeçalho MPLS. 
Essa diferença de paradigma resulta em duas vantagens à arquitetura IP sobre MPLS: maior desempenho, devido ao menor tamanho e complexidade de um rótulo em relação a um endereço IP e menor complexidade nos algoritmos de encaminhamento. Para suportar as várias funcionalidades de roteamento, o protocolo IP possui diferentes algoritmos de encaminhamento. Por exemplo, o algoritmo de coincidência mais longa é necessário para dar precedência a rotas para hosts sobre rotas para redes. Quando a rede precisa realizar tratamento diferenciado aos tipos de tráfego (DiffServ), o algoritmo torna-se ainda mais complexo.

No MPLS, o algoritmo de encaminhamento é único: coincidência exata nos valores de rótulo. O ingress LER analisa o datagrama IP e atribui a ele um valor de rótulo adequado. Os LSRs seguintes utilizam apenas o algoritmo de coincidência exata, pois mesmo que haja tráfego agregado (datagramas com diferentes endereços de origem e/ou destino que seguem pelo mesmo caminho), todos os datagramas terão o mesmo valor de rótulo. Como o MPLS atribui tarefas adicionais aos LERs (atribuição de rótulos aos datagramas, por exemplo), a utilização de CPU aumenta nesses equipamentos.

Já o roteamento é um processo que abrange toda uma rede, no qual os roteadores comunicam-se entre si para criar as tabelas de encaminhamento, de maneira que os datagramas sejam enviados para os roteadores adequados e sigam a melhor rota possível. Nas redes IP convencionais, todos os roteadores precisam conhecer a melhor rota para encaminhar o datagrama pelo melhor caminho possível. O protocolo MPLS trabalha apenas no encaminhamento dos datagramas rotulados, pois os LSRs não têm acesso ao endereço IP destino desses datagramas. Dessa forma, não existe inteligência de roteamento nos LSRs intermediários, apenas o encaminhamento local, de uma interface para outra com base nos valores dos rótulos. 


\subsection{Rotas pré-determinadas}

Com o MPLS é possível determinar a rota (LSP) dos datagramas na sua origem. Através do valor do rótulo do datagrama recebido, o LSR pode determinar qual é o próximo nó, para onde o datagrama deve ser enviado. Para configurar uma rota, basta atribuir os rótulos adequadamente em cada LSR. No roteamento IP, a única maneira de se determinar a rota na origem é através do campo $I P$ OPTIONS, utilizando uma técnica conhecida como source routing. Essa técnica consiste na criação de uma lista de endereços IP que o datagrama deve seguir até alcançar o seu destino.

O source routing oferece um mecanismo para se determinar a rota no ponto de origem do datagrama, mas tem dois pontos fracos: um deles é a quantidade de endereços IP que podem ser especificados, pois o tamanho do cabeçalho é limitado pelo valor máximo possível de ser representado no campo HLEN (tamanho do cabeçalho IP); o outro é que esse mecanismo continua exigindo a pesada tarefa de se processar o cabeçalho IP.

\subsection{Relevância do ponto de entrada}

Outra propriedade interessante do MPLS é a possibilidade de se utilizar o ponto de entrada de um datagrama para decisões de encaminhamento. Os LSRs mantêm uma tabela de roteamento que é indexada pelo valor do rótulo de chegada, e esse valor está relacionado com a interface de chegada.

No roteamento IP convencional não é possível identificar por qual interface o datagrama chegou ao roteador, pois essa informação não caminha com o datagrama. 


\subsection{Escalabilidade}

A principal informação carregada por um datagrama IP que é usada para decisões de roteamento é o endereço IP destino. Para que os roteadores IP consigam encaminhar os datagramas, eles necessitam de uma rota para todos os destinos possíveis. Isso traz uma complexidade no sistema de roteamento, à medida que a quantidade de redes aumenta.

Já os rótulos têm significado local, ou seja, seu valor é importante apenas entre LSRs vizinhos. Graças a esse conceito, os LSRs tornaram-se meros "chaveadores de datagramas". Por mais complexa que seja uma rede, a única inteligência necessária nos nós intermediários é a capacidade de decidir localmente como encaminhar o datagrama, diferentemente do roteamento IP. A inteligência para elaborar uma rota adequada (LSP) está toda no ingress LER.

\subsection{Engenharia de Tráfego e Qualidade de Serviço}

As possibilidades de engenharia de tráfego com o MPLS são muito maiores do que em redes IP convencionais (IP sobre Ethernet, por exemplo). Nas redes IP, as tabelas de roteamento são indexadas pelo endereço destino do datagrama. Conseqüentemente, a menor granularidade possível é baseada em endereços IP.

Já com o MPLS, as tabelas de encaminhamento nos LSRs são indexadas pelo valor do rótulo. Como o rótulo não tem nenhuma codificação do endereço de rede, é possível associá-lo a várias informações presentes no datagrama, inclusive informações de outras camadas. Isso possibilita, por exemplo, a criação de "rotas" (LSPs) baseadas no tipo de aplicação (portas TCP ou UDP), resultando numa granularidade mais fina para realizar o roteamento e, conseqüentemente, uma utilização mais racional e eficiente dos recursos de rede.

No protocolo IP, o tratamento diferenciado de datagramas é feito através da atribuição de diferentes valores ao campo TOS do cabeçalho. A influência desse 
campo no roteamento IP limita-se aos seguintes casos:

- Existência de duas ou mais rotas para um mesmo destino: o roteador pode utilizar o DSCP para decidir qual delas utilizar;

- Os roteadores podem implementar internamente várias diferentes filas de datagramas, com cada uma delas recebendo um tipo de tratamento. O roteador escolhe para qual fila encaminhar o datagrama de acordo com o valor do DSCP.

Podemos perceber que a decisão de qual rota seguir é determinada pelo endereço IP de destino.

Utilizando-se o protocolo MPLS, além de oferecer uma compatibilidade em relação ao comportamento dos roteadores, é possível criar LSPs dedicados para uma determinada classe de serviço. Por exemplo, o ingress LSR pode analisar o DSCP do cabeçalho IP e direcionar tráfegos da classe AF para um LSP e da classe EF para outro. Isso aumenta ainda mais as possibilidades de engenharia de tráfego em uma rede IP sobre MPLS. 


\section{Capítulo 5}

\section{Análise de desempenho}

O objetivo principal deste trabalho é fornecer dados que caracterizem o desempenho das duas arquiteturas envolvidas (roteamento IP e roteamento IP sobre MPLS) e realizar uma análise comparativa entre elas. Para a execução deste trabalho foram construídos vários tipos de rede que permitiram avaliar o comportamento das duas arquiteturas em diferentes situações.

Para garantir a fidelidade dos resultados e, conseqüentemente, das conclusões, foi preciso tomar uma série de cuidados e evitar a interferência de fatores externos ao contexto analisado.

O mesmo hardware foi utilizado tanto nas medições de roteamento IP como nas medições de roteamento IP sobre MPLS. Para que os resultados reflitam apenas o desempenho da arquitetura de protocolos, a única diferença entre os sistemas operacionais das duas arquiteturas é o suporte ao protocolo MPLS, já que otimizações diferentes poderiam interferir nos resultados. Metodologias que envolvem acesso a disco podem fornecer resultados alterados, pois esse acesso a disco pode limitar a capacidade de transferência de um roteador. E finalmente, determinadas aplicações podem utilizar técnicas que afetem o desempenho de uma transmissão (compactação, por exemplo). Em [40] pode ser encontrada uma documentação detalhada sobre avaliação de equipamentos de rede.

Levantadas essas questões, a metodologia escolhida para a execução dos testes 
foi a geração de tráfego através do netperf [41]. Ela gera os dados na memória principal para realizar a medição, ao invés de ler e enviar um arquivo. O tráfego de dados pode ser através dos protocolos TCP ou UDP. Os roteadores das redes de testes são PCs com o sistema operacional Linux [42] configurados com o kernel 2.6.9 (tabela 5.1).

Tabela 5.1: Descrição dos equipamentos utilizados

\begin{tabular}{|l|l|}
\hline Nome & Características \\
\hline HOSTA & $\begin{array}{l}\text { Sempron 1.6GHz 512MB RAM com 1 placas Realtek 8139 10/100 } \\
\text { PCI, } \\
1 \text { placa SIS900 10/100 PCI e 1 placa RealTek 8110S Gb Ethernet } \\
\text { PCI }\end{array}$ \\
\hline HOSTB & $\begin{array}{l}\text { Athlon 64 3000 1GB RAM com uma placa nForce 1Gb PCI e uma } \\
\text { RealTek 8169 1Gb PCI }\end{array}$ \\
\hline LSR1 & $\begin{array}{l}\text { Sempron 1.6GHz 512MB RAM com 3 placas Realtek 8139 PCI e 1 } \\
\text { placa SIS900 PCI }\end{array}$ \\
\hline LSR2 & $\begin{array}{l}\text { Pentium 90MHz 16MB com 2 placas Realtek 8139 PCI e 1 placa } \\
\text { NE2000 ISA }\end{array}$ \\
\hline LSR3 & $\begin{array}{l}\text { Pentium 100MHz 24MB com 2 placas Realtek 8139 PCI e 1 placa } \\
\text { NE2000 ISA }\end{array}$ \\
\hline LSR4 & $\begin{array}{l}\text { Pentium 100MHz 16MB com 2 placas Realtek 8139 PCI e 1 placa } \\
\text { NE2000 ISA }\end{array}$ \\
\hline LSR5 & $\begin{array}{l}\text { Pentium 100MHz 32MB com 3 placas Realtek 8139 PCI e 1 placa } \\
\text { NE2000 ISA }\end{array}$ \\
\hline LSR6 & $\begin{array}{l}\text { Pentium II 233MHz 512MB com 3 placas Realtek 8139 PCI e 1 } \\
\text { placa NE2000 ISA }\end{array}$ \\
\hline
\end{tabular}

Para facilitar a memorização, o endereçamento dos nós nas redes lineares (sem bifurcações) seguiu os seguintes padrões:

- A primeira interface de rede ethernet dos roteadores (eth0) sempre pertence à rede de controle $(172.28 .0 .0 / 24)$;

- HOSTA estará posicionado sempre na extremidade da esquerda da rede, com o endereço IP 192.168.28.1;

- Nos roteadores representados nas figuras, eth1 estará sempre à esquerda e eth2 sempre à direita; 
- As redes IP possuem máscara 27 e estão em seqüência, da esquerda para a direita (192.168.28.0/27, 192.168.28.32/27 e assim por diante).

Os testes realizados analisam 4 aspectos relacionados ao roteamento IP e roteamento IP sobre MPLS:

- Processamento de cabeçalho;

- Quantidade de nós intermediários;

- Posicionamento dos roteadores segundo a capacidade de processamento;

- Otimização de uso dos recursos (engenharia de tráfego).

\subsection{Avaliação inicial dos roteadores}

Antes do início dos testes, cada roteador foi submetido individualmente a um tráfego IP entre dois computadores mais potentes, para se medir a capacidade máxima de cada um (figura 5.1). Esta avaliação inicial é muito importante, pois identifica qual roteador pode estar limitando o tráfego nas redes de testes.

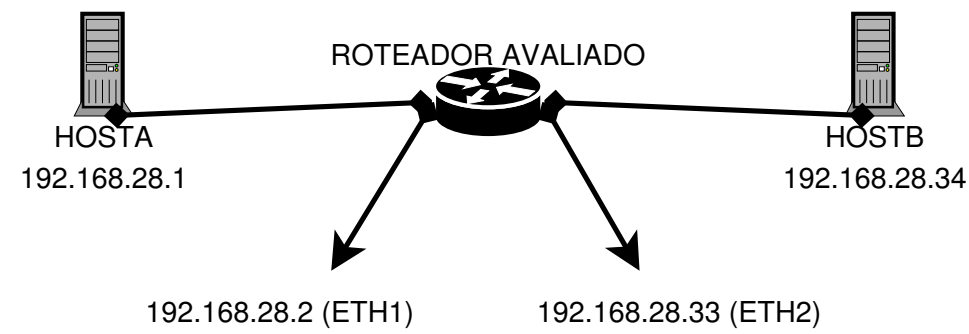

Figura 5.1: Avaliação individual dos roteadores

Nesta avaliação, HOSTA recebeu o endereço IP 192.168.28.1 e foi configurado para utilizar o endereço 192.168.28.2 como roteador para a rede de HOSTB (192.168.28.32/27), e HOSTB recebeu o endereço 192.168.28.34 e foi configurado para utilizar o endereço 192.168.28.33 como roteador para a rede de HOSTA (192.168.28.0/27): 


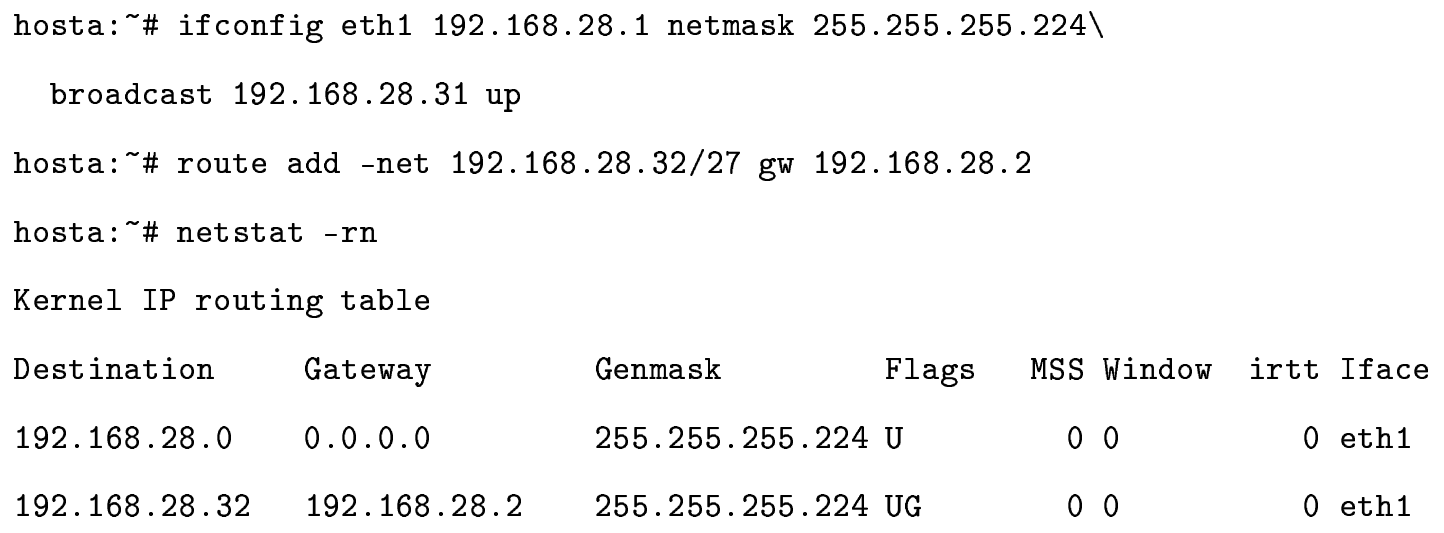

Cada roteador submetido a essa avaliação inicial teve três interfaces de rede configuradas da seguinte maneira: eth0 com um endereço IP da rede de controle, eth1 com o endereço IP 192.168.28.2 e eth2 com o endereço IP 192.168.28.33. A capacidade de roteamento nos equipamentos avaliados foi ativada através do comando sysctl:

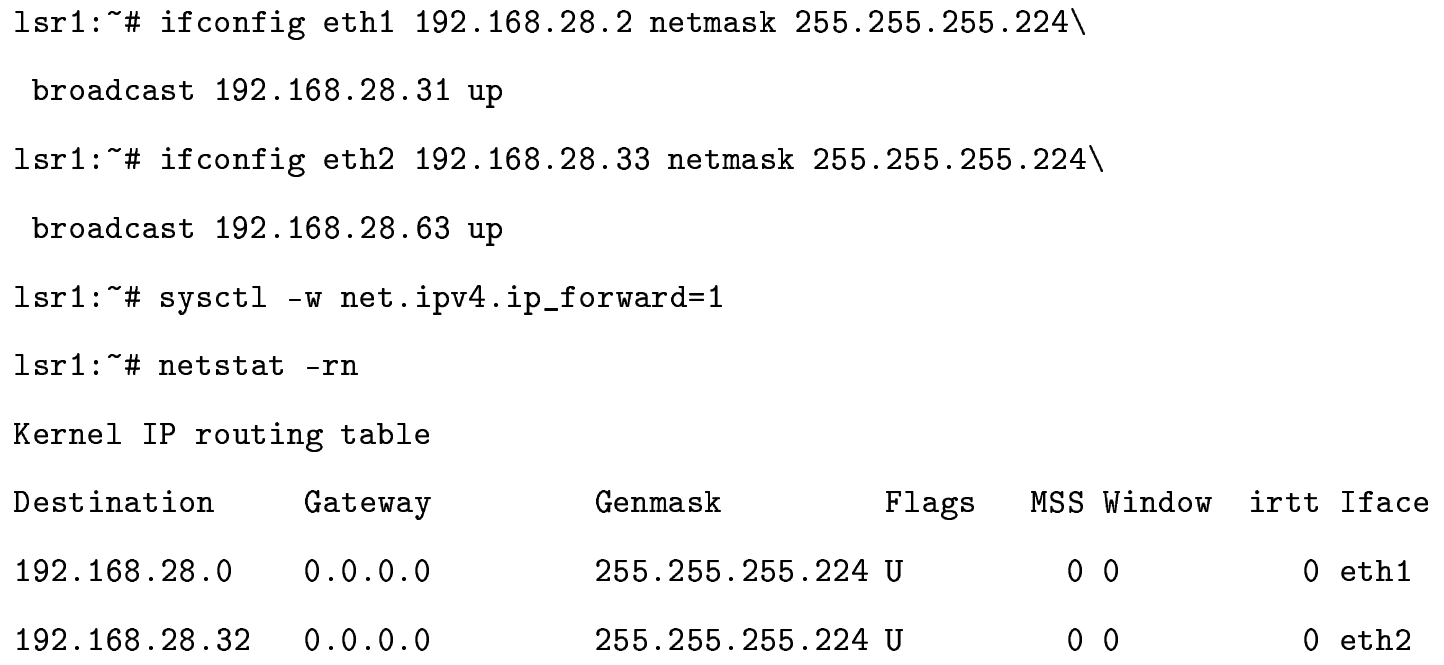


Para confirmarmos que o tráfego de dados entre HOSTA e HOSTB passa pelo roteador analisado, foi disparado um ping de HOSTA para HOSTB, e as interfaces de rede envolvidas foram monitoradas com a ferramenta tcpdump. Os relatórios gerados pelo tcpdump confirmam a passagem dos datagramas IP pelo roteador avaliado:

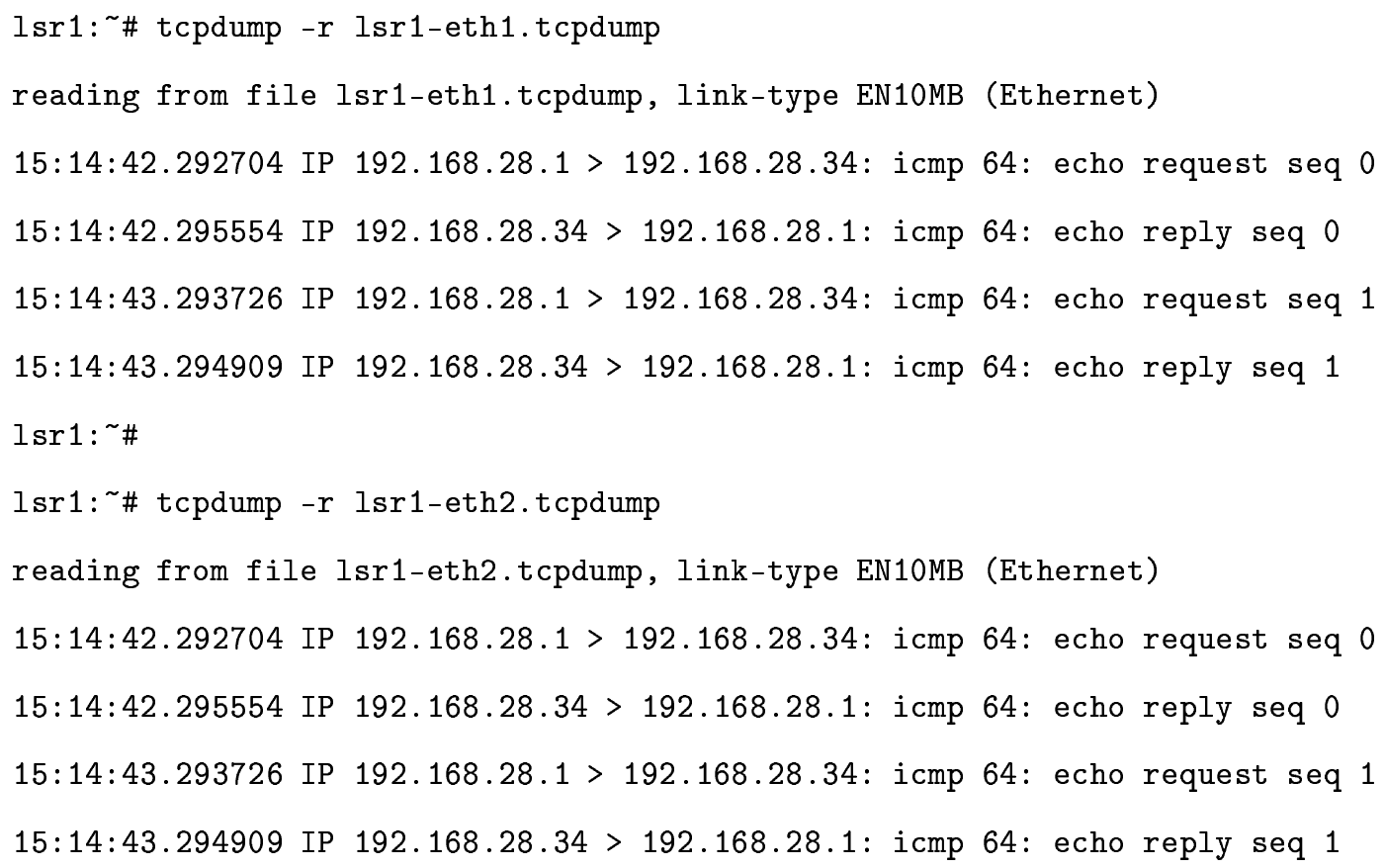

Com a rede configurada e em funcionamento, foi iniciada a geração de tráfego TCP com o netperf a partir de HOSTA em direção a HOSTB. Foram realizadas 10 medições, com uma duração de 10 segundos para cada uma delas, e obtida a média para se chegar ao valor final. O tempo de 10 segundos foi escolhido porque a diferença entre os resultados na execução nesse intervalo e em um intervalo de 24 horas foi relativamente pequena (algumas dezenas de Kbps).

hosta: \#/usr/local/netperf/bin/netperf -t TCP_STREAM -H 192.168.28.34 -1 10

Os resultados estão ilustrados na figura 5.2. Apesar das unidades serem diferentes (\% e Mbps), as duas medidas são apresentadas no mesmo gráfico, para facilitar a visualização do relacionamento entre as mesmas.

Com essas informações é possível trabalhar o equipamento menos potente e observar os ganhos (ou perdas) de desempenho decorrentes da utilização das duas 


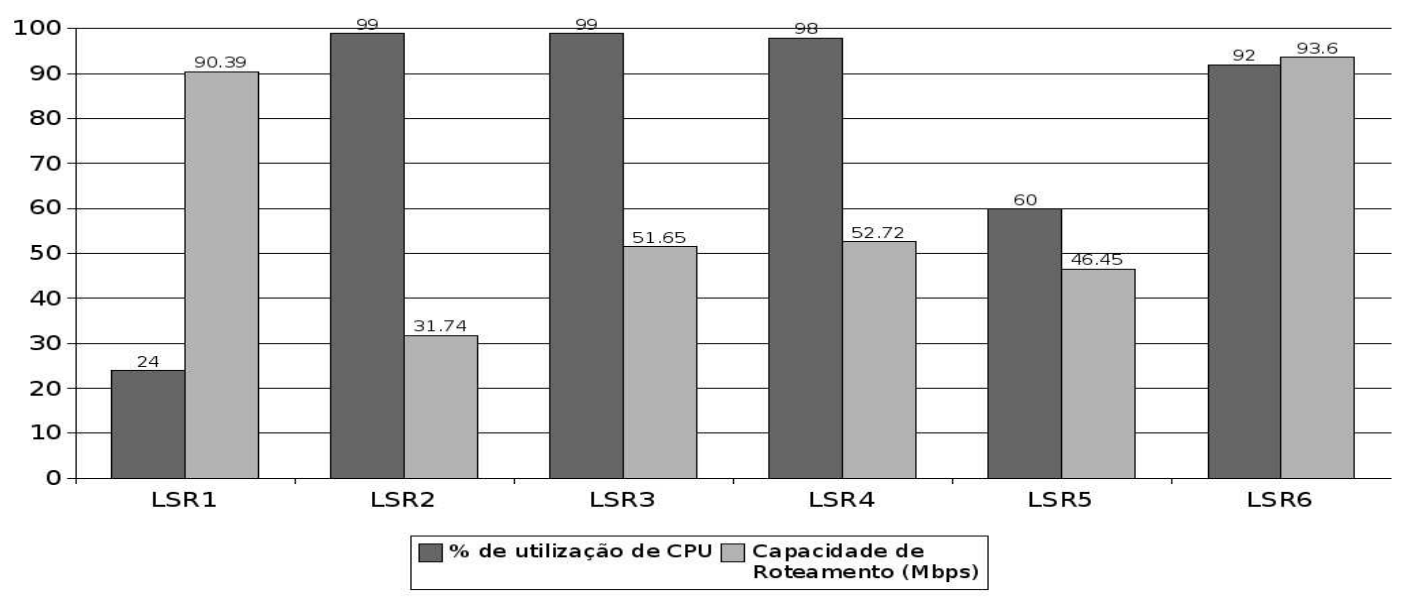

Figura 5.2: Capacidade individual de roteamento IP

arquiteturas em questão. O ajuste de roteadores que não estão operando no limite do seu poder de processamento não tem efeito na rede como um todo, pois o nó mais fraco continuará limitando o tráfego.

Segundo a figura 5.2, LSR2 é o equipamento de menor capacidade de roteamento, e será o fator limitante no tráfego das redes em que fizer parte.

\subsection{Processamento de cabeçalho e encapsulamento}

Este cenário avalia o impacto causado pela adição do protocolo MPLS à arquitetura TCP/IP. A rede construída para este teste está ilustrada pela figura 5.3.

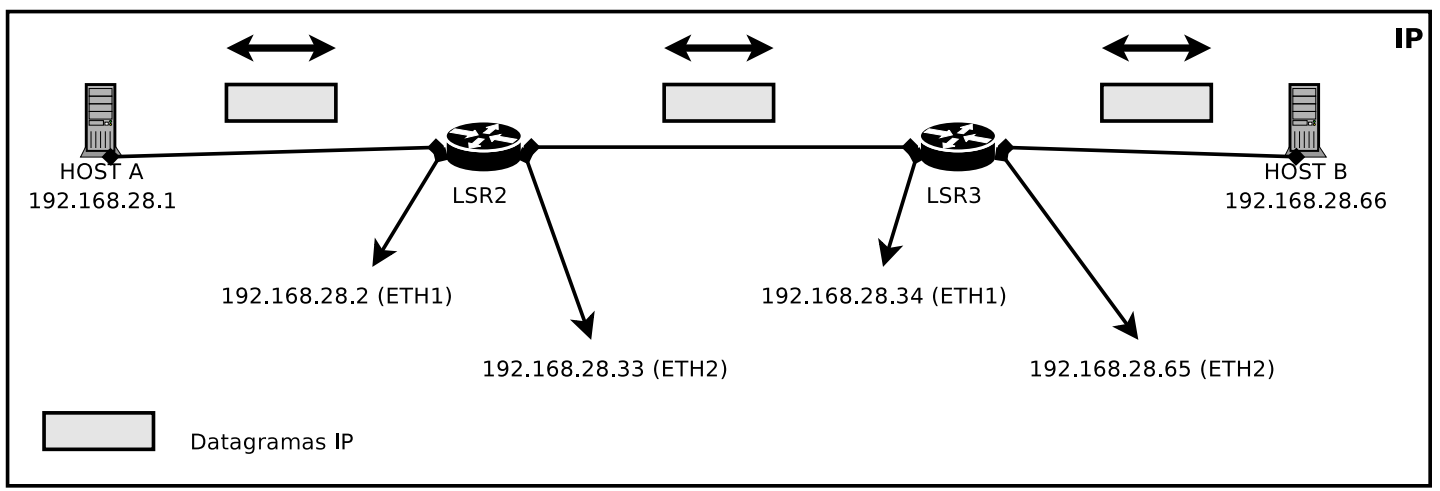

Figura 5.3: Dois roteadores IP

A primeira arquitetura testada foi o roteamento IP. HOSTA continuou com o endereço IP 192.168.28.1 na sua interface de rede eth1 e com o endereço 
192.168.28.2 (LSR2) como roteador para a rede 192.168.28.64/27. HOSTB recebeu o endereço 192.168.28.66 na sua interface de rede eth1 e foi configurado para utilizar 192.168.28.65 (LSR3) como roteador para a rede 192.168.28.0/27.

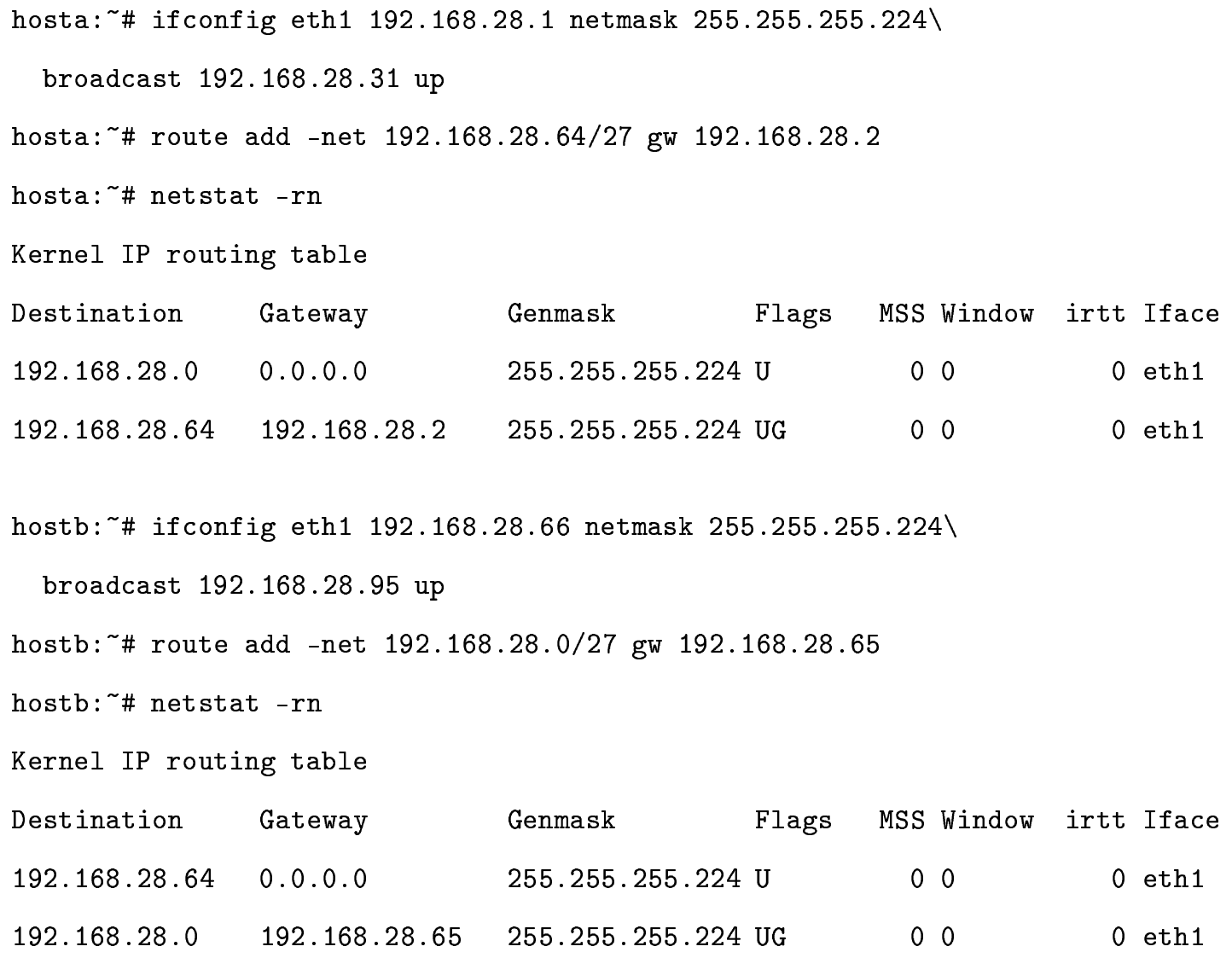

Nesta situação, ambos os roteadores (LSR2 e LSR3) estão utilizando um kernel sem suporte ao protocolo MPLS. LSR2 precisa de uma rota para a rede 192.168.28.64/27 através de 192.168.28.34 (LSR3) e LSR3 precisa de uma rota para a rede 192.168.28.0/27 através de 192.168.28.33 (LSR2).

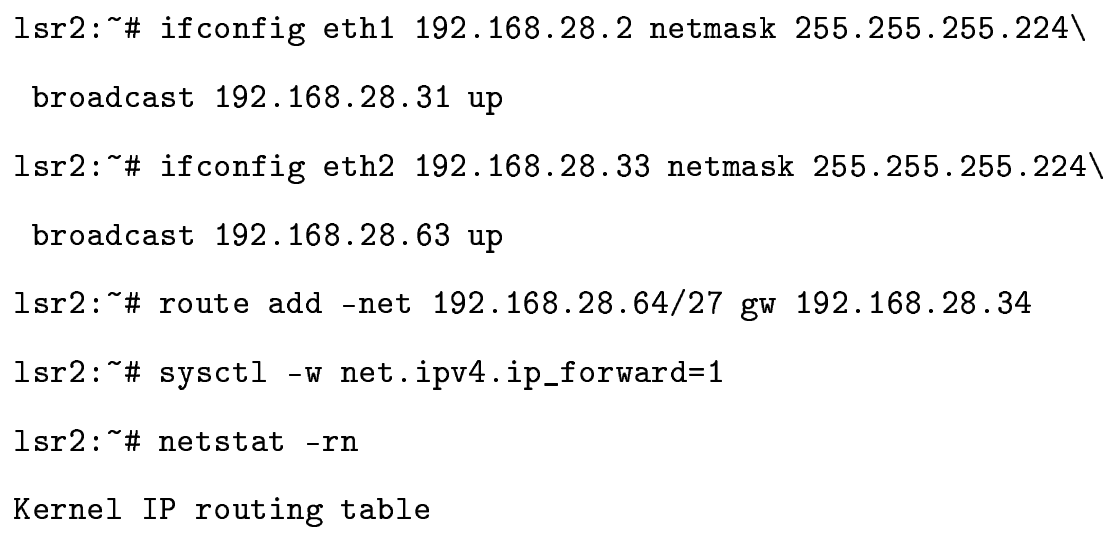




\begin{tabular}{|c|c|c|c|c|c|c|c|}
\hline Destination & Gateway & Genmask & Flags & MSS & Window & irtt & If ace \\
\hline 172.28 .0 .0 & 0.0 .0 .0 & 255.255 .0 .0 & $\mathrm{U}$ & 0 & 0 & 0 & eth0 \\
\hline 192.168 .28 .0 & 0.0 .0 .0 & 255.255 .255 .224 & $\mathrm{U}$ & 0 & 0 & 0 & eth1 \\
\hline 192.168 .28 .32 & 0.0 .0 .0 & 255.255 .255 .224 & U & 0 & 0 & 0 & eth2 \\
\hline 192.168 .28 .64 & 192.168 .28 .34 & 255.255 .255 .224 & UG & 0 & 0 & 0 & eth2 \\
\hline \multicolumn{8}{|c|}{$\begin{array}{l}\text { lsr3: \# ifconfig eth1 } 192.16 \\
\text { broadcast } 192.168 .28 .63 \text { up }\end{array}$} \\
\hline \multicolumn{8}{|c|}{$\begin{array}{l}\text { lsr3: \# ifconfig eth2 } 192.168 \\
\text { broadcast } 192.168 .28 .95 \text { up }\end{array}$} \\
\hline \multicolumn{8}{|c|}{ 1sr3: \# route add -net $192.168 .28 .0 / 27$ gw 192.168 .28 .33} \\
\hline \multicolumn{8}{|c|}{ 1sr3: \# netstat -rn } \\
\hline \multicolumn{8}{|c|}{ Kernel IP routing table } \\
\hline Destination & Gateway & Genmask & Flags & MSS & Window & irtt & If ace \\
\hline 172.28 .0 .0 & 0.0 .0 .0 & 255.255 .0 .0 & $\mathrm{U}$ & 0 & 0 & 0 & etho \\
\hline 192.168 .28 .0 & 192.168 .28 .33 & 255.255 .255 .224 & UG & 0 & 0 & 0 & eth1 \\
\hline 192.168 .28 .32 & 0.0 .0 .0 & 255.255 .255 .224 & $\mathrm{U}$ & 0 & 0 & 0 & eth1 \\
\hline 192.168 .28 .64 & 0.0 .0 .0 & 255.255 .255 .224 & $\mathrm{U}$ & 0 & 0 & 0 & eth2 \\
\hline
\end{tabular}

Para não haver dúvidas quanto ao caminho percorrido pelos datagramas IP, antes da medição de desempenho foram disparadas mensagens ICMP (ping) a partir do HOSTA para o HOSTB, e as interfaces de rede dos roteadores foram monitoradas com tcpdump.

Após a configuração da rede IP, a ferramenta netperf foi utilizada para gerar tráfego TCP a partir de HOSTA em direção a HOSTB. Os resultados para o roteamento IP estão na tabela 5.2.

Tabela 5.2: Roteamento IP com dois nós intermediários sem MPLS

\begin{tabular}{|r|r|r|}
\hline \%CPU LSR2 & \%CPU LSR3 & Capacidade de roteamento (Mbps) \\
\hline 99 & 63 & 30.67 \\
\hline
\end{tabular}

Após a coleta de dados no roteamento IP, os roteadores LSR2 e LSR3 foram reinicializados e passaram a utilizar um kernel com suporte ao protocolo MPLS. 
Para habilitar o suporte ao protocolo MPLS no Linux é necessário aplicar uma correção (patch) [43] ao seu código fonte. No período de execução deste trabalho, a versão mais recente dessa correção era a 1.946a, compatível com o kernel 2.6.9:

Essa correção irá adicionar novos parâmetros de configuração para a recompilação do kernel. As configurações necessárias para que o novo kernel suporte o protocolo MPLS são as seguintes:

- Em Code maturity level options, ativar opção Prompt for development and/or incomplete code/drivers. Essa opção precisa ser ativada porque a implementação do MPLS no Linux ainda está em desenvolvimento;

- Em Device Drivers $->$ Networking support $->$ Networking options, ativar o item Multiprotocol Label Switching;

- Em Device Drivers $->$ Networking support $->$ Networking options $->$ Network packet filtering (replaces ipchains) $->$ IP: Netfilter Configuration, ativar o item spec_nh target support. Este item é necessário para possibilitar a especificação de nexthops "especiais", do tipo MPLS Unicast, na fase de direcionamento de tráfego com o iptables.

Após a reinicialização dos computadores com o novo kernel, o suporte ao protocolo MPLS foi confirmado através dos seguintes comandos:

1sr2: \# cat /sys/mpls/version

1.946

lsr2: \# dmesg|grep MPLS

MPLS: version 1.946

O primeiro teste nesse cenário foi novamente sobre o roteamento IP. Os roteadores LSR2 e LSR3 foram configurados da mesma maneira que no roteamento IP com o kernel sem suporte ao MPLS. Esse teste foi realizado para avaliar o impacto da implementação, sem a utilização, por enquanto, do protocolo MPLS 
no software de rede do sistema operacional Linux. Podemos observar nos relatórios do tcpdump que mesmo com o protocolo MPLS ativado, todo o tráfego ocorre sobre datagramas IP, pois nenhum LSP foi configurado. Os resultados deste teste estão na tabela 5.3, e podemos observar que há pouca variação em relação à tabela 5.2 .

Tabela 5.3: Roteamento IP com dois nós intermediários e com MPLS

\begin{tabular}{|r|r|r|}
\hline \%CPU LSR2 & \%CPU LSR3 & Capacidade de roteamento (Mbps) \\
\hline 99 & 63 & 30,34 \\
\hline
\end{tabular}

O último teste realizado nessa rede com dois roteadores foi a utilização do roteamento IP sobre MPLS (figura 5.4). HOSTA e HOSTB continuam usando LSR2 e LSR3, respectivamente, como roteadores, pois a existência de um domínio MPLS é transparente para as estações. A diferença está na configuração dos roteadores.

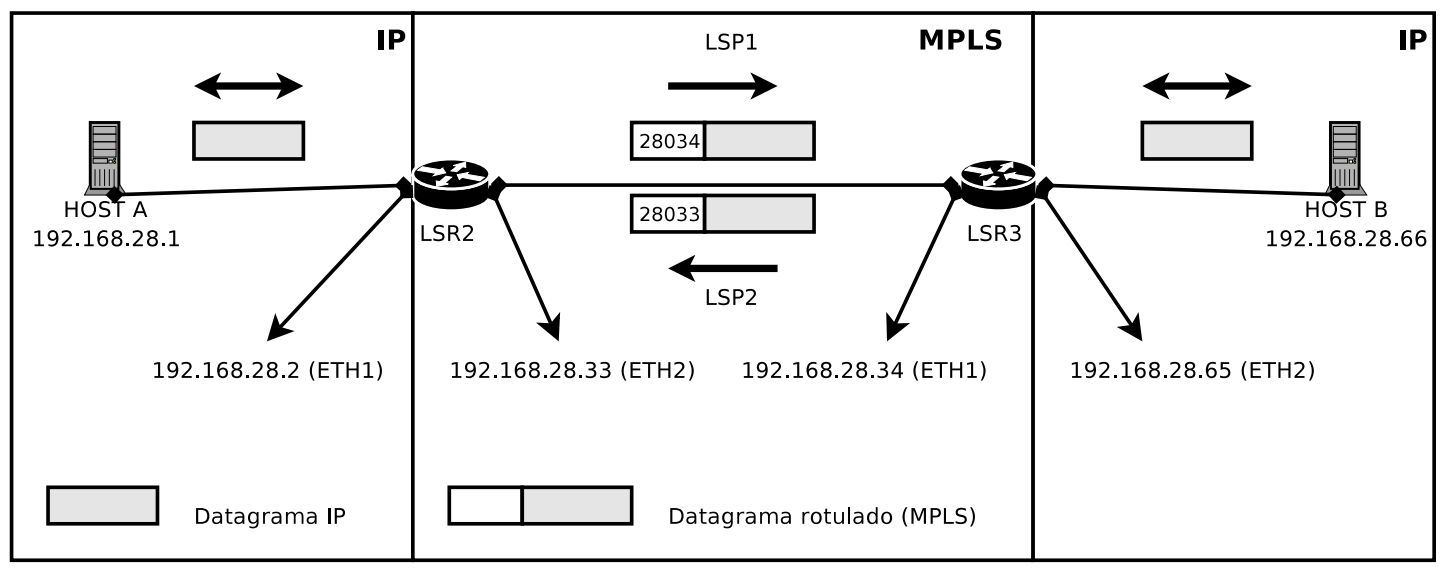

Figura 5.4: Dois roteadores MPLS

Inicialmente, podemos verificar pelos comandos a seguir que não há nenhuma configuração de rótulos de entrada ou de saída nos roteadores:

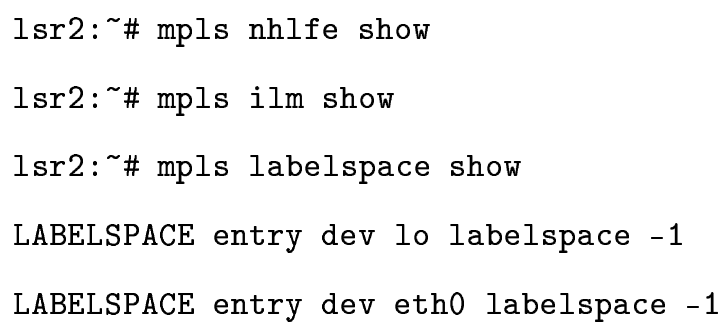




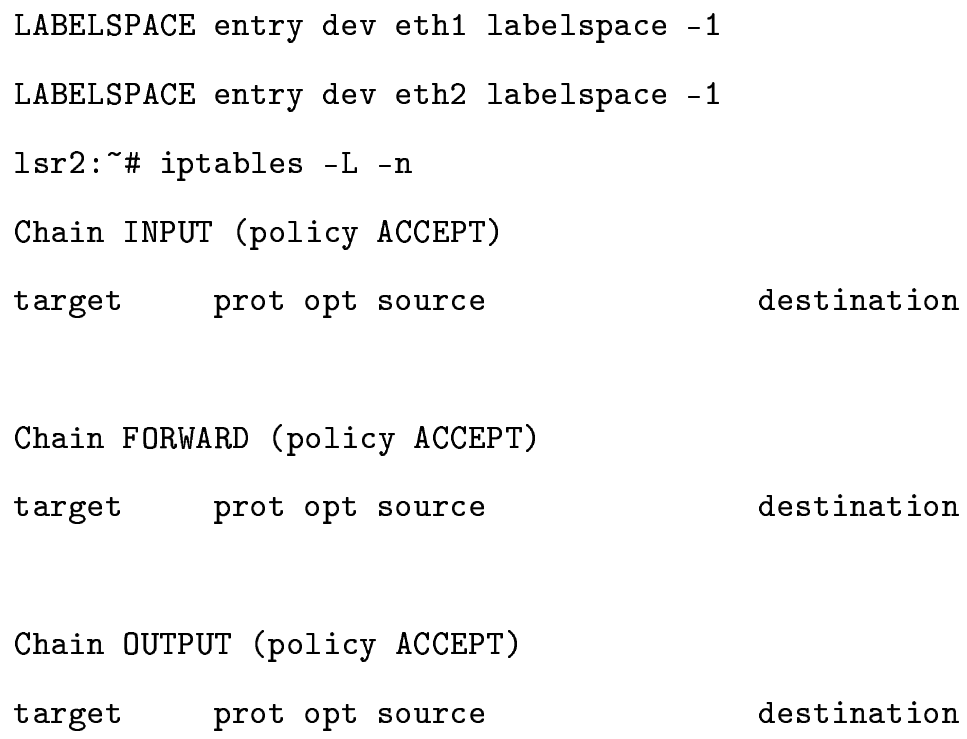

Uma vez ativado o suporte ao MPLS no kernel, é necessário criar os LSPs e direcionar o tráfego dos datagramas desejados para eles. Neste cenário serão criados dois LSPs: um no sentido de LSR2 para LSR3 (rótulo 28034) e outro no sentido de LSR3 para LSR2 (rótulo 28033).

Como LSR2 é um LER, ele precisa suportar roteamento IP para conseguir tratar datagramas IP que são recebidos ou encaminhados para fora do LSP. A criação da rota para a rede de HOSTB e a ativação do roteamento IP são feitas com os seguintes comandos:

1sr2: \# ip route add 192.168.28.64/27 nexthop via 192.168.28.34

1sr2: \# sysctl -w net.ipv4.ip_forward=1

A criação de um LSP na implementação Linux é feita através da ferramenta mpls [43]. A primeira configuração feita em LSR2 foi a criação de um rótulo com os seus respectivos nexthop e interface ethernet de saída:

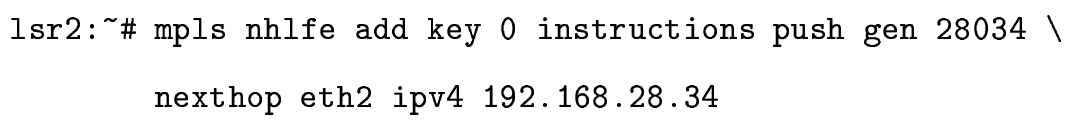

Key: 0x00000002

O comando acima cria uma nova entrada NHLFE (nhlfe add key 0 ) com instruções para adicionar um rótulo de valor 28034 (instructions push gen 28034) ao 
datagrama, e o encaminhamento para o próximo salto deve ser feito pela interface de rede eth2 para o endereço IP 192.168.28.34 (nexthop eth2 ipv4 192.168.28.34).

Outra função de LSR2 é retirar o cabeçalho MPLS de datagramas rotulados com o valor 28033 e encaminhá-los segundo as informações de rede (cabeçalho IP). O comando abaixo cria o mapeamento para esses rótulos de chegada:

lsr2: \# mpls ilm add label gen 28033

Se nenhum tipo de operação for especificada, a ferramenta mpls configura o mapeamento como uma operação de retirada (pop) do cabeçalho MPLS.

Para que ocorra a comunicação através do protocolo MPLS entre LSR2 e LSR3, ambos precisam configurar a interface de rede pertencente ao LSP (eth2) no mesmo labelspace (neste caso, zero). Isso é feito com o seguinte comando:

1sr2: \# mpls labelspace add dev eth2 labelspace 0

A depuração deve ser desabilitada, pois ela é um processo muito custoso para os LSRs e interferem nos resultados das medições de desempenho.

lsr2: \# echo $0>/$ sys/mpls/debug

O próximo passo é configurar LSR3. Essa configuração é muito semelhante à de LSR2, pois LSR3 também é um LER. As diferenças na configuração estão no valor do rótulo, posição das interfaces de rede e endereços IP utilizados:

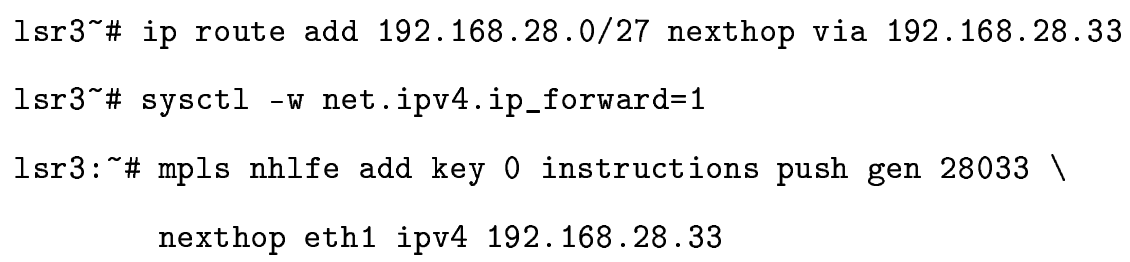

Key: 0x00000002

lsr $3^{\sim}$ \#pls ilm add label gen 28034

1sr $3^{\sim} \#$ mpls labelspace add dev eth1 labelspace 0

1sr3: \# echo $0>/$ sys/mpls/debug

Neste ponto, os rótulos de saída e de chegada já estão configurados, mas ainda não estão sendo utilizados: 
lsr2: \# mpls nhlfe show

NHLFE entry key 0x00000002 mtu 1496 propagate_ttl

push gen 28034 set eth2 ipv4 192.168.28.34 (0 bytes, 0 pkts, 0 dropped)

lsr2: \# mpls ilm show

ILM entry label gen 28033 labelspace 0 proto ipv4

pop peek (0 bytes, 0 pkts, 0 dropped)

1sr2: \# mpls labelspace show

LABELSPACE entry dev eth0 labelspace -1

LABELSPACE entry dev lo labelspace -1

LABELSPACE entry dev eth1 labelspace -1

LABELSPACE entry dev eth2 labelspace 0

1sr2: \# sysctl net.ipv4.ip_forward

net.ipv4.ip_forward $=1$

1sr2: \# netstat -rn

Kernel IP routing table

Destination Gateway

Genmask Flags MSS Window irtt Iface

$172.28 \cdot 0.0$

0.0 .0 .0

$255.255 .0 .0 \quad \mathrm{U}$

00

0 eth 0

192.168 .28 .0

0.0 .0 .0

$255.255 .255 .224 \mathrm{U}$

00

0 eth1

$192.168 .28 .32 \quad 0.0 .0 .0$

$255.255 .255 .224 \mathrm{U}$

00

0 eth2

192.168 .28 .64

192.168 .28 .34

255.255 .255 .224 UG

00

0 eth2

lsr3: \# mpls nhlfe show

NHLFE entry key 0x00000002 mtu 1496 propagate_ttl

push gen 28033 set eth1 ipv4 192.168.28.33 (0 bytes, 0 pkts, 0 dropped)

lsr3: \# mpls ilm show

ILM entry label gen 28034 labelspace 0 proto ipv4

pop peek (0 bytes, 0 pkts, 0 dropped)

lsr3: \# mpls labelspace show

LABELSPACE entry dev eth0 labelspace -1

LABELSPACE entry dev lo labelspace -1

LABELSPACE entry dev eth1 labelspace 0

LABELSPACE entry dev eth2 labelspace -1

1sr3: \# sysctl net.ipv4.ip_forward

net.ipv4.ip_forward $=1$

1sr3: \# netstat $-r n$ 


\begin{tabular}{|c|c|c|c|c|c|c|}
\hline Destination & Gateway & Genmask & Flags & MSS & Window & irtt Iface \\
\hline 172.28 .0 .0 & 0.0 .0 .0 & 255.255 .0 .0 & $\mathrm{U}$ & 0 & 0 & 0 etho \\
\hline 192.168 .28 .0 & 192.168 .28 .33 & 255.255 .255 .224 & UG & 0 & 0 & 0 eth1 \\
\hline 192.168 .28 .32 & 0.0 .0 .0 & 255.255 .255 .224 & $\mathrm{U}$ & 0 & 0 & 0 eth 1 \\
\hline 192.168 .28 .64 & 0.0 .0 .0 & 255.255 .255 .224 & $\mathrm{U}$ & 0 & 0 & 0 eth2 \\
\hline
\end{tabular}

Para que os datagramas sejam rotulados e passem a utilizar o LSP criado, é preciso que o tráfego desejado seja direcionado para esse LSP. Esse direcionamento é feito com a ferramenta iptables [44]. O iptables padrão das distribuições Linux não suportam os parâmetros de configuração de LSPs. Para que o iptables suporte esses parâmetros, existe uma correção disponível no site do projeto MPLS for Linux [43]. Os comandos de direcionamento dos tráfegos são o seguintes:

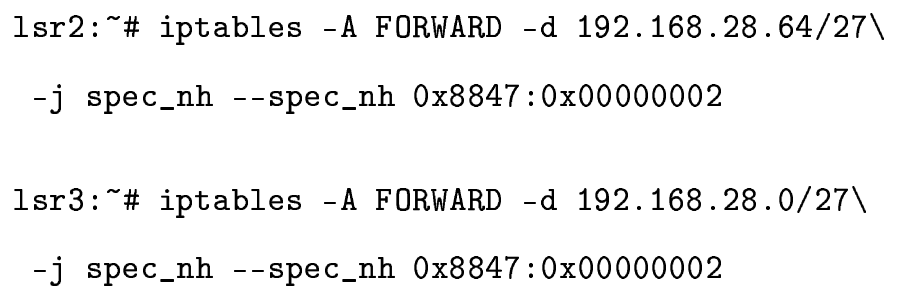

Neste cenário, LSR2 realiza duas operações:

- Direciona todo o tráfego cujo destino é a rede 192.168.28.64/27 para trafegar sobre o LSP;

- Retira cabeçalhos MPLS dos datagramas cujo rótulo possua o valor 28033.

LSR3 também realiza duas operações:

- Direciona todo o tráfego cujo destino é a rede 192.168.28.0/27 para trafegar sobre o LSP;

- Retira cabeçalhos MPLS dos datagramas cujo rótulo possua o valor 28034.

Novamente, para comprovar a utilização do protocolo MPLS nos roteadores, foram disparadas mensagens ICMP (ping) enquanto as interfaces de rede eram monitoradas com o tcpdump. 
O relatório gerado pelo tcpdump mostra que as interfaces eth1 de LSR2 e eth2 de LSR3 recebem datagramas IP contendo dois tipos de mensagens ICMP: echo request com origem em 192.168.28.1 e destino 192.168.28.66, e echo reply com origem 192.168.28.66 e destino 192.168.28.1. Essas interfaces não recebem datagramas rotulados porque elas estão fora do domínio MPLS, e se comunicam com as estações através do protocolo IP.

Já as interfaces eth2 de LSR2 e eth1 de LSR3 recebem somente datagramas rotulados, pois estão dentro do domínio MPLS. O rótulo de valor 28034 corresponde às mensagens ICMP echo request com origem em 192.168.28.1 e destino 192.168.28.66, enquanto que o rótulo de valor 28033 corresponde às mensagens echo reply com origem em 192.168.28.66 e destino 192.168.28.1.

No roteamento IP sobre MPLS, os LERs são submetidos a uma carga adicional, pois além de todo o processamento e análise das informações do protocolo IP, eles precisam associar cada tipo de datagrama à sua FEC correspondente e inserir o cabeçalho MPLS entre o cabeçalho IP e o cabeçalho Ethernet.

Como conseqüência, em uma rede com dois roteadores em funcionamento (figura 5.4), o desempenho é menor no roteamento IP sobre MPLS do que no roteamento IP direto sobre Ethernet. Os resultados obtidos neste teste de roteamento IP sobre MPLS estão na tabela 5.4.

Tabela 5.4: Capacidade de roteamento com dois nós intermediários

\begin{tabular}{|r|r|r|}
\hline \%CPU LSR2 & \%CPU LSR3 & Capacidade de roteamento (Mbps) \\
\hline 99 & 59 & 25,58 \\
\hline
\end{tabular}

LSR2 já estava trabalhando na sua capacidade máxima no roteamento IP. Portanto, ao receber a sobrecarga no roteamento IP sobre MPLS, sua capacidade de roteamento caiu, diminuindo a carga sobre LSR3. Isso pode ser comprovado pela queda na utilização de CPU em LSR3 (de $63 \%$ no roteamento IP para $59 \%$ no roteamento IP sobre MPLS).

Através da figura 5.5 podemos observar que a capacidade de roteamento é menor no roteamento IP sobre MPLS. Isso se deve ao fato desta topologia não 
possuir nós intermediários. Podemos observar também, que apenas a ativação do protocolo MPLS (sem direcionamento de tráfego para o LSP) no sistema operacional interfere muito pouco no desempenho dos roteadores.

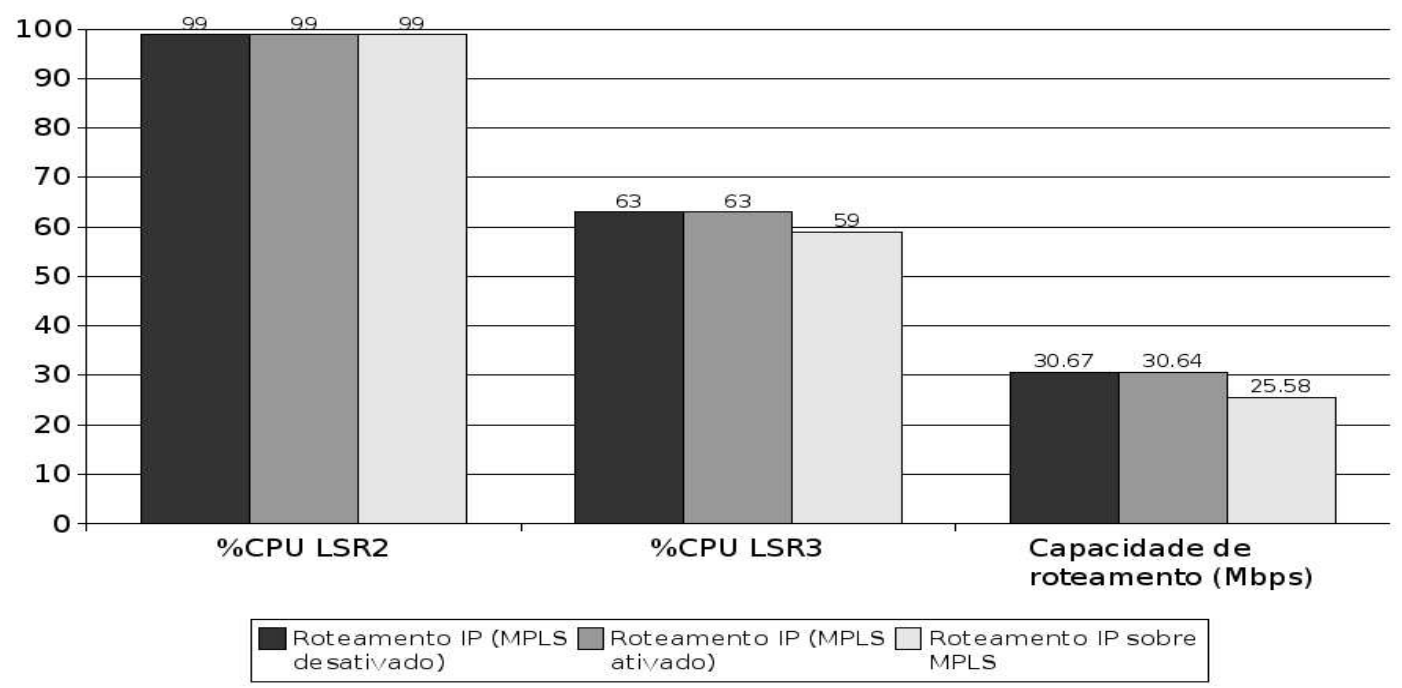

Figura 5.5: Desempenho dos roteadores

\subsection{Quantidade de nós intermediários}

Os nós beneficiados pela arquitetura IP sobre MPLS são os nós intermediários, pois eles não precisam analisar o cabeçalho IP, e trabalham apenas com os dados do cabeçalho MPLS. Para comprovar que o ganho de desempenho se dá nos nós intermediários, foram realizadas medições em redes com três roteadores, tanto em roteamento IP como em roteamento IP sobre MPLS.

A primeira medição foi da capacidade de roteamento IP, e os três roteadores foram configurados sem o suporte ao protocolo MPLS (figura 5.6).

Para esse cenário com três roteadores, as configurações de HOSTA e HOSTB sofreram pequenas modificações:

- HOSTB passa a pertencer à rede 192.168.28.96/27 e utilizar o endereço IP 192.168.28.98; 


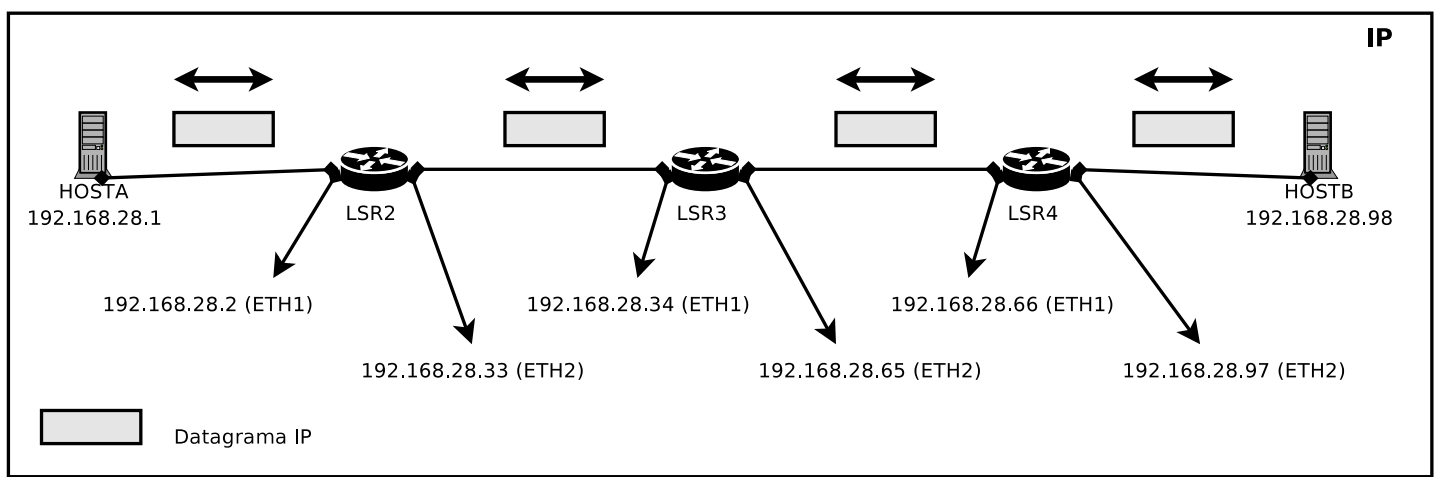

Figura 5.6: Roteamento IP com três roteadores

- Em HOSTB, o roteador para a rede 192.168.28.0/27 mudou de 192.168.28.65 para 192.168.28.97;

- Foi adicionada uma rota em HOSTA para a rede 192.168.28.96/27, através do roteador 192.168.28.2, e removida a rota para a rede 192.168.28.64/27.

hosta: \# netstat -rn

Kernel IP routing table

Destination Gateway

Genmask

Flags

MSS Window irtt Iface

192.168 .28 .0

$0.0 \cdot 0.0$

$255.255 .255 .224 \mathrm{U}$

00

0 eth1

192.168 .28 .96

192.168 .28 .2

255.255.255.224 UG

00

0 eth1

hostb: \# netstat - rn

Kernel IP routing table

Destination Gateway

Genmask Flags MSS Window irtt Iface

$192.168 .28 .96 \quad 0.0 .0 .0$

$255.255 .255 .224 \mathrm{U}$

00

0 eth1

192.168 .28 .0

192.168 .28 .97

$255.255 .255 .224 \mathrm{UG}$

00

0 eth1

O roteador LSR2 precisa de uma rota para a rede 192.168.28.96/27 através de 192.168.28.34 (LSR3-eth1), e o roteador LSR4 precisa de uma rota para a rede 192.168.28.0/27 através de 192.168.28.65 (LSR3-eth2).

1sr2: \# sysctl net.ipv4.ip_forward

net.ipv4.ip_forward $=1$

1sr2: \# netstat $-r n$

Kernel IP routing table

Destination Gateway

Genmask

Flags MSS Window irtt Iface 


\begin{tabular}{|c|c|c|c|c|c|c|c|}
\hline 172.28 .0 .0 & 0.0 .0 .0 & 255.255 .0 .0 & $\mathrm{U}$ & 0 & 0 & & eth0 \\
\hline 192.168 .28 .0 & 0.0 .0 .0 & 255.255 .255 .224 & $\mathrm{U}$ & 0 & 0 & 0 & eth1 \\
\hline 192.168 .28 .32 & 0.0 .0 .0 & 255.255 .255 .224 & $\mathrm{U}$ & 0 & 0 & 0 & eth2 \\
\hline 192.168 .28 .96 & 192.168 .28 .34 & 255.255 .255 .224 & UG & 0 & 0 & 0 & eth2 \\
\hline \multicolumn{8}{|c|}{ lsr4: \# sysctl net.ipv4.ip_forward } \\
\hline \multirow{2}{*}{\multicolumn{8}{|c|}{$\begin{array}{l}\text { lsr4: \# netstat }-\mathrm{rn} \\
\text { Kernel IP routing table }\end{array}$}} \\
\hline & & & & & & & \\
\hline Destination & Gateway & Genmask & Flags & MSS & Window & irtt & If ace \\
\hline 172.28 .0 .0 & 0.0 .0 .0 & 255.255 .0 .0 & $\mathrm{U}$ & 0 & 0 & 0 & eth0 \\
\hline 192.168 .28 .0 & 192.168 .28 .65 & 255.255 .255 .224 & UG & 0 & 0 & 0 & eth1 \\
\hline 192.168 .28 .64 & 0.0 .0 .0 & 255.255 .255 .224 & $\mathrm{U}$ & 0 & 0 & 0 & eth1 \\
\hline 192.168 .28 .96 & 0.0 .0 .0 & 255.255 .255 .224 & $\mathrm{U}$ & 0 & 0 & 0 & eth2 \\
\hline
\end{tabular}

O roteador LSR3 precisa de rotas para as duas redes (192.168.28.0/27 e 192.168.28.96/27), pois não está diretamente conectado a nenhuma delas:

\begin{tabular}{|c|c|c|c|c|c|c|c|}
\hline \multicolumn{8}{|c|}{ 1sr3: \# netstat -rn } \\
\hline \multicolumn{8}{|c|}{ Kernel IP routing table } \\
\hline Destination & Gateway & Genmask & Flags & MSS & Window & irtt & If ace \\
\hline 172.28 .0 .0 & 0.0 .0 .0 & 255.255 .0 .0 & $\mathrm{U}$ & 0 & 0 & & eth0 \\
\hline 192.168 .28 .0 & 192.168 .28 .33 & 255.255 .255 .224 & UG & 0 & 0 & & eth1 \\
\hline 192.168 .28 .32 & 0.0 .0 .0 & 255.255 .255 .224 & $\mathrm{U}$ & 0 & 0 & & eth1 \\
\hline 192.168 .28 .64 & 0.0 .0 .0 & 255.255 .255 .224 & $\mathrm{U}$ & 0 & 0 & & eth2 \\
\hline 192.168 .28 .96 & 192.168 .28 .66 & 255.255 .255 .224 & UG & 0 & 0 & & eth2 \\
\hline
\end{tabular}

Com as rotas necessárias configuradas, a rede foi testada com a ferramenta netperf, e os resultados estão na tabela 5.5.

Tabela 5.5: Roteamento IP com três LSRs sem MPLS

\begin{tabular}{|r|r|r|r|}
\hline \%CPU LSR2 & \%CPU LSR3 & \%CPU LSR4 & Capacidade de roteamento (Mbps) \\
\hline 99 & 62 & 40 & 32,15 \\
\hline
\end{tabular}


Como podemos observar na tabela 5.5, LSR2 está em $99 \%$ de utilização de Central Processing Unit (CPU), e a capacidade de roteamento desta rede está próxima à capacidade de roteamento de LSR2 (figura 5.2).

O próximo teste foi feito em arquiteturas IP sobre MPLS. Após as medições do roteamento IP, os roteadores foram configurados para suportar o protocolo MPLS (figura 5.7).

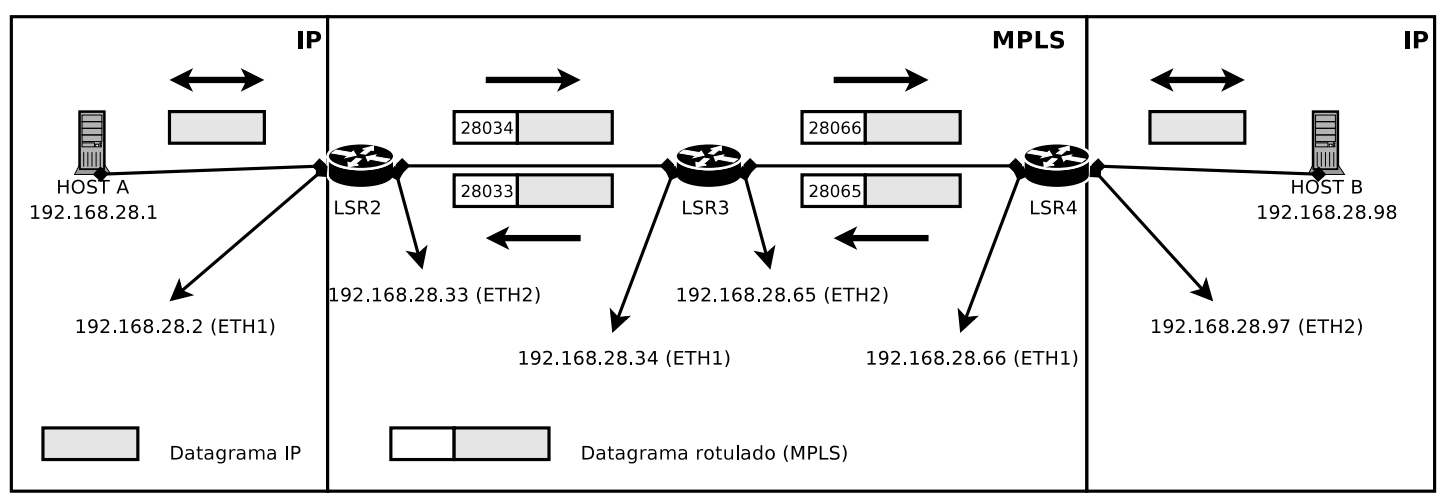

Figura 5.7: Três LSRs

HOSTA e HOSTB não sofreram alterações na configuração. Os LERs possuem configurações semelhantes entre si. LSR2 precisa rotular datagramas com destino à rede 192.168.28.96/27 com o valor 28034, e retirar cabeçalhos MPLS com o valor de rótulo 28033 .






\begin{tabular}{|c|c|c|c|c|c|c|c|}
\hline Destination & Gateway & Genmask & Flags & MSS & Window & irtt & Iface \\
\hline 172.28 .0 .0 & 0.0 .0 .0 & 255.255 .0 .0 & $\mathrm{U}$ & 0 & 0 & 0 & eth0 \\
\hline 192.168 .28 .0 & 0.0 .0 .0 & 255.255 .255 .224 & $\mathrm{U}$ & 0 & 0 & 0 & eth1 \\
\hline 192.168 .28 .32 & 0.0 .0 .0 & 255.255 .255 .224 & $\mathrm{U}$ & & 0 & & eth2 \\
\hline 192.168 .28 .96 & 192.168 .28 .34 & 255.255 .255 .224 & UG & 0 & 0 & & eth2 \\
\hline
\end{tabular}

LSR4 precisa rotular datagramas com destino à rede 192.168.28.0/27 com o valor 28065, e retirar cabeçalhos MPLS com o valor de rótulo 28066.

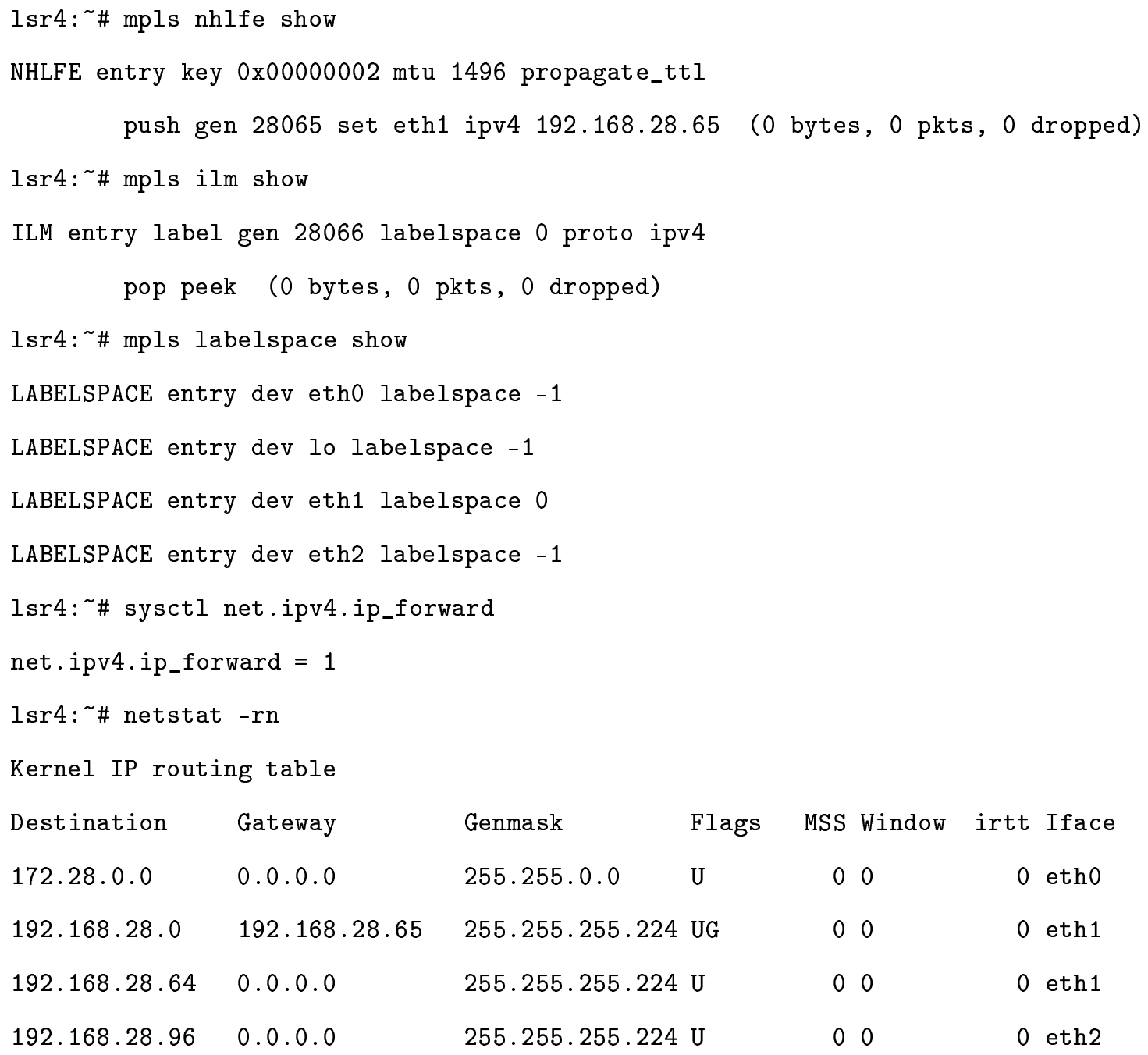

LSR3 está no meio do LSP, e precisa tratar datagramas rotulados em ambas as interfaces (eth1 e eth2). As operações que devem ocorrer em LSR3 são: 
- Receber datagramas com valor de rótulo 28034, trocar pelo valor 28066 e encaminhá-los para LSR4 (192.168.28.66) através da interface eth2;

- Receber datagramas com valor de rótulo 28065, trocar pelo valor 28033 e encaminhá-los para LSR2 (192.168.28.33) através da interface eth1.

Como LSR3 irá trabalhar apenas com datagramas rotulados, o suporte ao roteamento IP pode ser desabilitado.

lsr3: \# sysctl -w net.ipv4.ip_forward=0

Os comandos seguintes configuram os dois rótulos de saída de LSR3:

1sr3: \# mpls nhlfe add key 0 instructions push gen 28033\

nexthop eth1 ipv4 192.168.28.33

Key: 0x00000002

lsr3: \# mpls nhlfe add key 0 instructions push gen 28066\

nexthop eth2 ipv4 192.168.28.66

Key: 0x00000003

O resultado desses dois comandos é a criação de duas entradas (NHLFE) na tabela de encaminhamentos de LSR3:

O primeiro comando cria uma nova entrada NHLFE com instruções para adicionar um rótulo de valor 28033 ao datagrama, e o encaminhamento para o próximo salto deve ser feito pela primeira interface de rede (eth1) para o endereço IP 192.168.28.33.

O segundo comando também adiciona mais uma entrada NHLFE, só que com valores diferentes de rótulo (28066), interface de rede Ethernet (eth2) e próximo salto (192.168.28.66).

As entradas na tabela de NHLFEs já foram criadas, mas ainda não há o relacionamento delas com os rótulos de chegada. Os comandos abaixo mapeiam os rótulos de entrada aos rótulos de saída:

1sr3: \# mpls ilm add label gen 28065 instructions pop forward 0x00000002

1sr3: \# mpls ilm add label gen 28034 instructions pop forward 0x00000003 
O primeiro comando instrui LSR3 para trocar rótulos de valor 28065 pelo valor do rótulo correspondente ao registro NHLFE de chave 0x00000002 (28033), e o segundo comando instrui LSR3 para trocar rótulos de valor 28034 pelo valor do rótulo correspondente ao registro NHLFE de chave 0x00000003 (28066).

As duas interfaces de LSR3 (eth1 e eth2) fazem parte do LSP, portanto, devem receber o mesmo valor de labelspace.

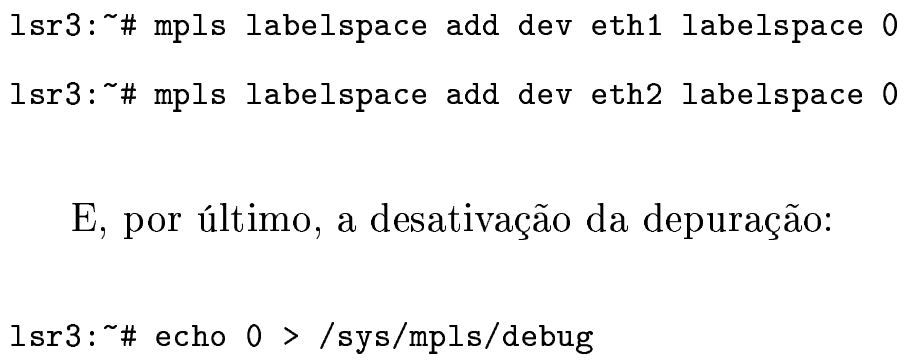

E, por último, a desativação da depuração:

1sr3: \# echo $0>/$ sys/mpls/debug

Após a execução dos comandos anteriores em LSR3, sua configuração é a seguinte:

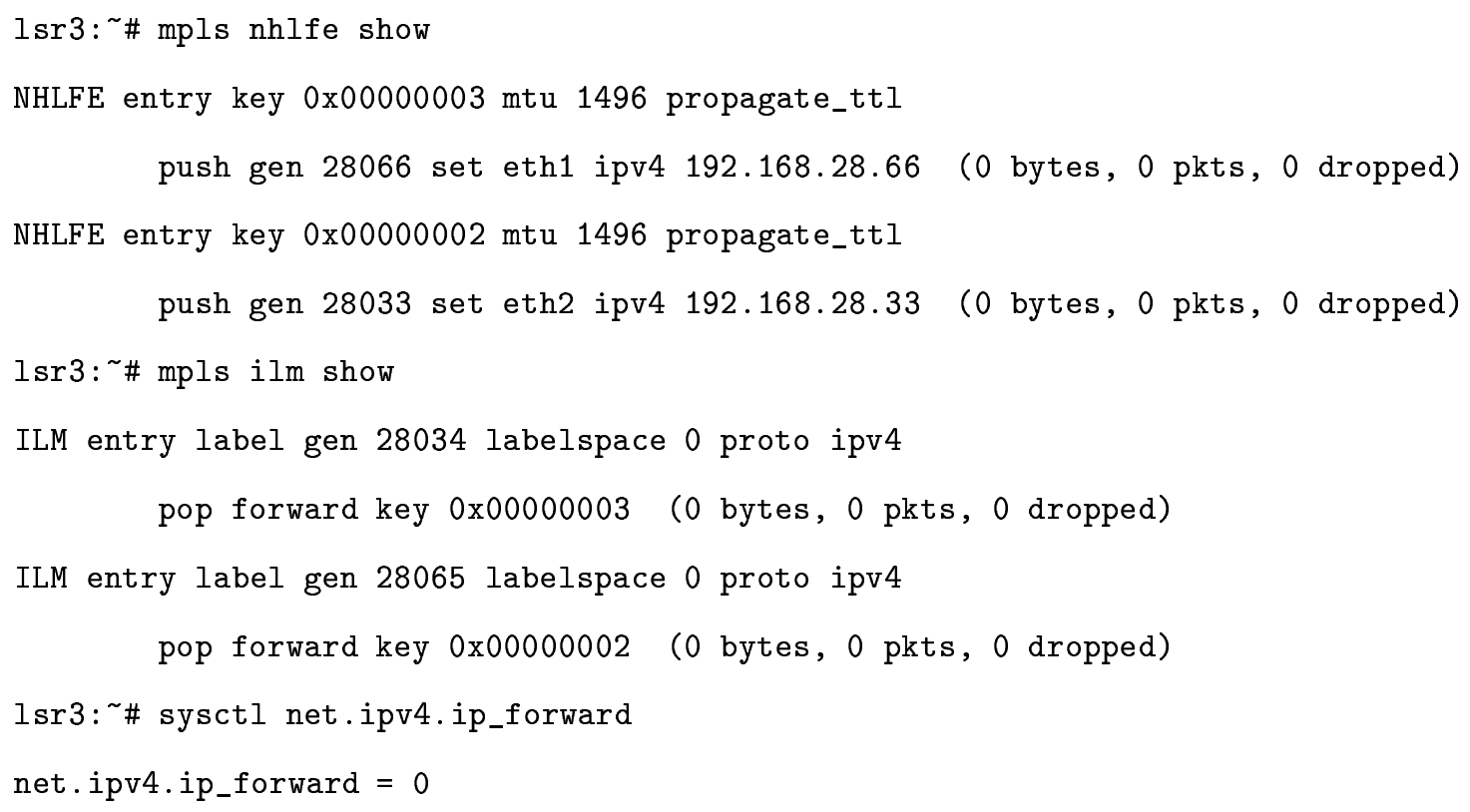

Com a rede ilustrada pela figura 5.7 configurada, foram disparadas mensagens ICMP com a ferramenta ping entre as duas estações, e as interfaces de rede dos LSRs foram monitoradas com a ferramenta tcpdump, a fim de se confirmar a utilização do protocolo MPLS no roteamento. 


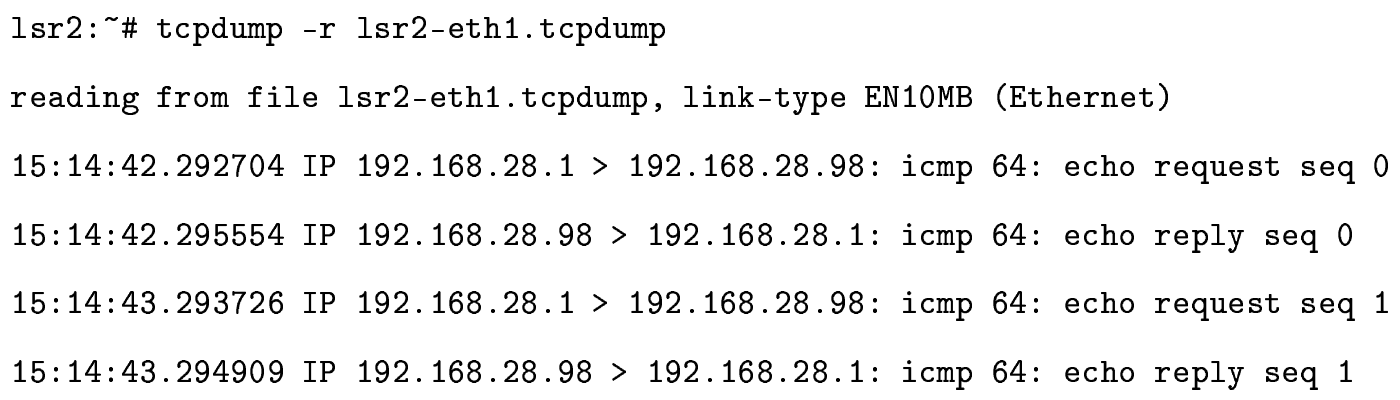

Neste cenário, temos os seguintes comportamentos em LSR2:

- A interface eth1 não recebe datagramas rotulados porque está fora do domínio MPLS;

- A interface eth2 recebe apenas datagramas rotulados porque está dentro do domínio MPLS;

- O rótulo de valor 28034 corresponde às mensagens ICMP echo request com origem em 192.168.28.1 e destino 192.168.28.98;

- O rótulo de valor 28033 corresponde às mensagens echo reply com origem em 192.168.28.98 e destino 192.168.28.1.

Em LSR4 a situação é semelhante à de LSR2:

- A interface eth2 não recebe datagramas rotulados porque está fora do domínio MPLS;

- A interface eth1 recebe apenas datagramas rotulados porque está dentro do domínio MPLS; 
- O rótulo de valor 28066 corresponde às mensagens ICMP echo request com origem em 192.168.28.1 e destino 192.168.28.98;

- O rótulo de valor 28065 corresponde às mensagens echo reply com origem em 192.168.28.98 e destino 192.168.28.1.

Já em LSR3, a situação é um pouco diferente, devido ao fato deste LSR estar no meio do LSP. As duas interfaces de rede (eth1 e eth2) não trabalham com o cabeçalho IP, apenas com o cabeçalho MPLS. Para garantir que não ocorra roteamento IP em LSR3, ele foi desabilitado no sistema operacional através da ferramenta sysctl.

$\mathrm{Na}$ interface eth1, os datagramas rotulados com 28034 correspondem ao echo request de 192.168.28.1 para 192.168.28.98 que estão sendo recebidos de LSR2, e os datagramas rotulados com 28033 correspondem ao echo reply de 192.168.28.98 para 192.168.28.1 que estão sendo enviados para LSR2. Em eth2, o valor de rótulo 28066 corresponde ao echo request de 192.168.28.1 para 192.168.28.98 que estão sendo enviados para LSR4, e 28065 corresponde ao echo reply de 192.168.28.98 para 192.168.28.1 que estão sendo recebidos de LSR4.

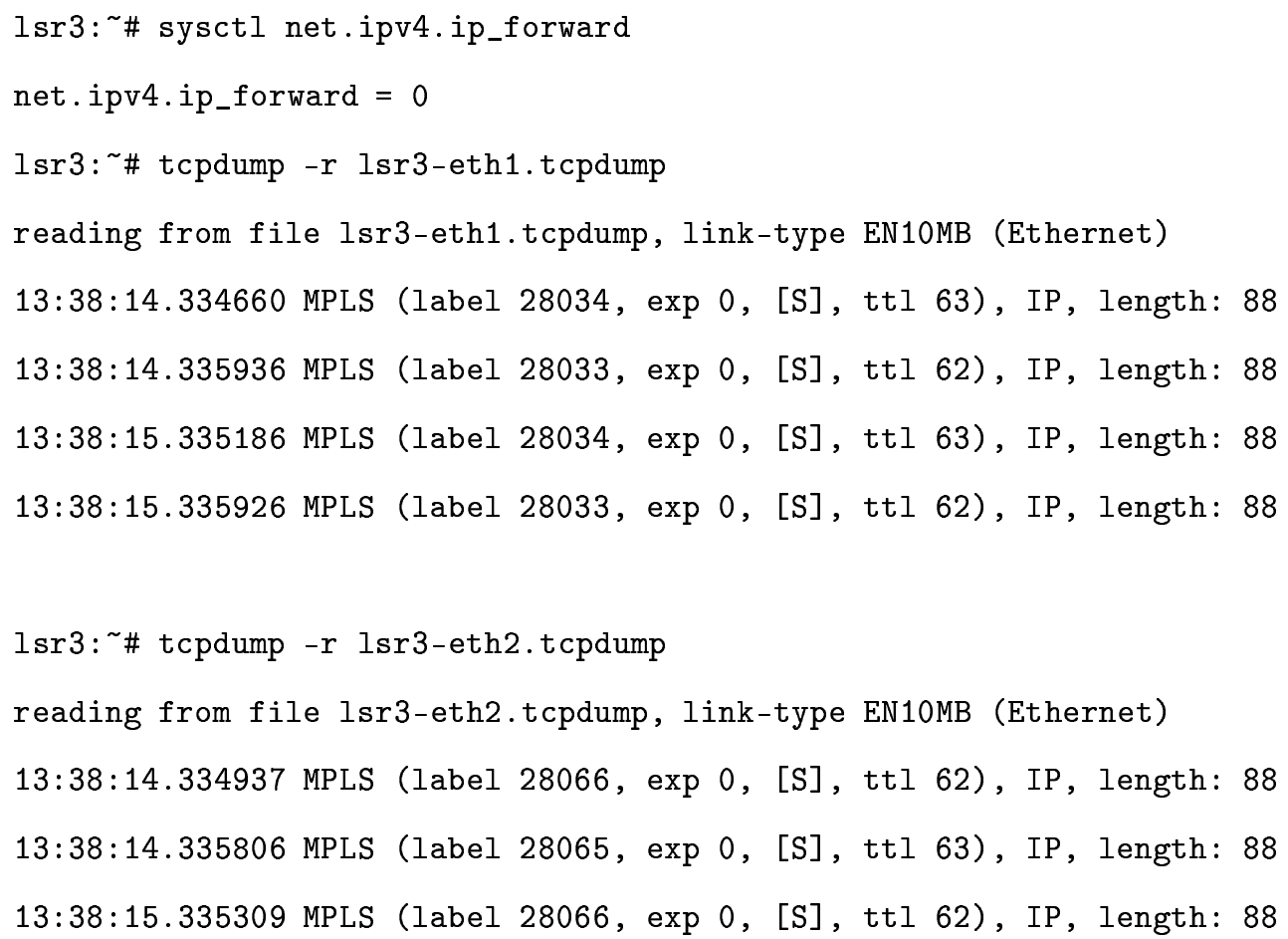


13:38:15.335775 MPLS (label 28065, exp 0, [S], ttl 63), IP, length: 88

Os resultados dos testes de roteamento IP sobre MPLS realizados na rede ilustrada pela figura 5.7 estão na tabela 5.6.

Tabela 5.6: Roteamento IP sobre MPLS com três LSRs

\begin{tabular}{|r|r|r|r|}
\hline \%CPU LSR2 & \%CPU LSR3 & \%CPU LSR4 & Capacidade de roteamento (Mbps) \\
\hline 99 & 40 & 61 & 25,62 \\
\hline
\end{tabular}

A figura 5.8 ilustra graficamente os resultados dos testes nas duas arquiteturas de roteamento (IP e IP sobre MPLS).

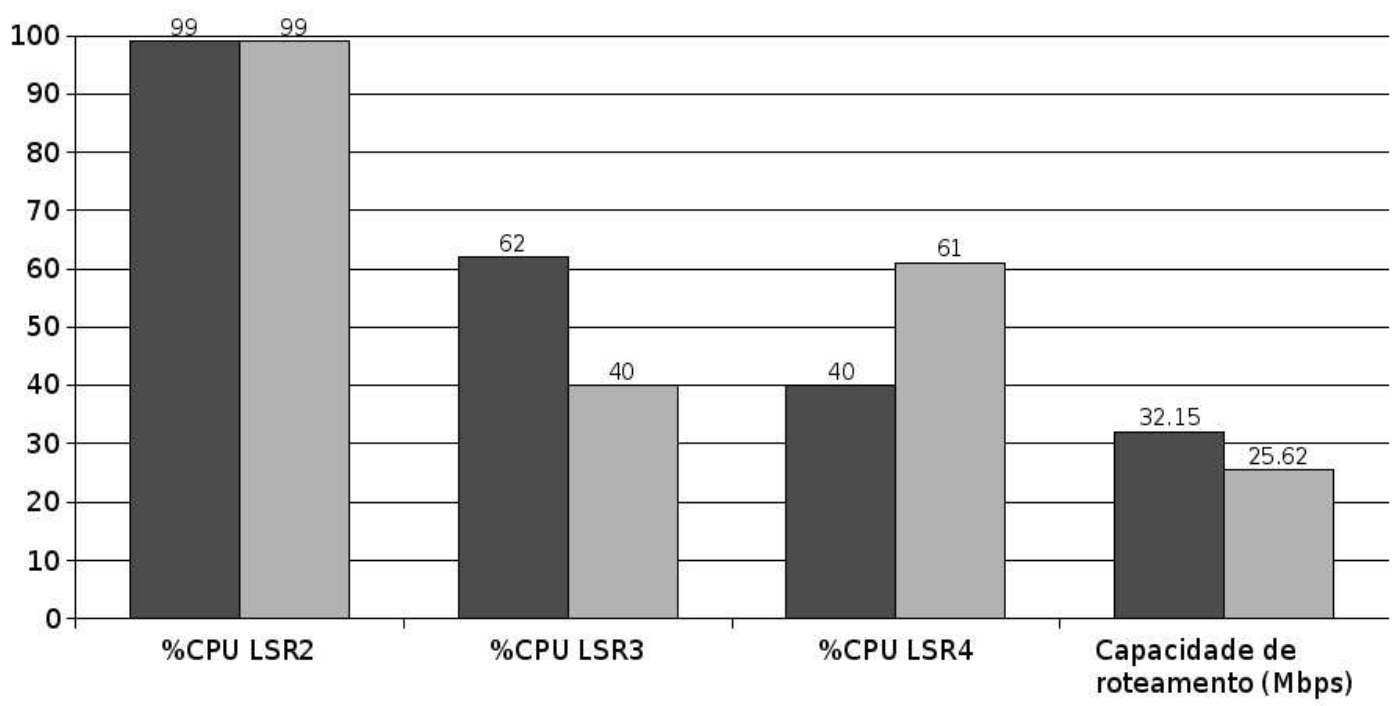

Roteamento IP $\quad \square$ Roteamento IP sobre

MPLS

Figura 5.8: Roteamento com roteadores em posições inadequadas

O primeiro fato interessante que podemos observar na figura 5.8 é que a maior capacidade de roteamento alcançada nesta rede é de $32,15 \mathrm{Mbps}$, mesmo com todos os nós equipados com placas de 100Mbps. Esse comportamento já era esperado, segundo os dados da figura 5.2, pois o roteador LSR2 tem baixo poder de processamento e conseguiu transmitir a apenas $31,74 \mathrm{Mbps}$. Os valores "\% CPU LSR2" da figura 5.8 (acima de 90\%) comprovam que apesar da interface de rede suportar um tráfego maior, o poder de processamento de LSR2 está limitando o tráfego da rede como um todo. 
A figura 5.8 mostra ainda que houve uma perda de desempenho no roteamento IP sobre MPLS (de 32,15Mbps para 25,62Mbps) mesmo havendo três nós na rede. Essa perda se deve à ativação do protocolo MPLS, pois o nó de entrada (LSR2, que já era o fator limitante do roteamento IP) recebeu uma carga adicional de processamento (adicionar/retirar o cabeçalho MPLS do datagrama). LSR4 também recebe uma carga adicional, já que é o egress LER desse LSP e precisa adicionar/retirar o cabeçalho MPLS dos datagramas.

Isso pode ser confirmado pelos valores da utilização de CPU dos roteadores (figura 5.8): em LSR2 manteve-se em 99\% (ele já estava no seu limite máximo de utilização) e em LSR4 subiu para 61\%. Em LSR3, a utilização de CPU caiu para 40\%, pois ele não mais analisa cabeçalhos IP, apenas cabeçalhos MPLS. Além disso, a capacidade de roteamento de LSR2 diminuiu, deixando LSR3 mais ocioso. Portanto, o fator limitante do tráfego nesta rede é a capacidade de processamento de LSR2.

Esse cenário não é favorável à implementação do roteamento IP sobre MPLS, pois o nó "gargalo" (LSR2) não está sendo beneficiado pela diminuição de processamento oferecido pelo protocolo MPLS.

Para comprovar (e mensurar) o ganho de desempenho através da utilização do protocolo MPLS, LSR2 e LSR3 foram reconfigurados e tiveram suas posições invertidas. Com essa inversão, o nó mais fraco ficou no meio do LSP e não irá mais analisar datagramas de rede.

De acordo com os resultados ilustrados pela figura 5.9, nesta situação houve um ganho de desempenho na rede com a utilização de roteamento IP sobre MPLS. Esta distribuição dos equipamentos é mais adequada, pois o nó mais fraco (LSR2) se beneficia do fato de não precisar analisar cabeçalhos IP.

O mesmo teste foi realizado em uma rede com 4 nós (figura 5.10). No roteamento IP, os relatórios emitidos pelo tcpdump mostraram que em todos os roteadores houve apenas tráfego IP.

No roteamento IP sobre MPLS, podemos identificar a presença de cabeçalhos 


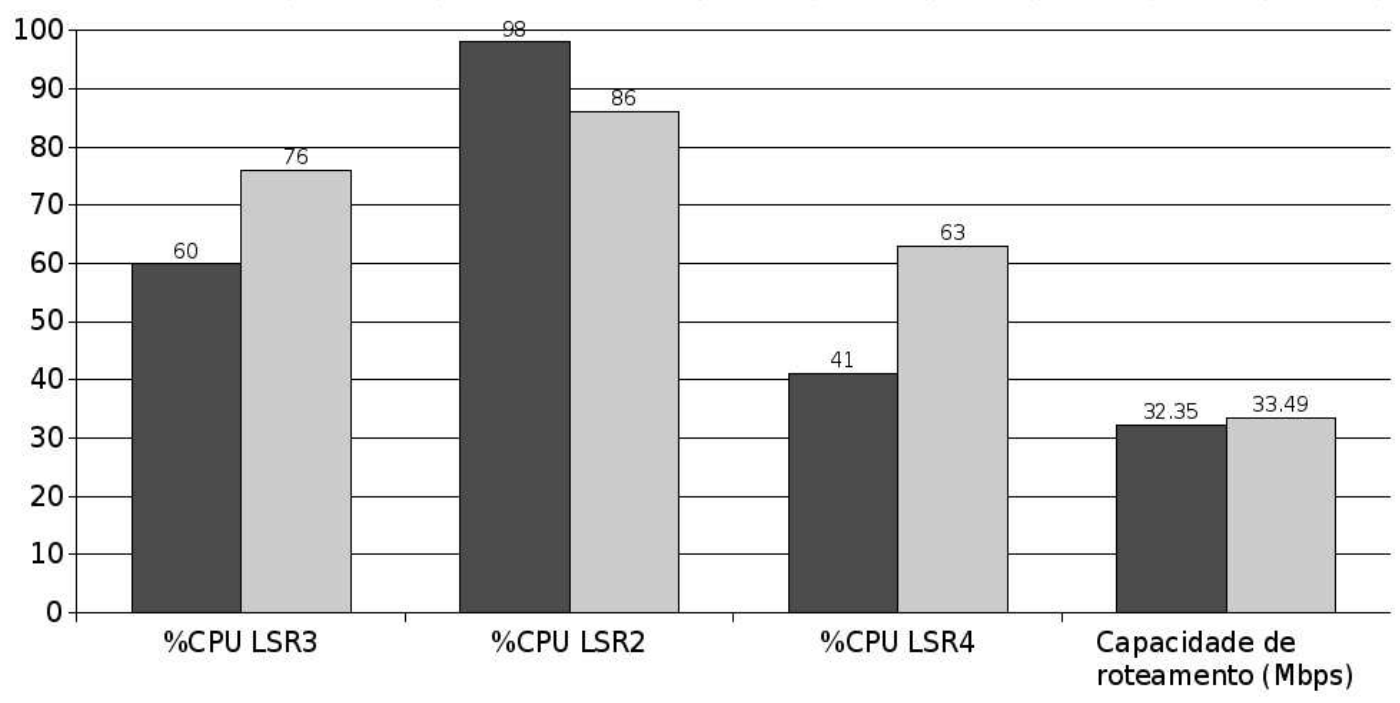

Roteamento IP $\square$ Roteamento IP sobre

MPLS

Figura 5.9: Roteamento em posições adequadas

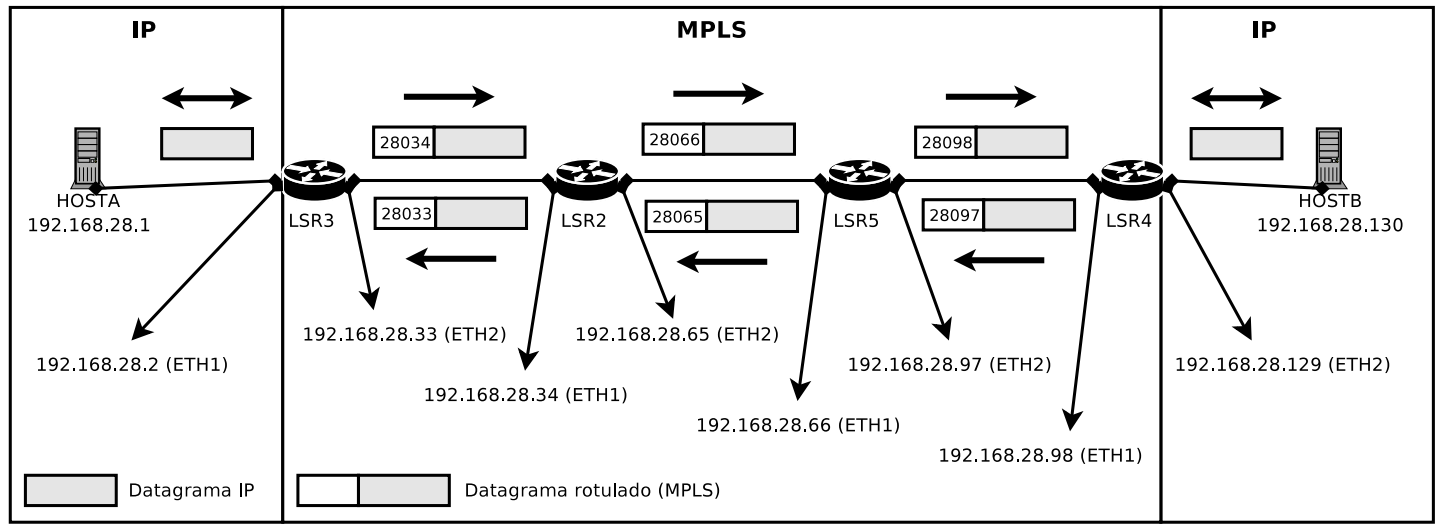

Figura 5.10: Quatro nós MPLS

MPLS "encapsulando" os datagramas IP.

Em LSR3, os relatórios do tcpdump mostraram que houve tráfego de datagramas IP na interface eth1 e de datagramas rotulados (28034 e 28033) na interface eth2.

Em LSR2 e LSR5 o roteamento IP foi desativado e trafegaram apenas datagramas rotulados (rótulos 28034, 28033, 28066 e 28065 em LSR2 e rótulos 28066, 28065, 28098 e 28097 em LSR5).

E finalmente, em LSR4, verificamos que houve tráfego de datagramas rotulados (28098 e 28097) em eth1 e datagramas IP em eth2. O ganho de desempenho 
obtido nesta rede (figura 5.11) é semelhante aos da rede com 3 nós (figura 5.9.

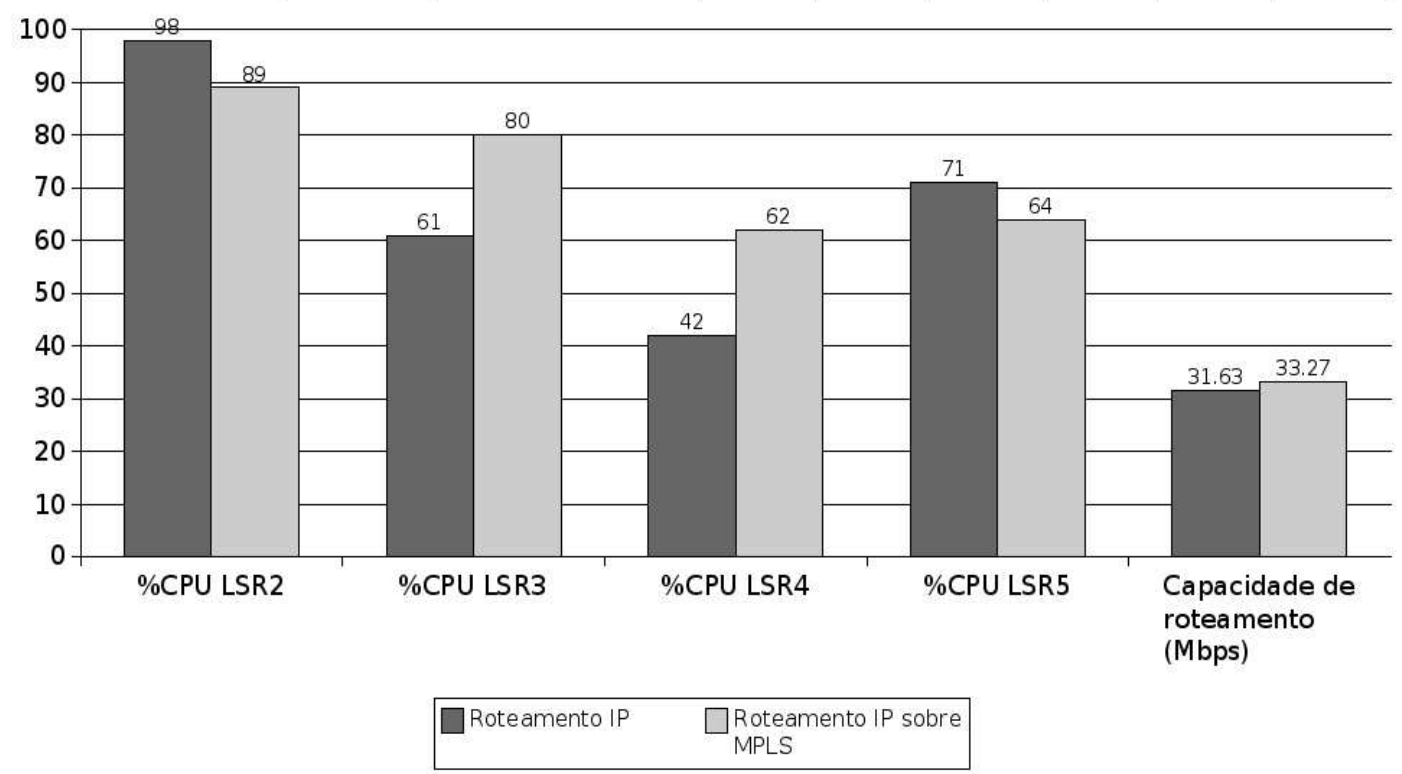

Figura 5.11: Quatro LSRs

Neste cenário o ganho de desempenho é um pouco maior que o roteamento IP, pois dois nós são beneficiados pela utilização do protocolo MPLS: LSR2 e LSR5. Além de aumentar a capacidade de tráfego nessa rede, o processamento nesses dois nós diminuiu.

Por outro lado, LSR3 e LSR4 (LERs) tiveram, novamente, um aumento na utilização de CPU devido à sobrecarga do protocolo MPLS nos roteadores de borda.

\section{$5.4 \quad$ Engenharia de tráfego}

O principal objetivo dos testes desta seção é avaliar o ganho de desempenho que a arquitetura IP sobre MPLS proporciona através da diferenciação de tráfego em uma granularidade mais fina que a do roteamento IP convencional.

Todos os testes desta seção foram feitos em uma rede do tipo "peixe", ilustrada pela figura 5.12. HOSTA continua pertencendo à rede 192.168.28.0/27, com o endereço IP 192.168.28.1. HOSTB passa a pertencer à rede 192.168.31.0/27 e 
recebe o endereço IP 192.168.31.1. HOSTA foi configurado para utilizar LSR1 (192.168.28.2) como roteador para a rede de HOSTB, e este foi configurado para utilizar LSR5 (192.168.31.2) como roteador para a rede de HOSTA.
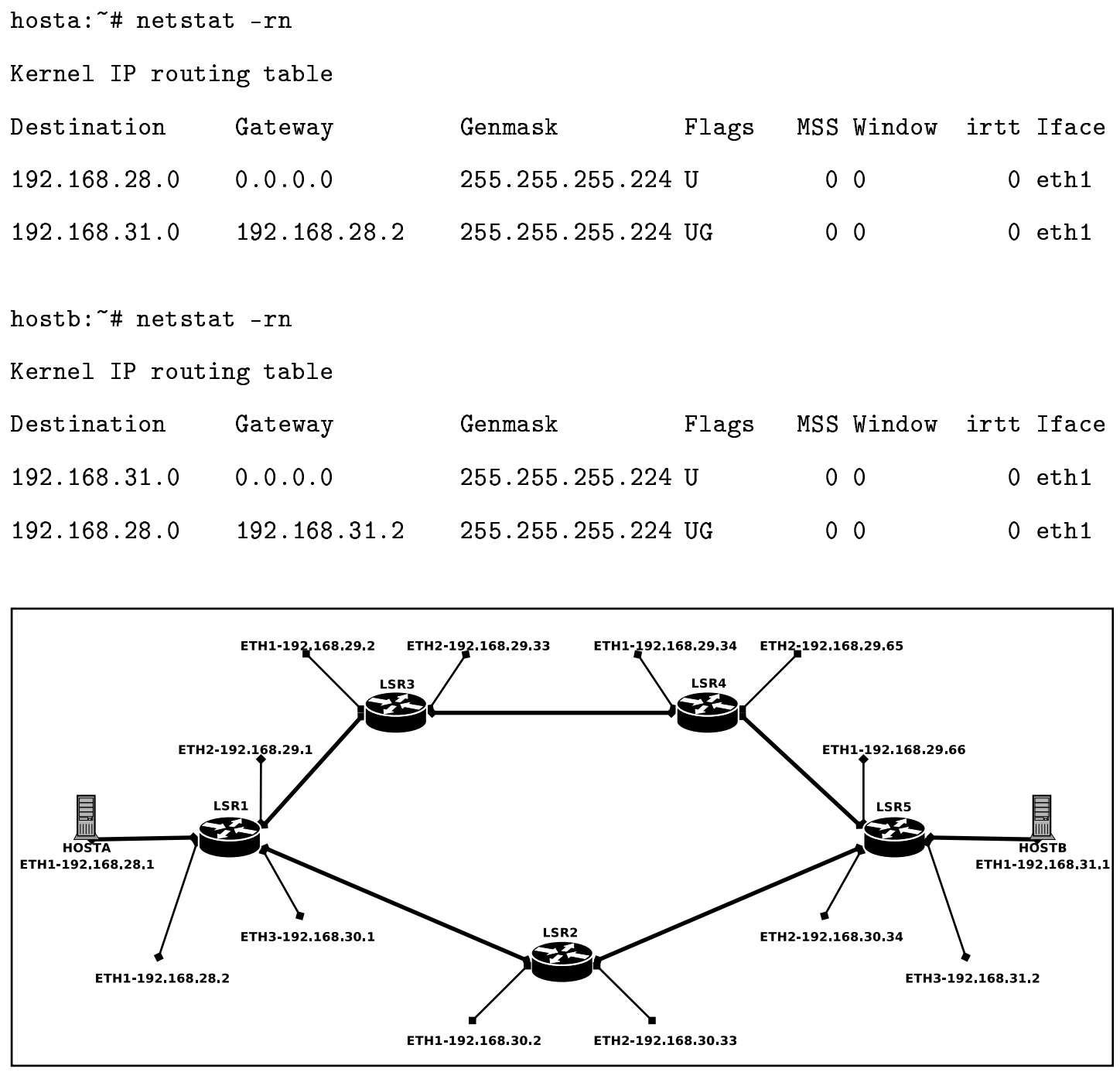

Figura 5.12: Rede "peixe"

Neste cenário foram utilizados dois computadores que trocam informações entre si através de duas aplicações TCP distintas.

A primeira avaliação foi do roteamento IP, onde ambas as conexões seguem pelo mesmo caminho (figura 5.13 ou figura 5.14), pois a decisão de roteamento é feita com base no endereço IP destino.

Para a utilização da rota A (LSR1-LSR2-LSR5), LSR1 foi configurado para utilizar LSR2 (192.168.30.2) como roteador para a rede de HOSTB: 


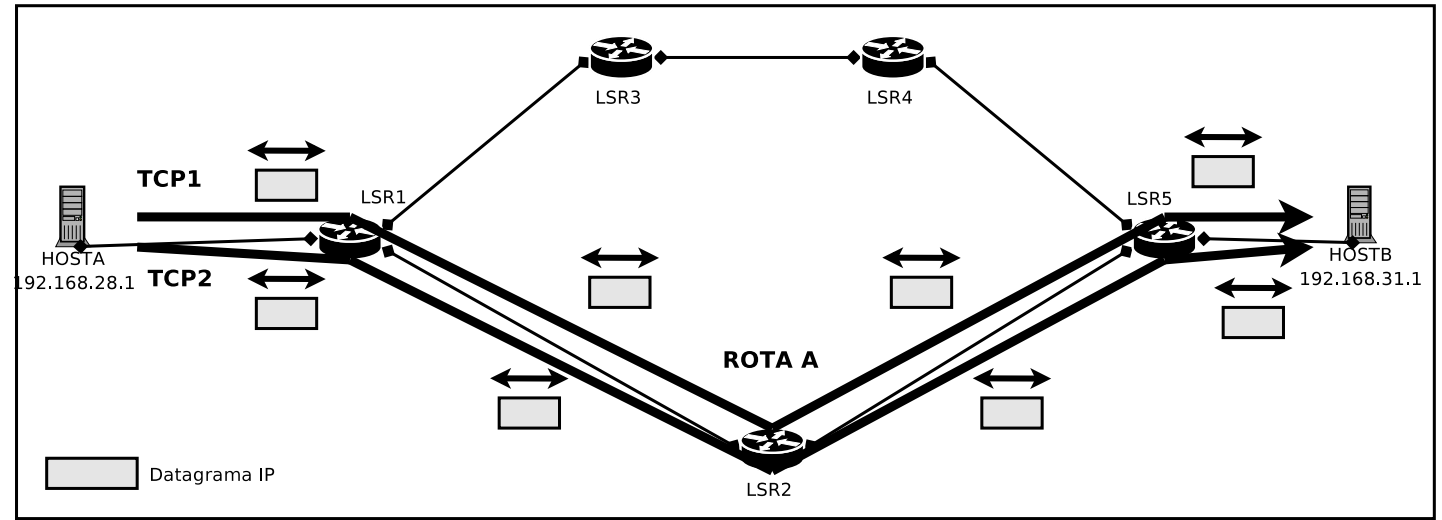

Figura 5.13: Roteamento IP: congestionamento na rota A

Isr1: \# netstat -rn

Kernel IP routing table

Destination Gateway

Genmask

Flags MSS Window irtt Iface

192.168 .28 .0

0.0 .0 .0

$255.255 .255 .224 \mathrm{U}$

00

0 eth1

192.168 .29 .0

$0.0 \cdot 0.0$

$255.255 .255 .224 \mathrm{U}$

00

0 eth2

192.168 .30 .0

$0.0 \cdot 0.0$

$255.255 .255 .224 \mathrm{U}$

00

0 eth3

192.168 .31 .0

192.168 .30 .2

$255.255 .255 .224 \mathrm{UG}$

00

0 eth3

Em LSR2, é necessário configurar rotas para alcançar HOSTA e HOSTB:

1sr2: \# netstat -rn

Kernel IP routing table

\begin{tabular}{|c|c|c|c|c|c|c|}
\hline Destination & Gateway & Genmask & Flags & MSS & Window & irtt \\
\hline 192.168 .28 .0 & 192.168 .30 .1 & 255.255 .255 .224 & UG & 0 & 0 & 0 \\
\hline 192.168 .30 .0 & 0.0 .0 .0 & 255.255 .255 .224 & & 0 & 0 & 0 \\
\hline 192.168 .30 .32 & 0.0 .0 .0 & 255.255 .255 .224 & $\mathrm{U}$ & 0 & 0 & 0 \\
\hline 192.168 .31 .0 & 192.168 .30 .34 & 255.255 .255 .224 & UG & 0 & 0 & 0 \\
\hline
\end{tabular}

E LSR5 precisa de uma rota para a rede de HOSTA:

1sr5: \# netstat $-r n$

Kernel IP routing table

Destination Gateway

Genmask

Flags

MSS Window irtt Iface

192.168 .28 .0

192.168 .30 .33

255.255.255.224 UG

00

0 eth2

192.168 .30 .32

0.0 .0 .0

$255.255 .255 .224 \mathrm{U}$

00

0 eth2

192.168 .29 .64

0.0 .0 .0

$255.255 .255 .224 \mathrm{U}$

00

0 eth 1

192.168 .31 .0

0.0 .0 .0

$255.255 .255 .224 \mathrm{U}$

00

0 eth3 
LSR3 e LSR4 não fazem parte da rota A.

Para se certificar de que os datagramas estão trafegando pela rota $\mathrm{A}$, todos os roteadores foram monitorados com tcpdump durante o envio de mensagens ICMP de HOSTA para HOSTB, através da ferramenta ping.

Os relatórios gerados pelo tcpdump mostram que as interfaces eth2 de LSR1 e eth1 de LSR5 não receberam nenhum tráfego, confirmando a utilização apenas da rota A. Além dos roteadores da rota A, LSR3 e LSR4 também foram monitorados com tcpdump, e nenhum tráfego foi registrado.

Com a rede configurada, foram executados dois processos netperf de HOSTA em direção a HOSTB. Cada processo utilizou uma porta TCP, onde TCP1 utilizou a porta 7000 e TCP2, a porta 7001. Como está sendo utilizado o roteamento IP, as duas conexões seguiram pela rota A. Os resultados deste teste estão na tabela 5.7

Tabela 5.7: Roteamento IP na rede "peixe" pela rota A

\begin{tabular}{|l|l|l|l|l|c|c|}
\hline \%CPU & $\begin{array}{l}\text { \%CPU } \\
\text { LSR1 }\end{array}$ & $\begin{array}{l}\text { \%CPU } \\
\text { LSR2 }\end{array}$ & $\begin{array}{l}\text { \%SRU } \\
\text { LSR4 }\end{array}$ & $\begin{array}{l}\text { \%CPU } \\
\text { LSR5 }\end{array}$ & TCP1 (Mbps) & TCP2 (Mbps) \\
\hline 7 & 99 & - & - & 73 & 16,12 & 15,32 \\
\hline
\end{tabular}

Podemos perceber que LSR2 está em 99\%, e a soma dos tráfegos das duas conexões TCP é 31,44Mbps, que é um valor bem próximo ao valor da avaliação individual (figura 5.2). Isso indica que LSR2 está operando com sua capacidade máxima, limitando o tráfego nessa rede. LSR1 é um equipamento muito mais potente que os demais (vide figura 5.2), por isso a sua utilização de CPU é baixa $(7 \%)$

Após a execução dos testes com o netperf na rota A, a rede foi reconfigurada para utilizar a rota B (LSR1-LSR3-LSR4-LSR5, figura 5.14).

Para que o tráfego seguisse pela rota B, LSR1 passou a utilizar LSR3 (192.168.29.2) como roteador para a rede de HOSTB e LSR5 passou a utilizar LSR4 (192.168.29.65) como roteador para a rede de HOSTA:

lsr1: \# netstat $-r n$ 


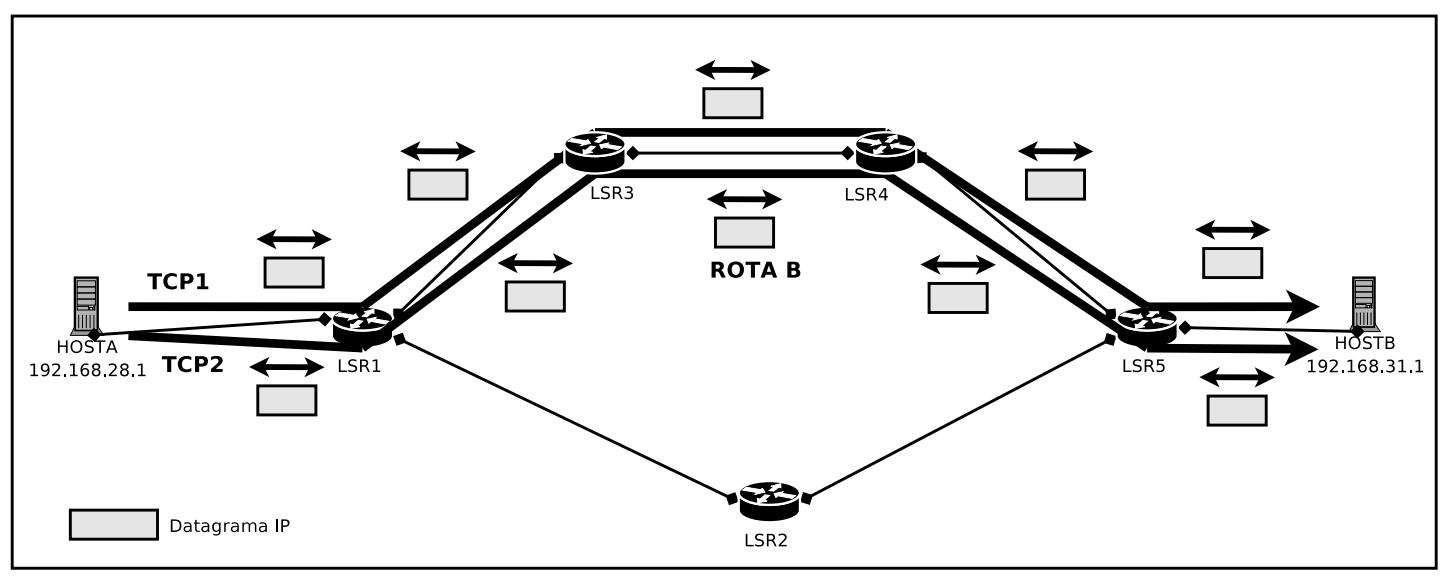

Figura 5.14: Roteamento IP: congestionamento na rota B

Kernel IP routing table

192.168 .28 .0

0.0 .0 .0

Genmask

Flags

MSS Window irtt Iface

192.168 .29 .0

0.0 .0 .0

$255.255 .255 .224 \mathrm{U}$

00

0 eth1

192.168 .30 .0

$0.0 \cdot 0.0$

$255.255 .255 .224 \mathrm{U}$

00

0 eth2

192.168 .31 .0

192.168.29. 2

$255.255 .255 .224 \mathrm{U}$

00

0 eth3

00

0 eth2

1sr5: * netstat -rn

Kernel IP routing table

Destination Gateway

192.168 .28 .0

192.168 .29 .65

Genmask

Flags

MSS Window irtt Iface

$192.168 .30 \cdot 32$

0.0 .0 .0

255.255 .255 .224 UG

00

0 eth1

192.168 .29 .64

0.0 .0 .0

$255.255 .255 .224 \mathrm{U}$

00

0 eth2

192.168 .31 .0

$0.0 \cdot 0.0$

$255.255 .255 .224 \mathrm{U}$

00

0 eth 1

$255.255 .255 .224 \mathrm{U}$

00

0 eth3

LSR3 e LSR4 fazem parte da rota B, portanto, precisam ser configurados para poder encaminhar os datagramas IP para seus respectivos destinos. LSR3 deve encaminhar datagramas para a rede de HOSTA através de LSR1 (192.168.29.1) e para a rede de HOSTB através de LSR4 (192.168.29.34), e LSR4 deve encaminhar datagramas para a rede de HOSTA através de LSR3 (192.168.29.33) e para a rede de HOSTB através de LSR5 (192.168.29.66):

1sr3: \# netstat $-r n$

Kernel IP routing table 


\begin{tabular}{|c|c|c|c|c|c|c|c|}
\hline Destination & Gateway & Genmask & Flags & MSS & Window & irtt & If ace \\
\hline 192.168 .28 .0 & 192.168 .29 .1 & 255.255 .255 .224 & UG & 0 & 0 & 0 & eth1 \\
\hline 192.168 .29 .0 & 0.0 .0 .0 & 255.255 .255 .224 & $\mathrm{U}$ & 0 & 0 & 0 & eth1 \\
\hline 192.168 .29 .32 & 0.0 .0 .0 & 255.255 .255 .224 & $\mathrm{U}$ & 0 & 0 & 0 & eth2 \\
\hline 192.168 .31 .0 & 192.168 .29 .34 & 255.255 .255 .224 & UG & 0 & 0 & 0 & eth2 \\
\hline $\begin{array}{l}\text { lsr4: \# netstat } \\
\text { Kernel IP routil }\end{array}$ & $\begin{array}{l}-r n \\
\text { ng table }\end{array}$ & & & & & & \\
\hline Destination & Gateway & Genmask & Flags & MSS & Window & irtt & If ace \\
\hline 192.168 .28 .0 & 192.168 .29 .33 & 255.255 .255 .224 & UG & 0 & 0 & 0 & eth1 \\
\hline 192.168 .30 .32 & 0.0 .0 .0 & 255.255 .255 .224 & $\mathrm{U}$ & 0 & 0 & 0 & eth1 \\
\hline 192.168 .29 .64 & 0.0 .0 .0 & 255.255 .255 .224 & $\mathrm{U}$ & 0 & 0 & 0 & eth2 \\
\hline 192.168 .31 .0 & 192.168 .29 .66 & 255.255 .255 .224 & UG & 0 & 0 & 0 & eth2 \\
\hline
\end{tabular}

Para confirmar a utilização da rota B, foram disparadas mensagens ICMP de HOSTA para HOSTB, e as interfaces de rede dos roteadores foram monitoradas com tcpdump. Desta vez, LSR2 é o roteador que não recebe tráfego.

Novamente, foram executados dois processos netperf de HOSTA em direção a HOSTB, e neste caso, todo o tráfego passou pela rota B. Os resultados deste teste estão na tabela 5.8 .

Tabela 5.8: Roteamento IP na rede "peixe" pela rota B

\begin{tabular}{|l|l|l|l|l|l|l|}
\hline $\begin{array}{l}\text { \%CPU } \\
\text { LSR1 }\end{array}$ & $\begin{array}{l}\text { \%CPU } \\
\text { LSR2 }\end{array}$ & $\begin{array}{l}\text { \%CPU } \\
\text { LSR3 }\end{array}$ & $\begin{array}{l}\text { \%CPU } \\
\text { LSR4 }\end{array}$ & $\begin{array}{l}\text { \%CPU } \\
\text { LSR5 }\end{array}$ & TCP1 & TCP2 \\
\hline 8 & - & 81 & 58 & 92 & 22,82 & 21,12 \\
\hline
\end{tabular}

Para o próximo teste nesta topologia, os roteadores foram configurados para encaminhar datagramas através de rótulos MPLS (figura 5.15).

Os LSRs foram configurados de maneira que cada conexão TCP siga por um LSP dedicado, aproveitando melhor recursos de rede que ficaram ociosos no roteamento IP. Este tipo de configuração utiliza informações da camada 4 (portas TCP) para diferenciar cada conexão e direcioná-las para o seu LSP.

O primeiro passo foi configurar os rótulos para os LSPs em direção a HOSTB. LSP1A é o caminho LSR1 -> LSR2 -> LSR5, e LSP1B é o caminho LSR1 -> 


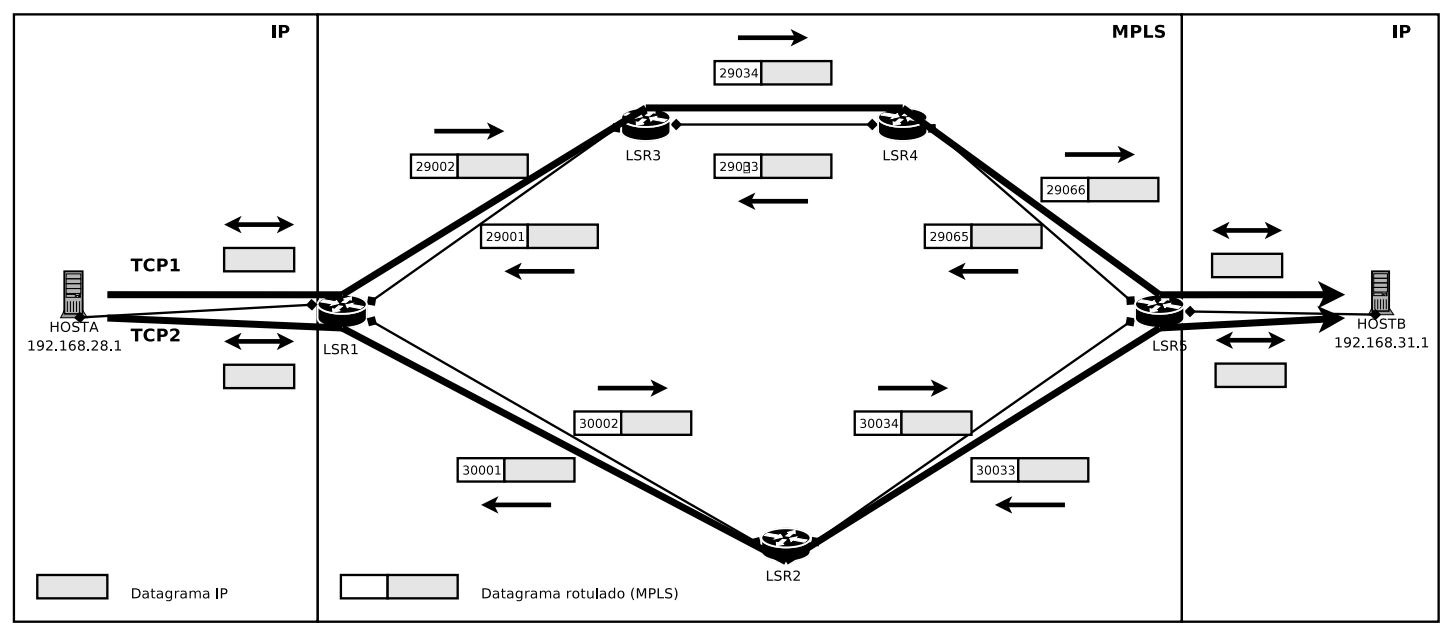

Figura 5.15: Roteamento IP/MPLS

LSR3 -> LSR4 -> LSR5.

LSR1 deve realizar as seguintes tarefas:

- Adicionar o rótulo 30002 para datagramas que devem seguir por LSP1A;

- Adicionar o rótulo 29002 para datagramas que devem seguir por LSP1B;

- Receber datagramas rotulados com o valor 29001 e encaminhá-los segundo informações do cabeçalho IP;

- Receber datagramas rotulados com o valor 30001 e encaminhá-los segundo informações do cabeçalho IP;

- O netperf utiliza uma conexão TCP na porta 12865 para realizar o controle do tráfego gerado, portanto, foi necessário direcionar esse tipo de tráfego para um dos LSPs;

- Direcionar o tráfego originado na rede 192.168.28.0/27 com destino à rede 192.168.31.0/27, porta 7000 (TCP1) para o LSP1A;

- Direcionar o tráfego originado na rede 192.168.28.0/27 com destino à rede 192.168.31.0/27, porta 7001 (TCP2) para o LSP1B.

Os rótulos de saída foram criados como nos testes anteriores, executando-se os seguintes comandos: 
lsr1: \# mpls nhlfe add key 0 instructions push gen 29002 ।

nexthop eth2 ipv4 192.168.29.2

Key: 0x00000002

lsr1: \# mpls nhlfe add key 0 instructions push gen 30002 \

nexthop eth3 ipv4 192.168.30.2

Key: 0x00000003

A configuração das interfaces de rede quanto ao labelspace e o mapeamento de rótulos de chegada também não trazem novidades em relação aos testes anteriores:

lsr1: \# mpls labelspace add dev eth2 labelspace 0

lsr1: \# mpls labelspace add dev eth3 labelspace 0

lsr1: \# mpls ilm add label gen 29001

lsr1: \# mpls ilm add label gen 30001

A primeira diferença neste teste está na configuração das rotas e no direcionamento de tráfego nos LERs.

Como o tráfego para o mesmo IP vai seguir simultaneamente por duas rotas (LSPs) diferentes, é preciso criar uma entrada na tabela de roteamento que possibilite a LSR1 utilizar dois nexthops: LSR2 (192.168.30.2) e LSR3 (192.168.29.2).

lsr1: \# ip route add 192.168.31.0/27 nexthop via 192.168.29.2\

nexthop via 192.168 .30 .2

O próximo passo é direcionar o tráfego para suas respectivas LSPs:

lsr1: \# iptables -A FORWARD -p icmp -s 192.168.28.0/27 -d 192.168.31.0/27 ।

--j spec_nh --spec_nh 0x8847:0x2

lsr1: \# iptables -A FORWARD -p tcp -s 192.168.28.0/27 -d 192.168.31.0/27 ।

--dport 12865 --j spec_nh --spec_nh 0x8847:0x2

lsr1: \# iptables -A FORWARD -p tcp -s 192.168.28.0/27 -d 192.168.31.0/27 ।

--dport 7000 --j spec_nh --spec_nh 0x8847:0x3

lsr1: \# iptables -A FORWARD -p tcp -s 192.168.28.0/27 -d 192.168.31.0/27 ।

--dport 7001 --j spec_nh --spec_nh 0x8847:0x2

Após a execução dos comandos anteriores, LSR1 ficou com a seguinte configuração: 
lsr1: \# mpls nhlfe show

NHLFE entry key 0x00000003 mtu 1496 propagate_ttl

push gen 30002 set eth3 ipv4 192.168.30.2 (0 bytes, 0 pkts, 0 dropped)

NHLFE entry key 0x00000002 mtu 1496 propagate_ttl

push gen 29002 set eth2 ipv4 192.168.29.2 (0 bytes, 0 pkts, 0 dropped)

lsr1: \# mpls ilm show

ILM entry label gen 30001 labelspace 0 proto ipv4

pop peek (0 bytes, 0 pkts, 0 dropped)

ILM entry label gen 29001 labelspace 0 proto ipv4

pop peek (0 bytes, 0 pkts, 0 dropped)

lsr1: \# mpls labelspace show

LABELSPACE entry dev eth0 labelspace -1

LABELSPACE entry dev lo labelspace -1

LABELSPACE entry dev eth1 labelspace -1

LABELSPACE entry dev eth2 labelspace 0

LABELSPACE entry dev eth3 labelspace 0

LABELSPACE entry dev tunl0 labelspace -1

1sr1: \# ip route list

$192.168 .31 .0 / 27$

nexthop via 192.168.29.2 dev eth2 weight 1

nexthop via 192.168.30.2 dev eth3 weight 1

192.168.29.0/27 dev eth2 proto kernel scope link src 192.168.29.1

192.168.30.0/27 dev eth3 proto kernel scope link src 192.168.30.1

192.168.28.0/27 dev eth1 proto kernel scope link src 192.168.28.2

143.107.180.0/24 dev etho proto kernel scope link src 143.107.180.173

default via $143.107 .180 .1 \mathrm{dev}$ eth0

1sr1: \# iptables -L -n

Chain INPUT (policy ACCEPT)

target prot opt source destination

Chain FORWARD (policy ACCEPT)

target prot opt source destination

spec_nh icmp - $\quad 192.168 .28 .0 / 27 \quad 192.168 .31 .0 / 27$

set spec_nh 0x4788:0x00000002

spec_nh tcp - $\quad 192.168 .28 .0 / 27 \quad 192.168 .31 .0 / 27$ 
tcp dpt:12865 set spec_nh 0x4788:0x00000002

spec_nh tcp - $\quad 192.168 .28 .0 / 27 \quad 192.168 .31 .0 / 27$

tcp dpt:7000 set spec_nh 0x4788:0x00000003

spec_nh tcp $--\quad 192.168 .28 .0 / 27 \quad 192.168 .31 .0 / 27$

tcp dpt:7001 set spec_nh 0x4788:0x00000002

Chain OUTPUT (policy ACCEPT)

target prot opt source destination

LSR5 tem uma função semelhante a de LSR1, e precisa da configuração de rótulos para os LSPs em direção a HOSTA. LSP2A é o caminho LSR5 -> LSR2 -> LSR1, e LSP2B é o caminho LSR5 -> LSR4 -> LSR3 -> LSR1. As funções de LSR5 são:

- Adicionar o rótulo 30033 para datagramas que devem seguir por LSP2A;

- Adicionar o rótulo 29065 para datagramas que devem seguir por LSP2B;

- Receber datagramas rotulados com o valor 29066 e encaminhá-los segundo informações do cabeçalho IP;

- Receber datagramas rotulados com o valor 30034 e encaminhá-los segundo informações do cabeçalho IP;

- Direcionamento da resposta da conexão de controle do netperf para um LSP. A principal característica de definição dessa FEC é a porta de origem no valor 12865 ;

- Direcionar o tráfego originado na rede 192.168.31.0/27 e porta 7000 (retorno de TCP1), com destino à rede 192.168.28.0/27 para o LSP2A;

- Direcionar o tráfego originado na rede 192.168.31.0/27 e porta 7001 (retorno de TCP2), com destino à rede 192.168.28.0/27, para o LSP2B. 
NHLFE entry key 0x00000003 mtu 1496 propagate_ttl

push gen 30033 set eth2 ipv4 192.168.30.33 (0 bytes, 0 pkts, 0 dropped)

NHLFE entry key 0x00000002 mtu 1496 propagate_ttl

push gen 29065 set eth1 ipv4 192.168.29.65 (0 bytes, 0 pkts, 0 dropped)

lsr5: \# mpls ilm show

ILM entry label gen 30034 labelspace 0 proto ipv4

pop peek (0 bytes, 0 pkts, 0 dropped)

ILM entry label gen 29066 labelspace 0 proto ipv4

pop peek ( 0 bytes, 0 pkts, 0 dropped)

lsr5: \# mpls labelspace show

LABELSPACE entry dev eth0 labelspace -1

LABELSPACE entry dev lo labelspace -1

LABELSPACE entry dev eth1 labelspace 0

LABELSPACE entry dev eth2 labelspace 0

LABELSPACE entry dev eth3 labelspace -1

lsr5: \# ip route list

$192.168 .28 .0 / 27$

nexthop via 192.168.29.65 dev eth1 weight 1

nexthop via 192.168 .30 .33 dev eth2 weight 1

192.168.29.64/27 dev eth1 proto kernel scope link src 192.168.29.66

192.168.30.32/27 dev eth2 proto kernel scope link src 192.168.30.34

192.168.31.0/27 dev eth3 proto kernel scope link src 192.168.31.2

143.107.180.0/24 dev etho proto kernel scope link src 143.107.180.179

default via $143.107 .180 .1 \mathrm{dev}$ eth0

1sr5: \# iptables -L $-\mathrm{n}$

Chain INPUT (policy ACCEPT)

target prot opt source destination

Chain FORWARD (policy ACCEPT)

target prot opt source destination

spec_nh icmp - $192.168 .31 .0 / 27 \quad 192.168 .28 .0 / 27$

set spec_nh 0x4788:0x00000002

spec_nh tcp - $\quad 192.168 .31 .0 / 27 \quad 192.168 .28 .0 / 27$

tcp spt:12865 set spec_nh 0x4788:0x00000002

spec_nh tcp $--\quad 192.168 .31 .0 / 27 \quad 192.168 .28 .0 / 27$ 
tcp spt:7000 set spec_nh 0x4788:0x00000003

spec_nh tcp - $\quad 192.168 .31 .0 / 27 \quad 192.168 .28 .0 / 27$

tcp spt:7001 set spec_nh 0x4788:0x00000002

Chain OUTPUT (policy ACCEPT)

target prot opt source destination

LSR2, LSR3 e LSR4 realizam apenas a troca de rótulos:

- Em LSP1A: LSR2 deve receber datagramas rotulados com o valor 30002 de LSR1, trocá-lo pelo valor 30034 e encaminhá-lo para LSR5 (192.168.30.34);

- Em LSP1B: LSR3 deve receber datagramas rotulados com o valor 29002 de LSR1, trocá-lo pelo valor 29034 e encaminhá-lo para LSR4 (192.168.29.34). LSR4 deve receber datagramas rotulados com o valor 29034 de LSR3, trocálo pelo valor 29066 e encaminhá-lo para LSR5 (192.168.29.66);

- Em LSP2A: LSR2 deve receber datagramas rotulados com o valor 30033 de LSR5, trocá-lo pelo valor 30001 e encaminhá-lo para LSR1 (192.168.30.1);

- Em LSP2B: LSR4 deve receber datagramas rotulados com o valor 29065 de LSR5, trocá-lo pelo valor 29033 e encaminhá-lo para LSR3 (192.168.29.33). LSR3 deve receber datagramas rotulados com o valor 29033 de LSR4, trocálo pelo valor 29001 e encaminhá-lo para LSR1 (192.168.29.1).

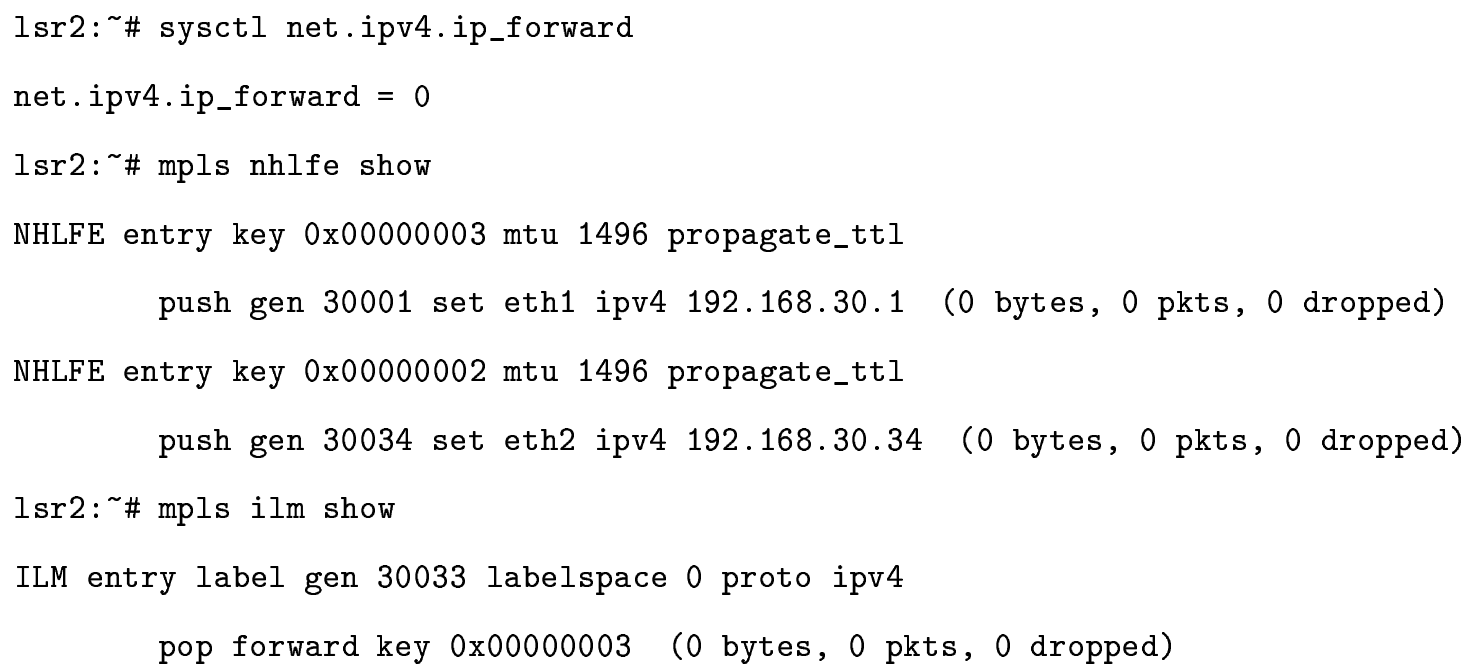


ILM entry label gen 30002 labelspace 0 proto ipv4 pop forward key 0x00000002 (0 bytes, 0 pkts, 0 dropped)

lsr3: \# sysctl net.ipv4.ip_forward

net.ipv4.ip_forward $=0$

lsr3: \# mpls nhlfe show

NHLFE entry key 0x00000003 mtu 1496 propagate_ttl

push gen 29001 set eth1 ipv4 192.168.29.1 (0 bytes, 0 pkts, 0 dropped)

NHLFE entry key 0x00000002 mtu 1496 propagate_ttl

push gen 29034 set eth2 ipv4 192.168.29.34 (0 bytes, 0 pkts, 0 dropped)

lsr3: \# mpls ilm show

ILM entry label gen 29033 labelspace 0 proto ipv4

pop forward key 0x00000003 (0 bytes, 0 pkts, 0 dropped)

ILM entry label gen 29002 labelspace 0 proto ipv4

pop forward key 0x00000002 (0 bytes, 0 pkts, 0 dropped)

lsr4: \# sysctl net.ipv4.ip_forward

net.ipv4.ip_forward $=0$

lsr4: \# mpls nhlfe show

NHLFE entry key 0x00000003 mtu 1496 propagate_ttl

push gen 29033 set eth1 ipv4 192.168.29.33 (0 bytes, 0 pkts, 0 dropped)

NHLFE entry key 0x00000002 mtu 1496 propagate_ttl

push gen 29066 set eth2 ipv4 192.168.29.66 (0 bytes, 0 pkts, 0 dropped)

lsr4: \# mpls ilm show

ILM entry label gen 29065 labelspace 0 proto ipv4

pop forward key 0x00000003 (0 bytes, 0 pkts, 0 dropped)

ILM entry label gen 29034 labelspace 0 proto ipv4

pop forward key 0x00000002 (0 bytes, 0 pkts, 0 dropped)

Com os LSPs configurados, novamente foram executados dois processos netperf de HOSTa para HOSTB. Os resultados obtidos na execução desse testes estão na tabela 5.9.

A figura 5.16 reúne os resultados dos três testes realizados nessa topologia (rede peixe), para facilitar a comparação de desempenho entre as arquiteturas.

Podemos observar dois fatos importantes: 
Tabela 5.9: Roteamento IP sobre MPLS na rede "peixe"

\begin{tabular}{|l|l|l|l|l|l|l|}
\hline \%CPU LSR1 & $\begin{array}{l}\text { \%CPU } \\
\text { LSR2 }\end{array}$ & $\begin{array}{l}\text { \%CPU } \\
\text { LSR3 }\end{array}$ & $\begin{array}{l}\text { \%CPU } \\
\text { LSR4 }\end{array}$ & $\begin{array}{l}\text { \%CPU } \\
\text { LSR5 }\end{array}$ & TCP1 & TCP2 \\
\hline 11 & 45 & 30 & 29 & 99 & 18,69 & 19,04 \\
\hline
\end{tabular}

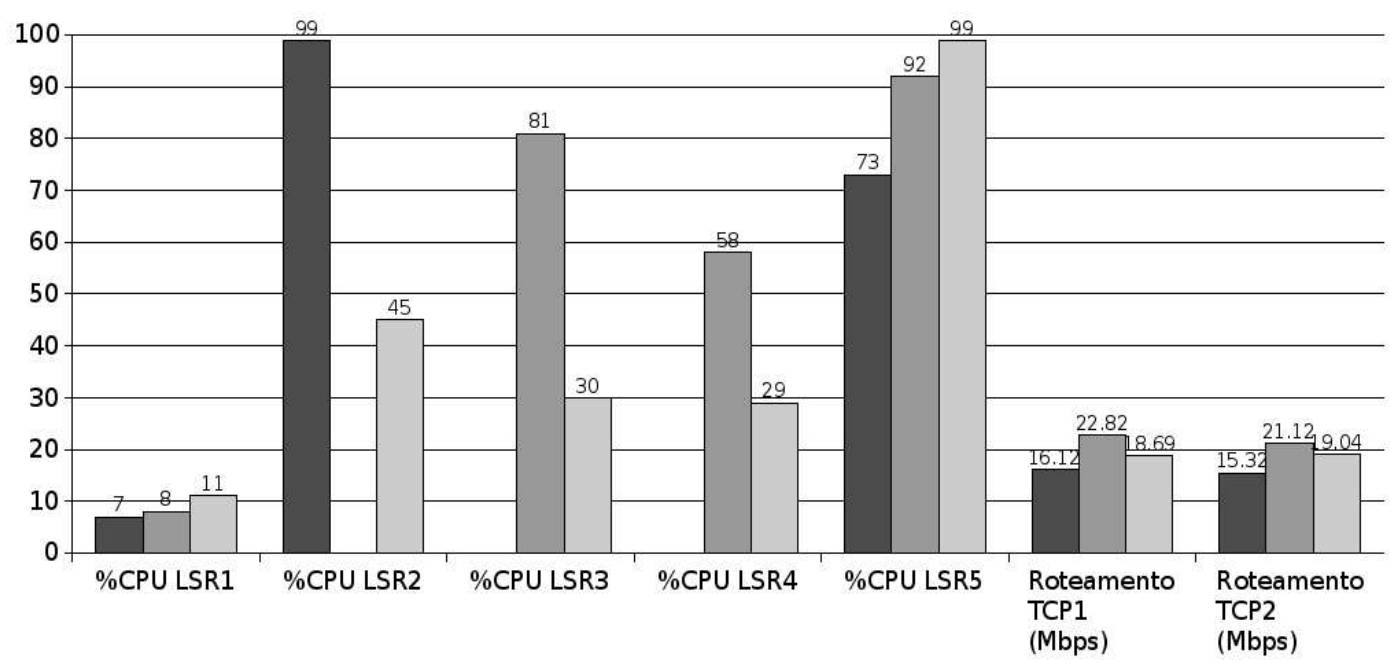

Roteamento IP (Rota A) $\square$ Roteamento IP (Rota B) $\square$ Roteamento IP sobre MPLS

Figura 5.16: Gráfico comparativo na "rede peixe"

- A rota B, mesmo tendo mais roteadores, tem maior desempenho que a rota A, porque esta tem o LSR de menor poder de processamento (LSR2);

- A utilização do protocolo MPLS não trouxe bons resultados, pois LSR5 não atende à demanda de processamento gerada pelas duas conexões TCP.

Para obtermos ganho de desempenho com a adição do MPLS, novamente é necessário reestruturar a rede, retirando os nós mais fracos das extremidades dos LSPs. LSR5 foi substituído por um roteador com maior poder de processamento (LSR6) e os testes foram executados novamente. Os resultados estão representados pelo gráfico da figura 5.17.

Após essa reestruturação, podemos perceber que:

- Mesmo com a substituição de LSR5 por LSR6, a rota A não teve ganho de desempenho no roteamento IP. Isso se deve ao fato de LSR2 continuar limitando o tráfego dessa rota; 


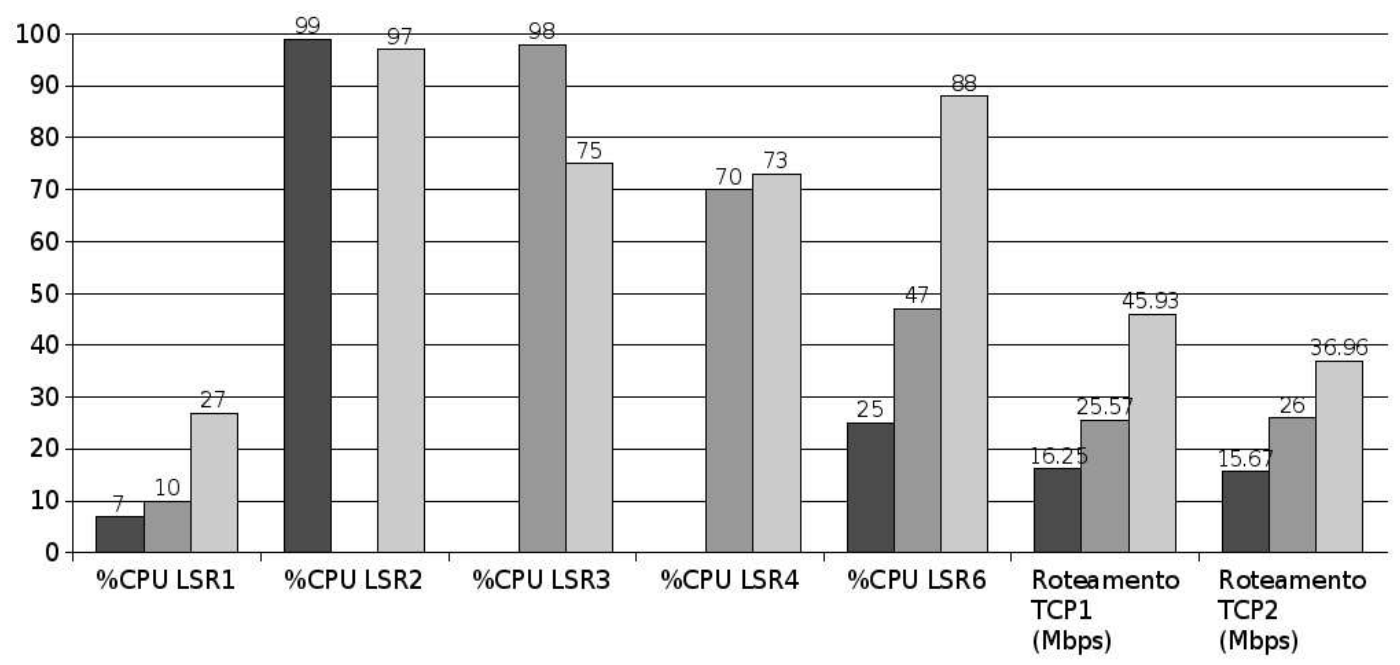

Roteamento IP (Rota A) $\square$ Roteamento IP (Rota B) $\square$ Roteamento IP sobre MPLS

Figura 5.17: Roteamento na rede "peixe" otimizada

- A rota B obteve ganho de desempenho no roteamento IP, pois LSR5 limitava o tráfego dessa rota. Após a substituição de LSR5 por LSR6, LSR3 passou a ser o fator limitante da rota B;

- O roteamento IP sobre MPLS proporcionou um ganho considerável de desempenho, pois a rota A foi utilizada para a conexão TCP2 e a rota B para a conexão TCP1. Essa configuração evita que uma das rotas fique ociosa e a outra sobrecarregada, caracterizando uma melhor utilização dos recursos de rede. 


\section{Capítulo 6}

\section{Conclusões}

A implementação do protocolo MPLS para Linux utilizada neste trabalho proporciona um ganho de desempenho em relação ao roteamento IP convencional em dois aspectos: desempenho e otimização de utilização dos recursos de rede.

\subsection{Desempenho}

As medições realizadas no capítulo anterior comprovam que o processo de encaminhamento apresenta um melhor desempenho com a utilização dos cabeçalhos MPLS, devido ao seu menor tamanho e complexidade. Isso possibilita o uso de equipamentos menos potentes em uma rede MPLS. Atualmente, já existem equipamentos que realizam o encaminhamento de datagramas IP em alta velocidade ("wire speed"), o que pode fazer com que o ganho de desempenho no encaminhamento de datagramas não seja mais motivo suficiente para a implementação do protocolo MPLS em redes com tais equipamentos.

A distribuição dos roteadores, conforme a sua capacidade de processamento, é muito importante na implementação de uma rede MPLS. Os roteadores de borda (LERs) são muito mais exigidos em uma rede MPLS, pois além de toda a análise que já era feita no roteamento IP convencional, os LERs precisam trabalhar na camada 2,5 . 
O uso da arquitetura IP sobre MPLS mostrou-se inapropriado em dois casos:

Redes com apenas dois roteadores: Como podemos observar no gráfico 6.1, uma rede com apenas dois roteadores não é um bom cenário para a utilização do protocolo MPLS, pois ambos os roteadores sofrem uma sobrecarga, e nenhum deles tem o benefício de processar apenas cabeçalhos MPLS;

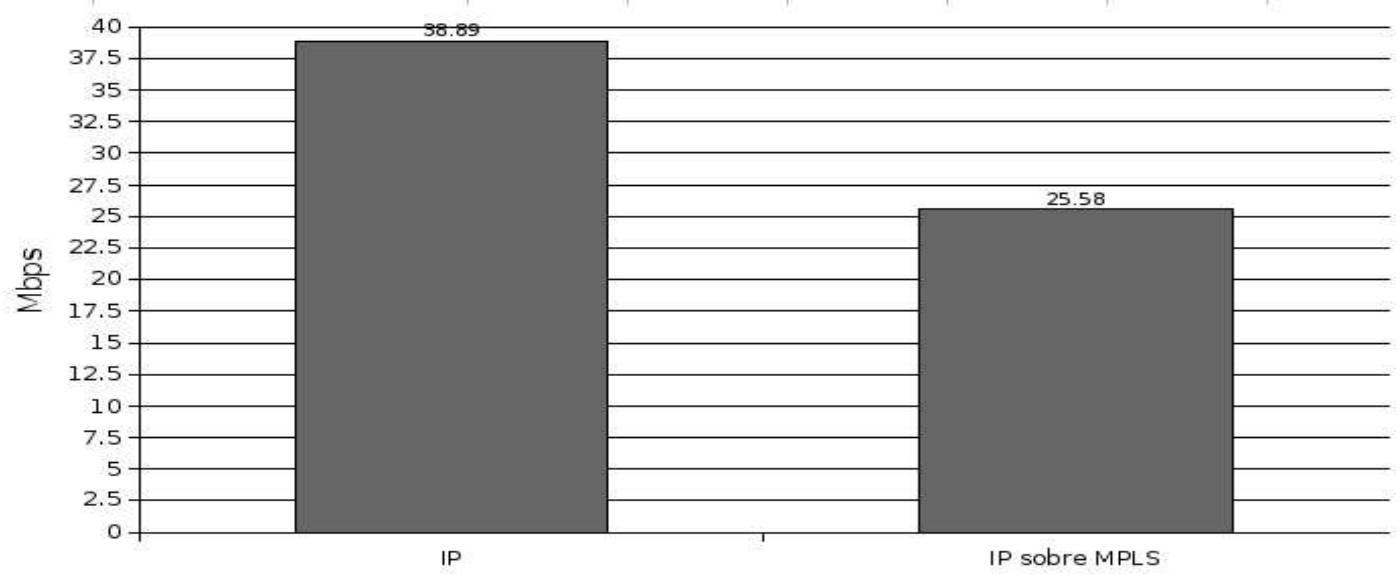

Figura 6.1: Desempenho com dois roteadores

Roteadores fracos nas bordas da rede MPLS: nesse caso, os roteadores de borda já estão limitando o tráfego em roteamento IP. Com a implementação do roteamento IP sobre MPLS, esses roteadores de borda tornam-se sobrecarregados, degradando ainda mais o desempenho da rede. Esse comportamento pode ser observado no gráfico 6.2 .

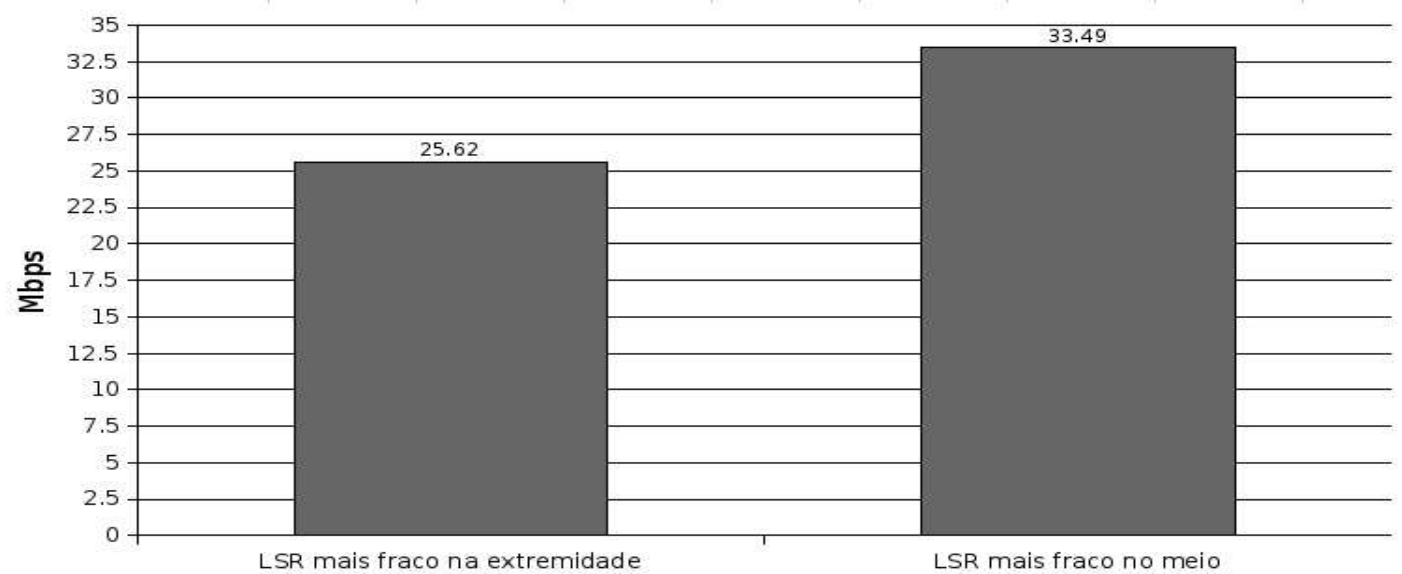

Figura 6.2: Posicionamento inadequado dos LSRs

Quando o equipamento mais fraco não esteve em uma das extremidades do 
LSP, a arquitetura IP sobre MPLS mostrou-se um pouco mais eficiente do que a arquitetura IP diretamente sobre Ethernet (figura 6.3).

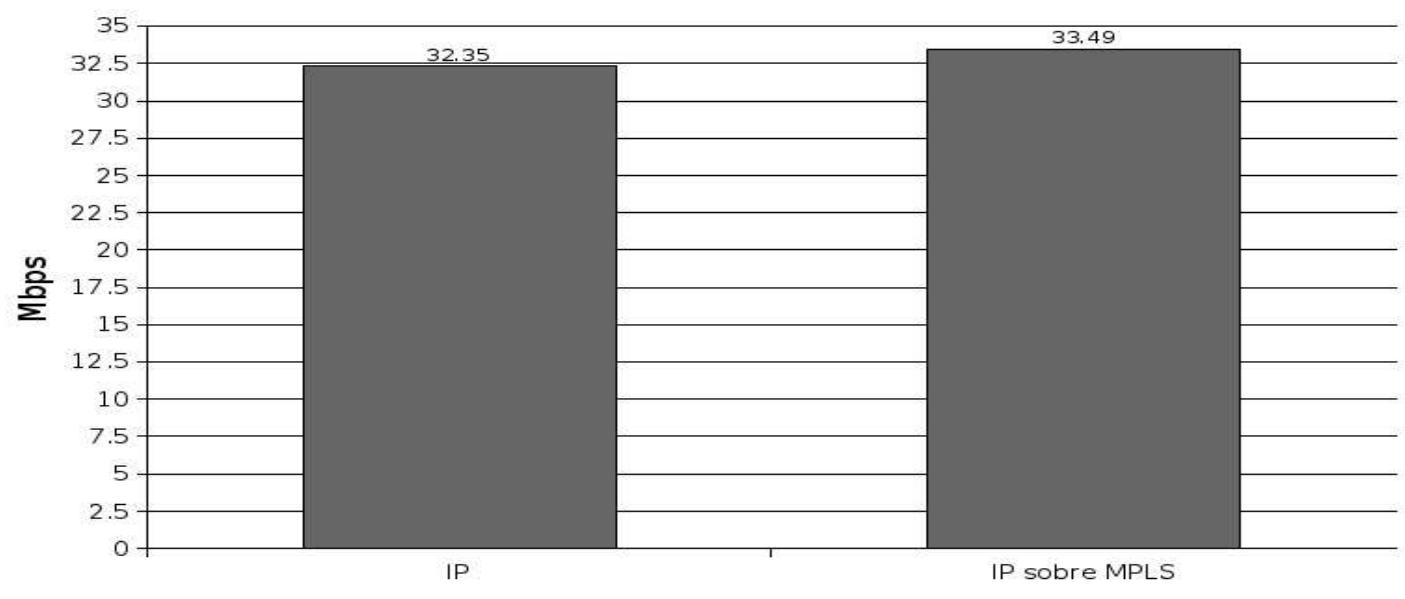

Figura 6.3: Posicionamento ideal dos LSRs

\subsection{Otimização de recursos}

O arquitetura IP sobre Ethernet fornece recursos pobres para implementação de engenharia de tráfego. A definição na origem da rota a ser seguida por um datagrama IP só pode ser feita através do campo IP Options, especificando-se todos os roteadores pelo qual esse datagrama deve passar. Essa técnica (source routing) tem duas desvantagens: é um processo custoso (exige análise do cabeçalho IP) e tem uma quantidade limitada de roteadores, devido ao tamanho máximo do cabeçalho IP.

Com o MPLS, a rota é definida no ingress LER, quando o datagrama IP recebe o cabeçalho MPLS com o valor de rótulo adequado. A partir desse LER, todos os LSRs do LSP fazem o encaminhamento utilizando a interface de rede e o LSR adequados (nexthops), forçando o datagrama rotulado a seguir pela rota (LSP) correta.

Os LSRs intermediários não possuem inteligência de roteamento e fazem apenas o encaminhamento de datagramas rotulados. Esse conceito contribui para a escalabilidade da rede, pois mesmo que o número de redes e roteadores aumente, 
todos os LSRs continuarão com o processo básico de encaminhamento e troca de rótulos.

Outro mecanismo oferecido pelo protocolo IP para qualidade de serviço são os serviços diferenciados (DiffServ), implementados através do campo TOS do cabeçalho IP [14]. Através do campo EXP do seu cabeçalho, o protocolo MPLS oferece um mapeamento das classes de serviço definidas pelo campo DSCP do cabeçalho IP, mantendo assim o comportamento adequado por parte dos LSRs nas políticas de descarte e prioridade de encaminhamento dos datagramas rotulados [37].

Finalmente, uma das características mais importantes do protocolo MPLS: a flexibilidade para a criação de LSPs. Enquanto que no roteamento IP a decisão de encaminhamento é feita apenas com base no endereço IP de destino, no roteamento IP sobre MPLS muitas informações podem ser utilizadas, inclusive informações de outras camadas do modelo OSI (portas TCP, interface Ethernet de entrada, entre outras). Nas medições realizadas neste trabalho, apenas a porta TCP foi utilizada para diferenciar o tráfego, mas qualquer informação que possa ser trabalhada com a ferramenta iptables poderia ter sido utilizada (no caso da implementação para Linux).

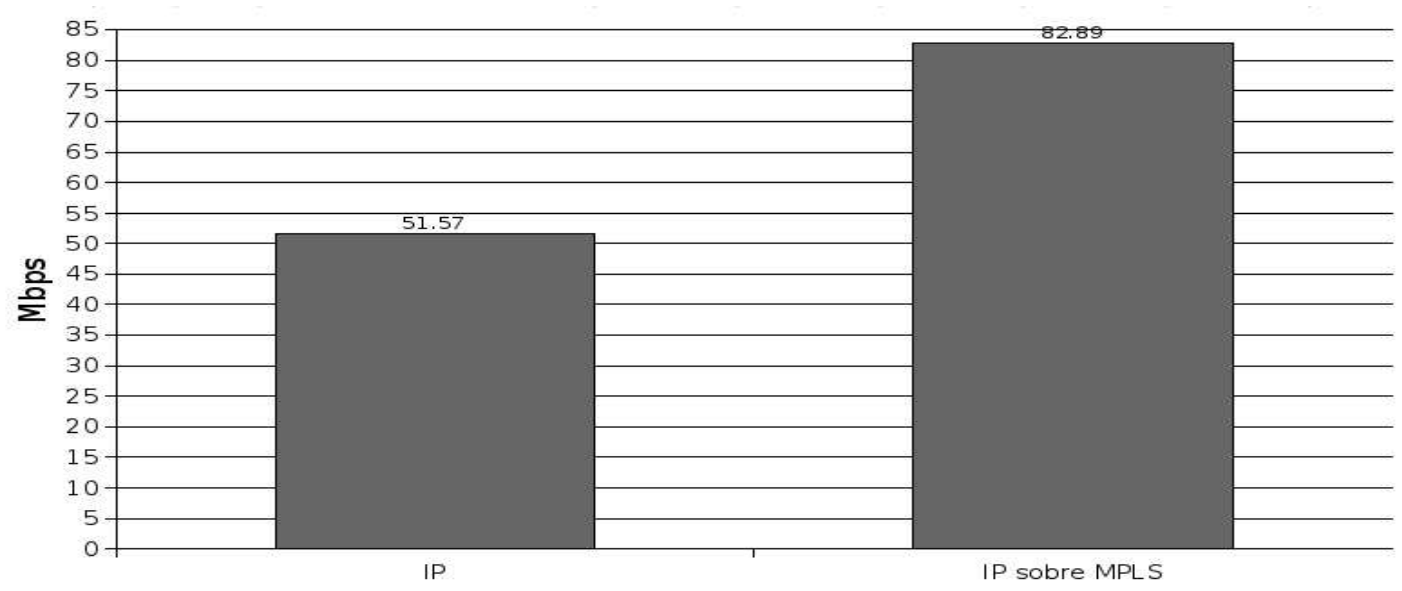

Figura 6.4: Segregação de tráfego

Segundo as figuras 5.17 e 6.4, a diferença entre o melhor caso no roteamento IP (rota B) e o roteamento IP sobre MPLS é de mais de 30Mbps, graças à separação 
de tráfego em menor granularidade (portas TCP) oferecida pela arquitetura IP sobre MPLS. Essa característica o mantém como um forte candidato para a implementação de engenharia de tráfego.

\subsection{Implementação: Projeto KyaTera}

Constatado o ganho de desempenho na arquitetura IP sobre MPLS, o próximo passo é a implementação dessa arquitetura em redes utilizadas no projeto KyaTera [45]. Nele estão sendo realizadas pesquisas de aplicações em grid [46] e [47], tráfego multimídia e simulações computacionais. Em São Carlos, quatro institutos/departamentos estão envolvidos nesse projeto: Instituto de Física de São Carlos (IFSC) [48], Departamento de Engenharia Elétrica - EESC (SEL-EESC), Instituto de Ciências Matemáticas e Computação (ICMC) e Departamento de Computação - UFSCAR (DC-UFSCAR). Todo o conhecimento e experiência adquiridos no desenvolvimento desta dissertação serão utilizados na implementação da rede para o KyaTera em São Carlos. A figura 6.3 ilustra o protótipo da rede construída para as avaliações iniciais da arquitetura IP sobre MPLS. 


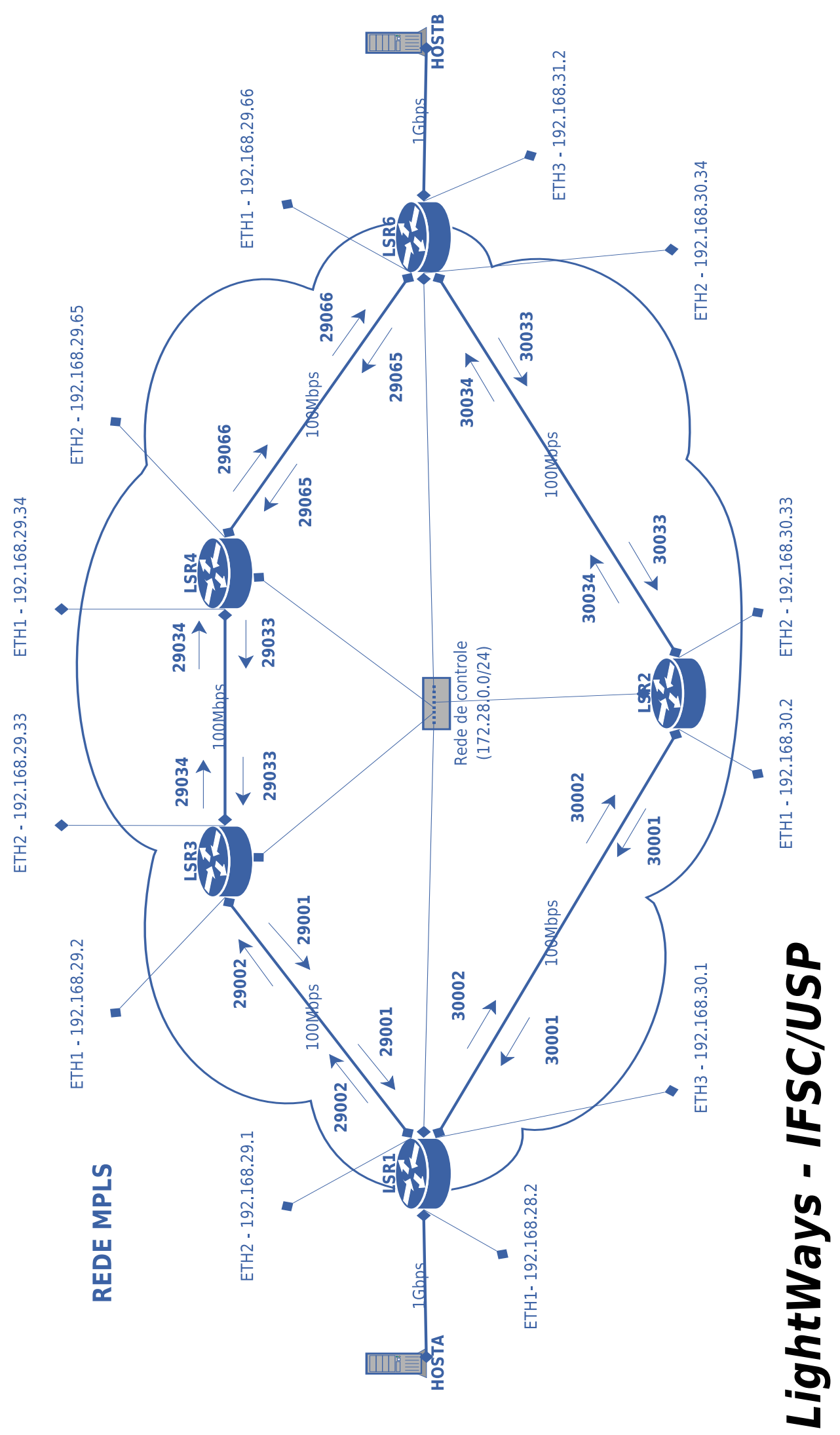

Figura 6.5: Protótipo para o KyaTera 


\subsection{Considerações finais}

Pelos resultados obtidos neste trabalho, podemos concluir que a arquitetura IP sobre MPLS é uma alternativa viável de sistema de roteamento. O formato do cabeçalho MPLS (menor e menos complexo que o cabeçalho IP) faz com que os LSRs sejam menos exigidos no armazenamento e processsamento das tabelas de encaminhamento. Outro benefício do protocolo MPLS é a utilização de um único tipo de algoritmo de busca na tabela de rotas (coincidência exata), o que não ocorre com o protocolo IP. Estes pontos positivos favorecem a utilização de equipamentos menos potentes como LSRs.

Os recursos oferecidos pela arquitetura IP sobre MPLS para a engenharia de tráfego são amplos, dada a granularidade fina que pode ser obtida no ingress LER. Qualquer informação que o ingress LER seja capaz de extrair de um datagrama IP pode ser utilizada como referência para segregar o tráfego de rede e criar os LSPs. 


\section{Capítulo 7}

\section{Trabalhos futuros}

O foco deste trabalho foi analisar e comparar o desempenho do processo de encaminhamento/roteamento e otimização de recursos oferecidos pelas arquiteturas IP e IP sobre MPLS em redes Ethernet. Em [49] pode ser encontrado um ótimo conjunto de experimentos com o protocolo MPLS.

Algumas abordagens interessantes para dar continuidade a este trabalho seriam:

- Estudo do desempenho do MPLS em outras tecnologias de nível 2 (ATM, PPP, Frame Relay), pois este trabalho analisou apenas redes Ethernet;

- Estudo dos protocolos de comunicação entre os LSRs (LDP [34] e RSVP [22]): assim como em redes IP, a manutenção de redes MPLS exige dinamismo e respostas rápidas em situações de falha dos equipamentos. A administração manual é muito lenta e propensa a erros, e piora com o aumento da quantidade de roteadores de um domínio. O protocolo MPLS permite o uso de diferentes protocolos de comunicação;

- Estudo do suporte a DiffServ: o protocolo MPLS oferece suporte aos serviços diferenciados através do campo EXP do seu cabeçalho;

- Avaliação de desempenho da técnica Penultimate Hop Popping [29]: essa técnica alivia os LERs em uma operação na pilha MPLS, transferindo essa 
função para o penúltimo LSR. Em topologias em que o core possui equipamentos mais potentes que as bordas, a técnica penultimate hop popping pode oferecer mais um ganho de desempenho;

- Estudo de Virtual Private Network (VPN) [50] com MPLS [51]: como o MPLS faz o encapsulamento do datagrama IP, ele possibilita a criação de VPNs;

- Análise (código) da implementação em Linux: mesmo havendo ganho de desempenho com a utilização do MPLS em roteadores Linux, seria interessante uma análise do seu código para se avaliar a influência da implementação no desempenho do encaminhamento/roteamento nos LSRs. Além disso, as especificações do protocolo MPLS [29] e [35] oferecem mais de uma alternativa no comportamento dos LSRs em algumas situações;

- Avaliação e comparação de outras implementações: por ser uma implementação gratuita General Public License (GPL) e utilizar computadores de baixo custo, a solução MPLS em Linux é muito atraente. Seria interessante um estudo comparativo com as implementações proprietárias, principalmente as implementações em hardware. Implementações em hardware geralmente têm melhor desempenho do que as implementações em software;

- Porte para FreeBSD [52]: como projeto pessoal do autor desta dissertação, com o objetivo de adquirir conhecimento aprofundado em desenvolvimento para sistemas operacionais (ferramentas, metodologias, arquitetura e implementação de protocolos de rede). 


\section{Referências Bibliográficas}

[1] Leiner, B. M.; et al. A Brief History of the Internet. http://www.isoc. org/internet/history/brief . shtml, último acesso em 09/08/2006.

[2] Kleinrock, L. Information Flow in Large Communication Nets. http: //www . lk.cs.ucla.edu/full_bibliography.html, último acesso em 09/08/2006.

[3] Tanenbaum, A. S. Computer Networks. 4th ed. Prentice Hall, 2003.

[4] Cisco Systems. X.25 Overview. http://www.cisco.com/univercd/cc/td/ doc/cisintwk/ito_doc/x25.htm, último acesso em 23/10/2006.

[5] Mogul, J.; Deering, S. RFC 1191: Path MTU Discovery. Network Working Group, 1990.

[6] McCann, J.; Deering, S.; Mogul, J. RFC 1981: Path MTU Discovery for IP version 6. Network Working Group, 1996.

[7] Internet Assigned Numbers Authority. RFC 3330: Special-Use IPv4 Addresses. The Internet Society, 2002.

[8] Deering, S.; Hinden, R. RFC 2460: Internet Protocol, Version 6 (IPv6) Specification. The Internet Society, 1998.

[9] Internet Systems Consortium Inc. Internet Systems Consortium. http: //www.isc.org/, último acesso em 09/08/2006.

[10] Rekhter, Y.; Moskowitz, B.; Karrenberg, D.; Groot, G. J.; Lear, E. RFC 1918: Address Allocation for Private Internets. Network Working Group, 1996.

[11] Srisuresh, P.; Egevang, K. RFC 3022: Traditional IP Network Address Translator (Traditional NAT). The Internet Society, 2001.

[12] Information Sciences Institute. RFC 791: Internet Protocol. DARPA, 1981.

[13] Almquist, P. RFC 1349: Type of Service in the Internet Protocol Suite. Network Working Group, 1992.

[14] Nichols, K.; Blake, S.; Baker, F.; Black, D. RFC 2474: Definition of the Differentiated Services Field (DS Field) in the IPv4 and IPv6 Headers. The Internet Society, 1998. 
[15] Ramakrishnan, K.; Floyd, S.; Black, D. RFC 3168: The Addition of Explicit Congestion Notification (ECN) to IP. The Internet Society, 2001.

[16] Baker, F. RFC 1812: Requirements for IP Version 4 Routers. Network Working Group, 1995.

[17] Comer, D. E. Internetworking with TCP/IP. 4th ed. Prentice Hall, 2000.

[18] Halabi, B. Internet Routing Architectures. New Riders Pub, 1997.

[19] Rede Nacional de Ensino e Pesquisa. O Protocolo BGP-4. http: //www.rnp. $\mathrm{br} /$ newsgen/9903/bgp4.html, último acesso em 23/10/2006.

[20] Moy, J. RFC 2178: OSPF Version 2. Network Working Group, 1998.

[21] Fonseca, J. L. A.; Stanton, M. A. Estudo experimental de videoconferência pessoal em inter-redes IP com QoS. http://www.rnp.br/newsgen/0111/ j1_wtr.html, último acesso em 09/08/2006.

[22] Braden, R.; Zhang, L.; Berson, S.; Herzog, S.; Jamin, S. RFC 2205: Resource ReSerVation Protocol (RSVP) - Version 1 Functional Specification. Network Working Group, 1997.

[23] Awduche, D.; Chiu, A.; Elwalid, A.; Widjaja, I.; Xiao, X. RFC 3272: Overview and Principles of Internet Traffic Engineering. The Internet Society, 2002 .

[24] Blake, S.; Black, D.; Carlson, M.; Davies, E.; Wang, Z.; Weiss, W. RFC 2475: An Architecture for Differentiated Services. The Internet Society, 1998.

[25] Braden, R.; Clark, D.; Shenker, S. RFC 1633: Integrated Services in the Internet Architecture: an Overview. Network Working Group, 1994.

[26] Davie, B.; et al. RFC 3246: An Expedited Forwarding PHB (Per-Hop Behavior). The Internet Society, 2002.

[27] Heinanen, J.; Baker, F.; Weiss, W.; Wroclawski, J. RFC 2597: Assured Forwarding PHB Group. The Internet Society, 1999.

[28] Postel, J. RFC 792: Internet Control Message Protocol. DARPA, 1981.

[29] Rosen, E.; Viswanathan, A.; Callon, R. RFC 3031: Multiprotocol Label Switching Architecture. The Internet Society, 2001.

[30] Newman, P.; Edwards, W. L.; Hinden, R.; Hoffman, E.; Ching Liaw, F.; Lyon, T.; Minshall, G. RFC 1953: Ipsilon Flow Management Protocol Specification for IPv4 Version 1.0. Network Working Group, 1996.

[31] Rekhter, Y.; Davie, B.; Katz, D.; Rosen, E.; Swallow, G. RFC 2105: Cisco Systems' Tag Switching Architecture Overview. Network Working Group, 1997. 
[32] Davie, B.; Rekhter, Y. MPLS Technology and Applications. Morgan Kaufmann, 2000.

[33] Harnedy, S. The MPLS Primer. Prentice Hall, 2002.

[34] Anderson, L.; Doolan, P.; Feldman, N.; Fredette, A.; Thomas, B. RFC 3036: LDP Specification. The Internet Society, 2001.

[35] Rosen, E.; Tappan, D.; Fedorkow, G.; Rekhter, Y.; Farinacci, D.; Li, T.; Conta, A. RFC 3032: MPLS Label Stack Encoding. The Internet Society, 2001.

[36] Davie, B.; Lawrence, J.; McCloghrie, K.; Rosen, E.; Swallow, G.; Rekhter, Y.; Doolan, P. RFC 3035: MPLS using LDP and ATM VC Switching. The Internet Society, 2001.

[37] Le Faucheur, F.; Wu, L.; Davie, B.; Davari, S.; Vaananen, P.; Krishnan, R.; Cheval, P.; Heinanen, J. RFC 3270: Multi-Protocol Label Switching (MPLS) Support of Differentiated Services. The Internet Society, 2002.

[38] Ooms, D.; Sales, B.; Livens, W.; Acharya, A.; Griffoul, F.; Ansari, F. RFC 3353: Overview of IP Multicast in a Multi-Protocol Label Switching (MPLS) Environment. The Internet Society, 2002.

[39] Awduche, D.; Malcolm, J.; Agogbua, J.; O'Dell, M.; McManus, J. RFC 2702: Requirements for Traffic Engineering Over MPLS. The Internet Society, 1999.

[40] Bradner, S.; McQuaid, J. RFC 2544: Benchmarking Methodology for Network Interconnect Devices. Network Working Group, 1999.

[41] Jones, R. NETPERF. http://www . netperf .org/netperf/NetperfPage. html, último acesso em 09/08/2006.

[42] Linux Online, Inc. The Linux Home Page at Linux Online. http://www. linux.org/, último acesso em 14/08/2006.

[43] Leu, J. R. MPLS for Linux. http://mpls-linux.sourceforge.net/, último acesso em 09/08/2006.

[44] Netfilter Core Team. Netfilter. http://www.netfilter.org/, último acesso em 09/08/2006.

[45] FAPESP. KyaTera. http://www.kyatera.fapesp.br/, último acesso em 09/08/2006.

[46] The Globus Alliance. The Globus Alliance. http://www.globus.org/, último acesso em 09/08/2006.

[47] Global GridForum. Global Grid Forum. http://www.gridforum.org/, último acesso em 09/08/2006. 
[48] Instituto de Física de São Carlos. IFSC - USP - Instituto de Física de São Carlos. http://www.ifsc.usp.br/, último acesso em 09/08/2006.

[49] Popa, A. MPLS-Linux labs. http://www.elcom.pub.ro/〜adrian.popa/ mpls-linux/mpls-linux-docs/, último acesso em 25/10/2006.

[50] Virtual Private Network Consortium. VPN Protocols. http://www.vpnc. org/vpn-standards.html, último acesso em 09/08/2006.

[51] Muthukrishnan, K.; Malis, A. RFC 2917: A Core MPLS IP VPN Architecture. The Internet Society, 2000.

[52] The FreeBSD Project. The FreeBSD Project. http://www.freebsd.org/, último acesso em 14/08/2006. 